\title{
PIV Uncertainty Methodologies for CFD Code Validation at the MIR Facility
}

Piyush Sabharwall

Thomas Conder

Richard Skifton

Carl Stoots

Eung Soo Kim

December 2013

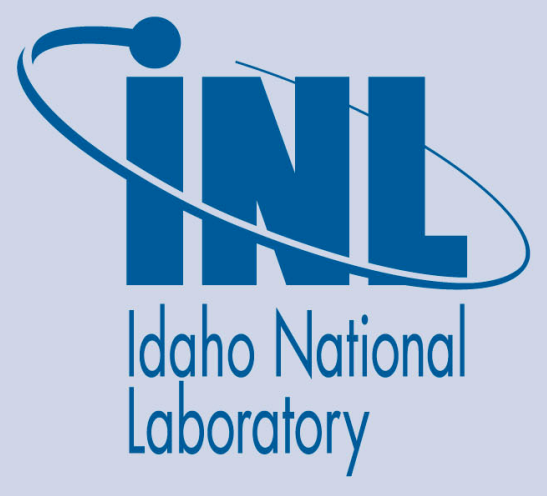

The INL is a U.S. Department of Energy National Laboratory operated by Battelle Energy Alliance 


\section{DISCLAIMER}

This information was prepared as an account of work sponsored by an agency of the U.S. Government. Neither the U.S. Government nor any agency thereof, nor any of their employees, makes any warranty, expressed or implied, or assumes any legal liability or responsibility for the accuracy, completeness, or usefulness, of any information, apparatus, product, or process disclosed, or represents that its use would not infringe privately owned rights. References herein to any specific commercial product, process, or service by trade name, trade mark, manufacturer, or otherwise, does not necessarily constitute or imply its endorsement, recommendation, or favoring by the U.S. Government or any agency thereof. The views and opinions of authors expressed herein do not necessarily state or reflect those of the U.S. Government or any agency thereof. 
INL/EXT-12-27728

\section{PIV Uncertainty Methodologies for CFD Code Validation at the MIR Facility}

Piyush Sabharwall, Thomas Conder, Richard Skifton, and Carl Stoots (Idaho National Laboratory);

Eung Soo Kim (Seoul National University)

December 2013

Idaho National Laboratory Idaho Falls, Idaho 83415

http://www.inl.gov

Prepared for the

U.S. Department of Energy

Office of Nuclear Energy

Under DOE Idaho Operations Office

Contract DE-AC07-05ID14517 



\section{ACKNOWLEDGEMENTS}

The authors would like to thank the Idaho National Laboratory

laboratory-directed research and development, which provided funding for this work. The authors greatly appreciate the effort of the research teams at Utah State University led by Prof. Barton Smith and the research team at Virginia Technical University led by Prof. Pavlos Vlachos, and for the contributing section (see Appendix B), Development of an Uncertainty Quantification Method for Matched Index of Refraction Facility. 


\section{EXECUTIVE SUMMARY}

Currently, computational fluid dynamics (CFD) is widely used in the nuclear thermal-hydraulics field for design and safety analyses. To validate CFD codes, high-quality multi-dimensional flow field data are essential. The Matched Index of Refraction (MIR) Flow Facility at Idaho National Laboratory has a unique capability to contribute to the development of validated CFD codes through the use of Particle Image Velocimetry (PIV). The significance of the MIR facility is that it permits non-intrusive velocity measurement techniques, such as PIV, through complex models without requiring probes and other instrumentation that disturb the flow. At the heart of any PIV calculation is the cross-correlation, which is used to estimate the displacement of particles in some small part of the image over the time span between two images. This image displacement is indicated by the location of the largest peak. In the MIR facility, uncertainty quantification is a challenging task due to the use of optical measurement techniques. Currently, this study is developing a reliable method to analyze uncertainty and sensitivity of the measured data and develop a computer code to automatically analyze the uncertainty/sensitivity of the measured data. The main objective of this study is to develop a well-established uncertainty quantification method for the MIR Flow Facility, which consists of many complicated uncertainty factors. In this study, the uncertainty sources are resolved in depth by categorizing them into uncertainties from the MIR flow loop and PIV system (including particle motion, image distortion, and data processing). Then, each uncertainty source is mathematically modeled or adequately defined. Finally, this study will provide a method and procedure to quantify the experimental uncertainty in the MIR Flow Facility with sample test results.

MIR results are in the form of three-component (3C) time average velocities and Reynolds stresses. The primary focus of this Laboratory Directed Research and Development is to provide uncertainties on these quantities.

Two independent methods for computation of uncertainty from two-component PIV measurements have been developed and are discussed. These are called the Uncertainty Surface Method and the Signal to Noise Ratio method.

In the Uncertainty Surface Method, an algorithm is tested to determine the algorithm's response to various uncertainty contributors, and the result of these tests (the uncertainty surface) is used to determine the uncertainty of each vector. The Signal to Noise Ratio method is based on the hypothesis that the image cross-correlation on which PIV is based contains information on the uncertainty, and that the magnitude of the correlation peak, if quantified properly, is all one needs to know to determine the uncertainty.

In each case, the uncertainty of every vector (in space and time) is determined. This provides the fundamental information for the $3 \mathrm{C}$ uncertainty. This information is propagated to $3 \mathrm{C}$ uncertainty using the same stereo calibration information that is used to compute $3 \mathrm{C}$ velocities from twocomponent vector fields. Once instantaneous $3 \mathrm{C}$ uncertainties are found for all space and time, these are propagated into the time-averaged velocities and Reynolds stresses using methods developed in this Laboratory Directed Research and Development.

The uncertainty quantification method developed in this study will lead to high-quality MIR data with accurate and reliable uncertainty quantification, and it will be a tremendous asset to the ongoing efforts to validate advanced computational methods. The MIR facility has tremendous potential of becoming a user facility for basic and applied research by government, industry, and academia where accurate and reliable uncertainty quantification is essential for producing high-quality data for computer code validation. The benefit to Idaho National Laboratory will derive directly from the ability of the MIR Lab to produce reliable estimates of uncertainty for the velocity field data collected with the PIV system. PIV uncertainty is critical to the validation of CFD codes, but currently, no practical and accepted method exists for estimating the uncertainty in PIV data, as mentioned earlier. 


\section{CONTENTS}

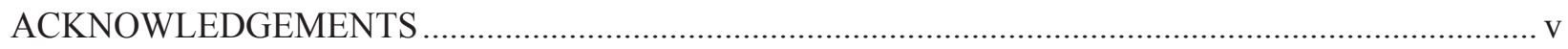

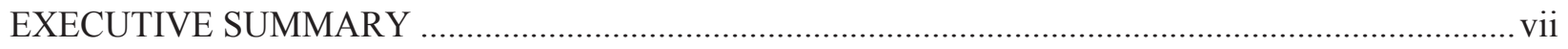

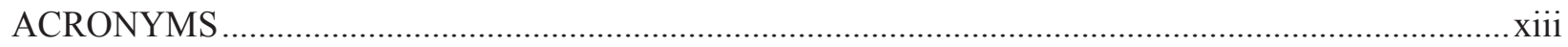

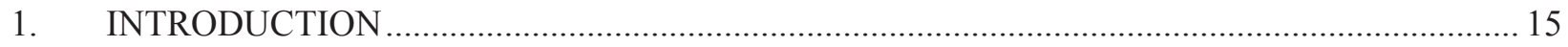

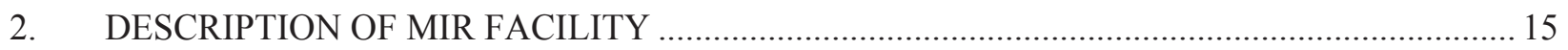

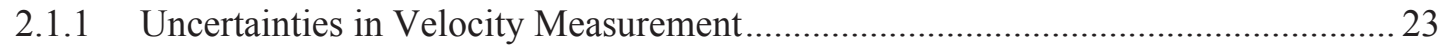

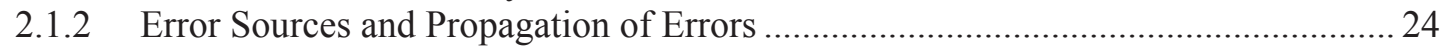

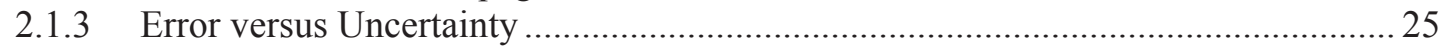

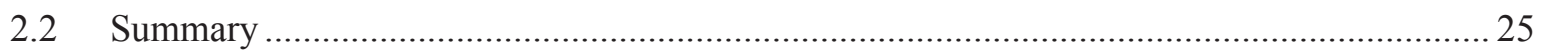

3. EFFECT OF OIL TEMPERATURE ON PIV MEASUREMENT ERROR IN THE MIR

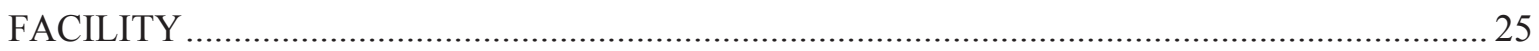

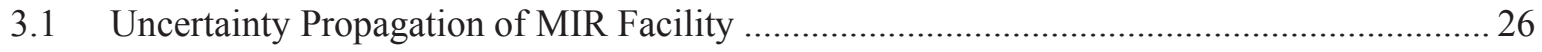

3.2 Estimation Method of Velocity Error by Mineral Oil Temperature .................................... 27

3.3 Analyses Method (Global Sensitivity Analyses) .............................................................29

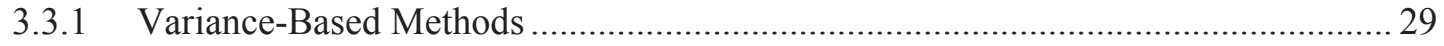

3.3.2 General Analysis Procedure for Global Sensitivity Analyses and SimLab .............. 30

3.3.3 General Strength and Weakness of Global Sensitivity Analyses............................... 31

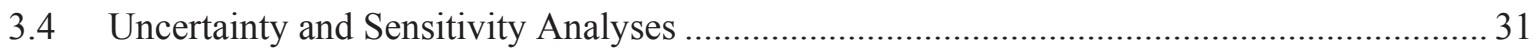

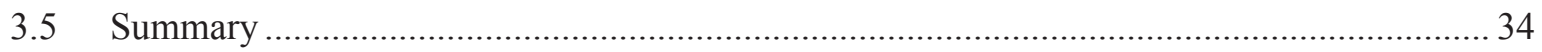

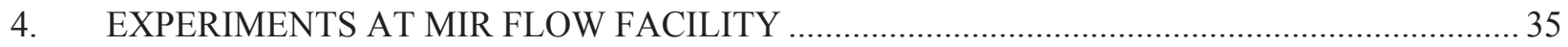

4.1 Bypass Flow Measurements in a Representative Gas-Cooled Prismatic Reactor Core

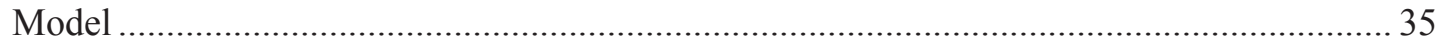

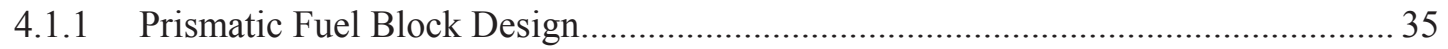

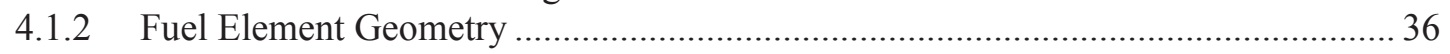

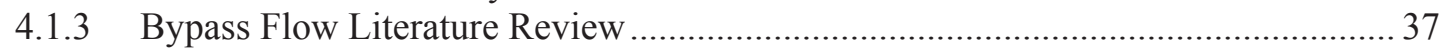

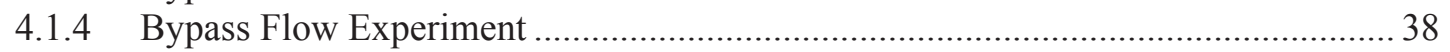

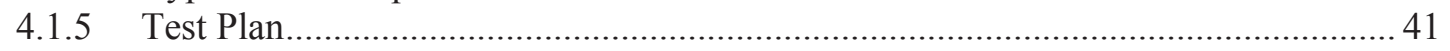

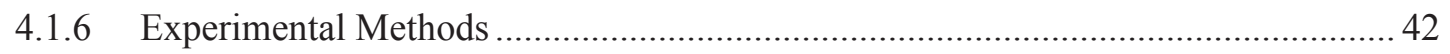

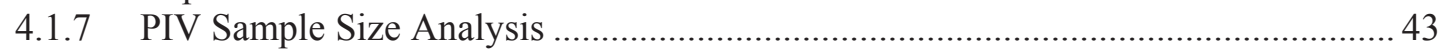

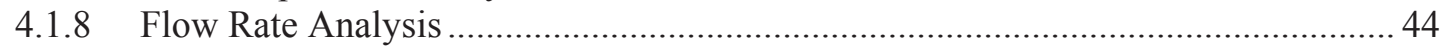

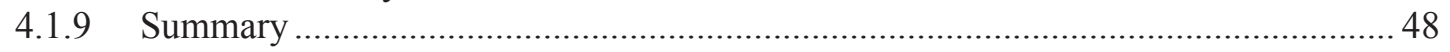

4.2 Bypass Transitional Flow Measurements of a Flat Plate .................................................. 48

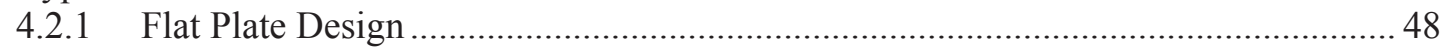

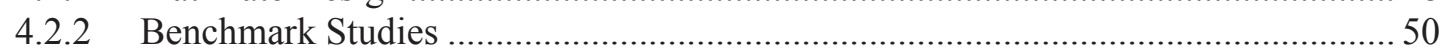

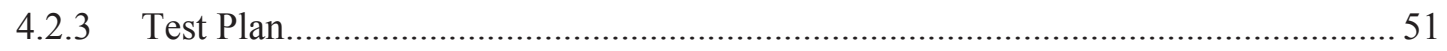

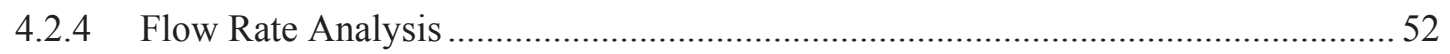

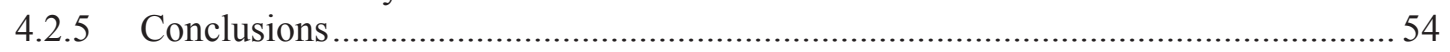

5. DEVELOPING AN UNCERTAINTY QUANTIFICATION METHOD FOR THE MIR

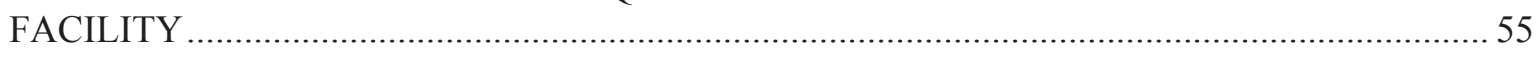

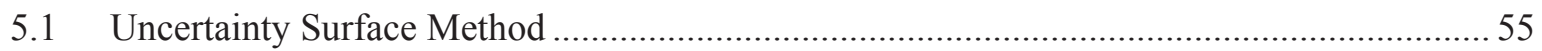




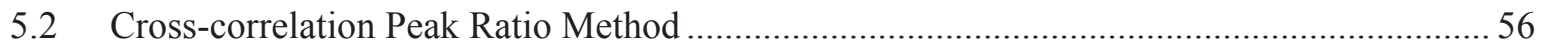

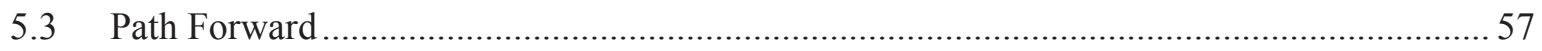

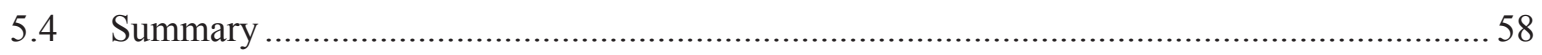

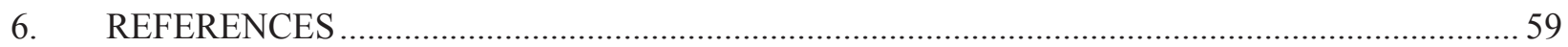

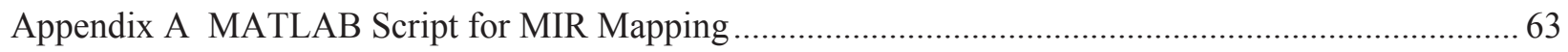

Appendix B Uncertainty Quantification Methodology For MIR Facility .............................................. 69

Appendix B-1 Creating a New Uncertainty Surface .................................................................... 99

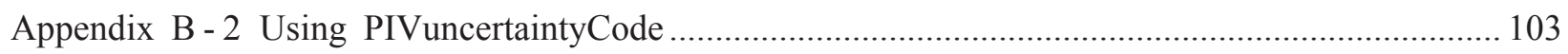

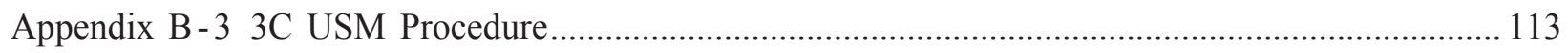

\section{FIGURES}

Figure 1. Index matching of quartz and mineral oil — part (a) demonstrates the quartz outside the oil and (b) demonstrates the quartz while submersed in the oil................................................ 16

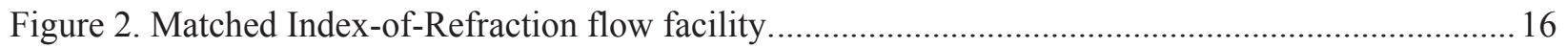

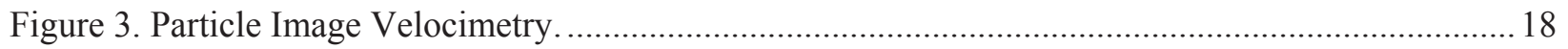

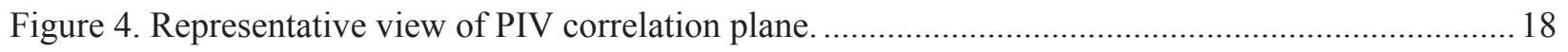

Figure 5. Identified uncertainty propagation structure and sources in MIR facility................................25

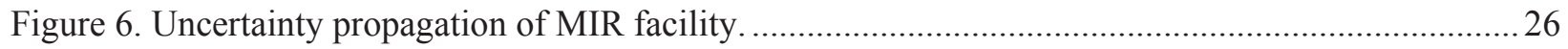

Figure 7. Basic idea of estimating velocity error from deviated indices of refractions...........................28

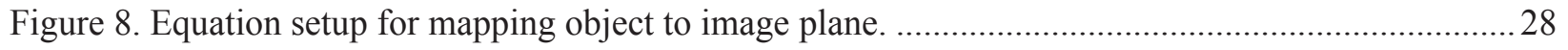

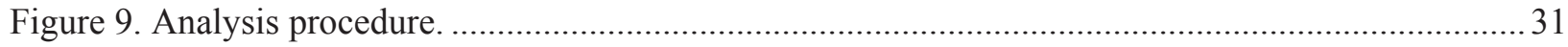

Figure 10. Estimated velocity measurement error from oil temperature ................................................ 32

Figure 11. Effect of temperature uncertainty on velocity measurement error. ......................................... 33

Figure 12. First order sensitivity index (Main Effect). ......................................................................... 33

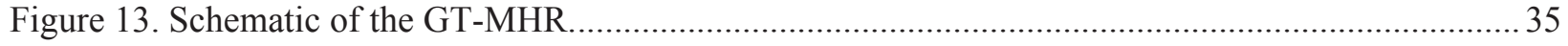

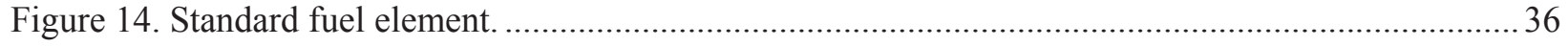

Figure 15. Bypass model relationship to GT-MHR ............................................................................. 39

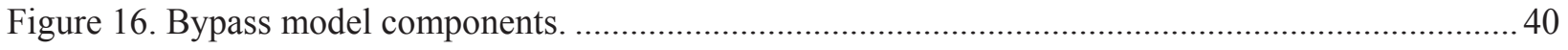

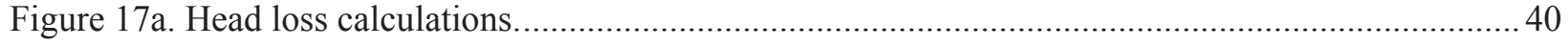

Figure 17b. Design head loss and Reynolds number estimates. ........................................................ 41

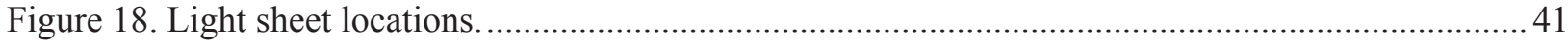

Figure 19. Actual gap spacing about the model cross-section. ............................................................ 42

Figure 20. Variation in (a) mean velocity and (b) mean turbulence stress versus sample size..................43

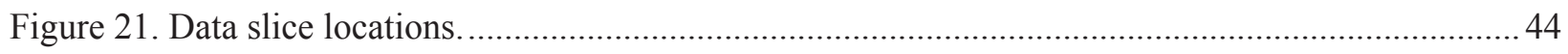


Figure 22. Typical velocity contour plot $(\mathrm{Q}=351.15 \mathrm{~L} / \mathrm{min}$ and $\mathrm{x}=-1029.5 \mathrm{~mm})$.

Figure 23. Typical flow area discretization of a coolant channel (Represented vectors are for illustrative purposes and do not accurately represent the flow along the axis of the tube).

Figure 24. Typical variation of time-averaged velocity in a coolant channel located at $[\mathrm{x}, \mathrm{z}]=[-1029.5,103]$. .46

Figure 25. Variation of time-averaged velocity in a gap located at $[x, z]=[-1029.5,0]$. .46

Figure 26. Superimposed velocity contour plot (superimposed data on upper 3 tubes). .47

Figure 27. Comparison of CFD and experimental data. .48

Figure 28. Isometric view of adverse pressure gradient flat plate study with turbulence generator. 49

Figure 29. Detail of the local seeding tube fastened near the leading edge of the flat plate. .50

Figure 30. Experimental data achieved in the center window of the MIR during the ZPG without a TG installed.

Figure 31. APG case having large FOV and mezzo FOV (by varying the dt between camera images).

Figure 32. ZPG with TG installed preliminary results: (a) the freestream turbulence intensity versus plate location, (b) Reynolds number based on x versus plate location, (c) freestream streamwise velocity component versus plate location, (d) boundary layer thickness versus plate location.

Figure 33. Comparison of direct numerical simulation with experimental results from the MIR flat plate experiment. 53

Figure 34. Screen shot from PIV-Online wiki.

\section{TABLES}

Table 1. Non-dimensional experimental parameters that affect performance. ..........................................20

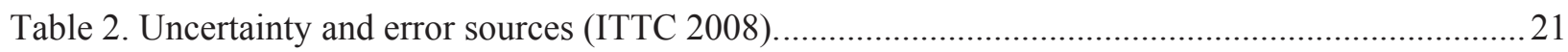

Table 3. Selected GT-MHR design parameters (General Atomics 1996)................................................. 36

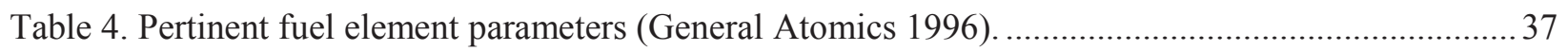

Table 5. Summary of CFD calculations for one-sixth sector of the bypass model (Johnson 2011)........... 38

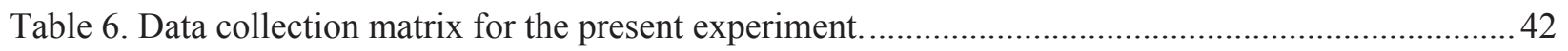

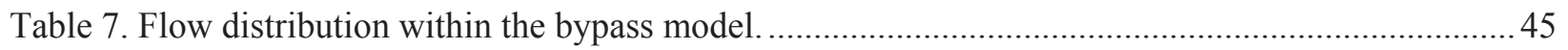

Table 8. Bypass flow results for a scaled 3-mm gap width (superimposed data on upper 3 tubes)...........47 


\section{ACRONYMS}

APG adverse pressure gradient

Cf coefficient

CFD computational fluid dynamics

DNS Direct Numerical Simulation

DPIV Digital Particle Image Velocimetry

FOV field of view

GT-MHR Gas Turbine-Modular Helium Reactor

INL Idaho National Laboratory

ITTC International Towing Tank Conference

IW interrogation window

MIR Matched Index of Refraction

PIV Particle Image Velocimetry

RPC Robust Phase Correlation

SA sensitivity analysis

SCC standard cross-correlation

SPIV stereo PIV

TFM turbine flow meter

TG turbulence generator

USU Utah State University

VT Virginia Technical University

ZPG zero pressure gradient 


\section{PIV Uncertainty Methodologies for CFD Code Validation at the MIR Facility}

\section{INTRODUCTION}

Significant challenges face thermal hydraulic design and modeling for advanced reactor concepts. There is a need for whole core system codes, which model the relevant physics and thermo-mechanics with local resolution to investigate the flow performance during operation and accident scenarios. Due to the complexity of the reactor core, only a portion of the core has been studied, specifically using computational fluid dynamics (CFD) to address key flow phenomenon of the lower plenum, core bypass, and the effects of graphite surface roughness (Johnson, Sato, Schultz 2010). CFD works well to investigate many types of flow behavior; however, for turbulent flow, it relies upon a single turbulence model to close the Reynolds Averaged Navier-Stokes equations and approximate a flow solution. In general, turbulence models have been developed to focus on a specific flow behavior, such as swirl, free shear flows, near wall flows, etc. Unfortunately, during reactor operation, many types of flow are present throughout the core, possibly invalidating the use of any one model. For this reason, it is critical to experimentally validate the turbulence models used in upcoming reactor designs with scaled thermal hydraulic data and known uncertainties.

The Matched Index of Refraction (MIR) flow facility at Idaho National Laboratory (INL) is used to produce experimental velocity data for CFD model validation. The data is obtained by performing separate effects experiments, which replicate a flow as it travels in and around representative core reactor geometry. Previous studies, including the lower plenum (McIlroy Year 2010) and bypass flow (Conder Year 2012) experiments, have produced validation data that require uncertainty quantification. These studies use a velocity measurement technique called Particle Image Velocimetry (PIV), and uncertainty quantification is required for stringent experimental data and CFD model validation. Unfortunately, the uncertainty in PIV measurements is difficult to determine, and a standard uncertainty quantification does not exist. As such, the accuracy of past MIR measurements is unknown. Thus, a standardized methodology is sought to quantify PIV uncertainty.

The main objective of this study is to discuss different uncertainty techniques being developed at Utah State (USU) and Virginia Technical University (VT) to quantify PIV uncertainty for the experiments being conducted in the MIR laboratory. The methodology described here permits future uncertainty quantification for MIR PIV experimentation for the purpose of data quality assessment. To do this, first a brief description of the MIR facility is given, including an overview of some important uncertainty factors. Second, the uncertainty associated with the MIR index matching technique is quantified. Third, a past experiment performed in the MIR facility is described, which requires PIV uncertainty quantification. Last, two PIV uncertainty methodologies are presented to evaluate the uncertainty of PIV in the MIR, and the details of the uncertainty methodology is described in detail in Appendix B.

\section{DESCRIPTION OF MIR FACILITY}

The MIR facility was constructed at INL to perform basic fluid dynamic research and measurement experiments, primarily using optical techniques such as PIV and Laser Doppler Velocimetry. It is the largest index-matching facility in the world and has been used to conduct a wide range of experiments, incorporating both flow visualization and measurement techniques, generally performed within complex models. The MIR facility also provides higher Reynolds numbers and increased spatial resolution compared to similar facilities. Stoots et al. (2001) provided a comprehensive review of the system, including an assessment of it capabilities; therefore, only a brief overview is provided here.

Submersing fused quartz models in light mineral oil permits the flow to be studied in and around complicated geometries. Figure 1 demonstrates how refractive index matching causes the quartz to disappear in mineral oil. 


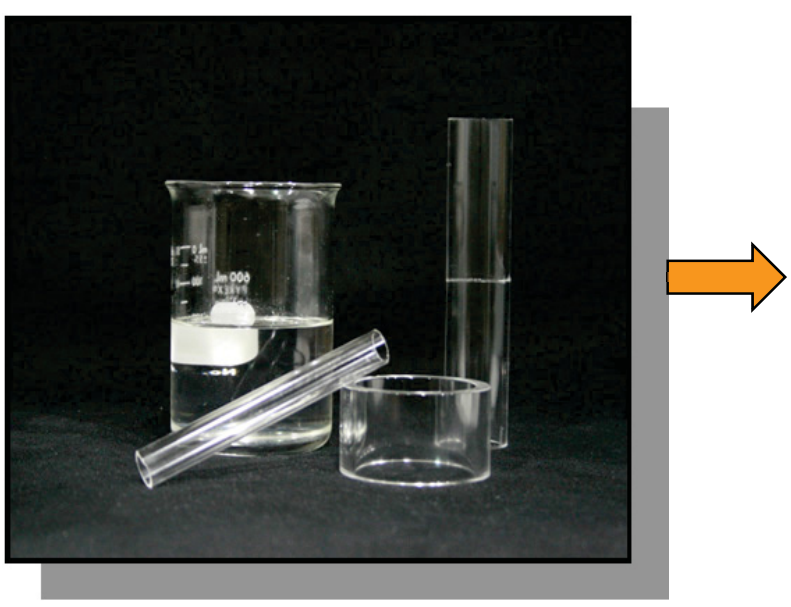

(a)

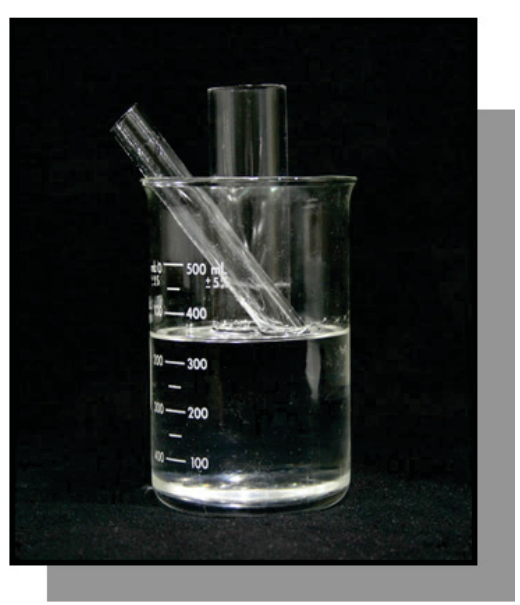

(b)

Figure 1. Index matching of quartz and mineral oil — part (a) demonstrates the quartz outside the oil, and (b) demonstrates the quartz while submersed in the oil.

The facility was modeled after a typical wind tunnel and was fabricated with a stainless steel frame. It consists of several components, including a settling chamber, a square contraction, and a test section, as shown in Figure 2. The settling chamber is comprised of a single stainless-steel honeycomb structure and several screens that straighten the flow and remove non-uniformities. The 4:1 square contraction is attached downstream of the settling chamber and produces nearly uniform flow at the entrance of the test section. Flow is driven into the test section by an axial pump that is powered by a $56 \mathrm{~kW}$ variable speed motor. At full capacity, this pump can drive nearly $0.63 \mathrm{~m}^{3} / \mathrm{sec}$ of mineral oil, achieving a maximum velocity of about $1.7 \mathrm{~m} / \mathrm{s}$ in the test section with a turbulence intensity of less than $0.5 \%$. The test section is $0.6-\mathrm{m}$ square and $2.43-\mathrm{m}$ long. It is made from a polycarbonate material, and contains large, optically clear glass windows to permit optical measurements of the flow.

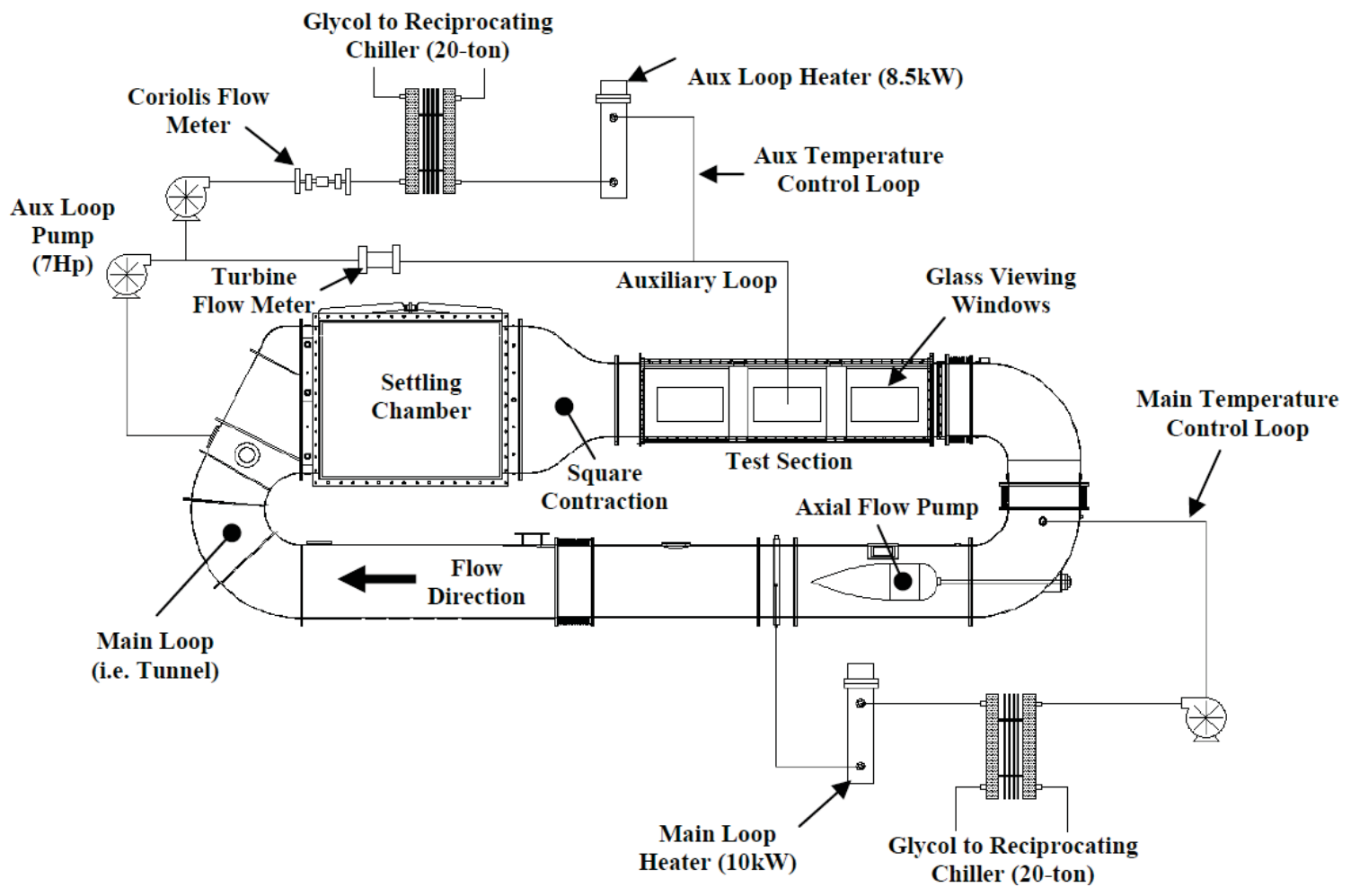

Figure 2. Matched Index of Refraction flow facility. 
The MIR was designed as an isothermal facility capable of sustaining a precise temperature in its test section. Mineral oil is extracted from the main tunnel into a temperature control loop at a flow rate of $269 \mathrm{~L} / \mathrm{min}$, where the oil is chilled by a glycol-cooled heat exchanger, reheated by a 10-kW resistance heater, and then plumbed back into the main system. When properly operated, the temperature within the test section can be controlled within $\pm 0.01^{\circ} \mathrm{C}$ of the index-matching temperature.

When flow is desired within a model, oil is extracted into an auxiliary loop by a 7-horsepower pump. This pump is capable of pushing 1500 LPM (with approximately 20 meters of pressure head) into a model, where the exact flow rate is measured using a calibrated turbine flow meter. For temperature control, a small portion of this oil is plumbed through an auxiliary temperature control system and is configured similarly to the temperature control loop. The flow through of this sub-loop is measured by using a self-calibrating Coriolis flow meter.

A traversing system is fitted along the test section, which permits translation of LaVision Imager Pro Plus PIV cameras to move in the vertical, stream-wise, and cross-stream directions. The traverse is operated by several programmable servo motors and is controlled through LabVIEW. The exact position of the PIV cameras is determined using calibrated linear encoders, accurate to $5 \mu \mathrm{m}$. It is important to note that the quartz models installed in the test section are aligned to the coordinate axis of the traversing system, generally to $0.01 \mathrm{in}$.

A Big Sky, twin, double-pulsed $532 \mathrm{~nm}$ neodymium-doped yttrium aluminum garnet laser is mounted below the test section where the light sheet is oriented in the streamwise direction and projected up through the bottom. Depending on the setup, one or two cameras focus on the model through lenses, typically between 20 and $105 \mathrm{~mm}$, at acute angles (less than 15 degrees) to the test section windows. Small angles are desired to minimize refraction at the test section air-to-window interface. Scheimpflug adapters are installed to give a sharp focus over the entire field of view (FOV).

Being outside the test section, the cameras look through air, glass, mineral oil, and quartz in order to view the flow within the model. With the exception of quartz and mineral oil, these media do not have similar refractive indices. To take measurements across the width of the model, optical refraction is addressed by coordinating the movement of the cameras relative to that of the laser. A method similar to McIlroy et al. (2011) is used to compensate for theses refractive issues. The camera-to-laser movement ratio is approximately 0.65 but depends on the camera angles achieved during PIV calibration.

The cameras are calibrated with precisely machined plates that consist of dots that fill the entire FOV; typically, a LaVision Type 7 or Type 20 calibration plate is used. As the cameras look through multiple media to see into the test section, a polynomial model is applied to map the FOV; after, self-calibration is performed for stereo measurements to correct any misalignment of the laser light sheet. The light sheet is typically adjusted to a thickness of $1 \mathrm{~mm}$. Measurements are realized using DaVis v.8.08 software.

During processing, an interrogation window (IW) is chosen based on the smallest feature size in the model to achieve at least 5-10 vectors resolution within. The flow is seeded such that at least 8-10 particle pairs are in each IW. The cameras collect data at a frame rate between 2 and $15 \mathrm{~Hz}$. The time delay between sequential frames is chosen based on the criteria set by Wilson et al. (particle displacements were limited to traveling between 25 and $62.5 \%$ of the IW) (Wilson 2009). When time averaging the measurements, the typical number of images is determined using a statistical method proposed by Uzol et al. 2001).

State-of-the-art measurement techniques, such as PIV, are being used at the MIR facility in an effort to better understand fundamental flow phenomena in nuclear reactor cores and their components. PIV is a non-intrusive velocity measurement technique that tracks flow by imaging the movement of small tracer particles within a fluid. The MIR uses a double-pulsed laser to illuminate the particles by projecting a thin laser light sheet into the flow domain, acting much like a camera flash. The particles are observed and imaged using a charged coupled device camera as they move with the flow field of the fluid. The images are interrogated to determine particle displacements, which are then used to calculate the velocity field. Figure 3 illustrates a general PIV setup. 


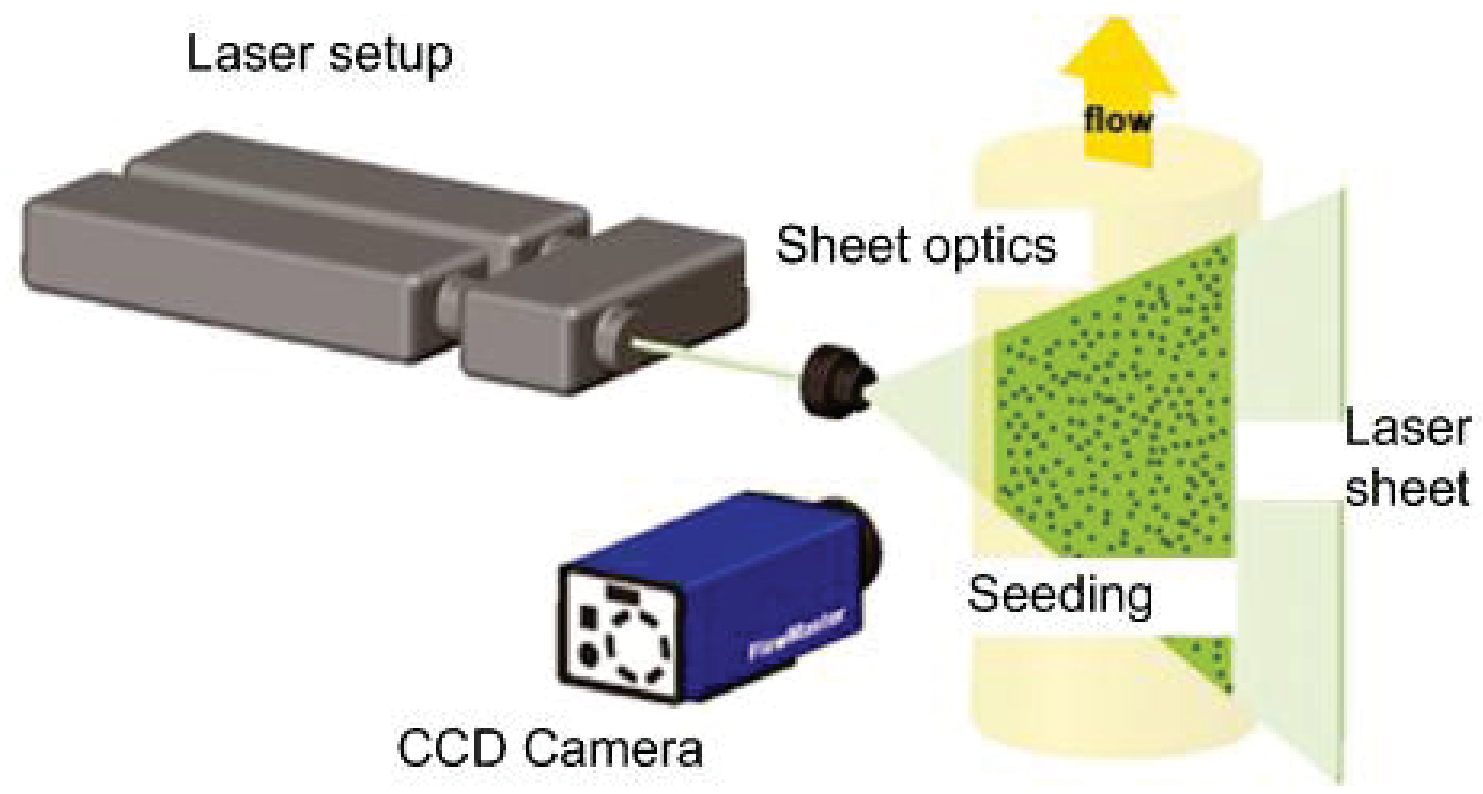

Figure 3. Particle Image Velocimetry.

Multiple images are recorded at a single FOV to calculate instantaneous and time-averaged velocity and turbulence quantities. Each image is divided into multiple interrogation regions or IWs where statistical correlation algorithms are employed to calculate the average particle displacement (Adrian and Westerweel Year 2010). The corresponding displacement map is then used to calculate a velocity field. It is important to understand that the particles are not tracked; the cross-correlation simply returns the most likely displacement, represented by the highest peak of the field.

At the heart of the PIV calculation is the cross-correlation algorithm, which is used to estimate the displacement of particles in each IW. The algorithm generates a correlation plane for each window that defines the magnitude and direction of the particle movement. A typical plane is illustrated in Figure 4. The particle displacement is indicated by the location of the largest peak relative to the center of the IW (Adrian and Westerweel Year 2010).

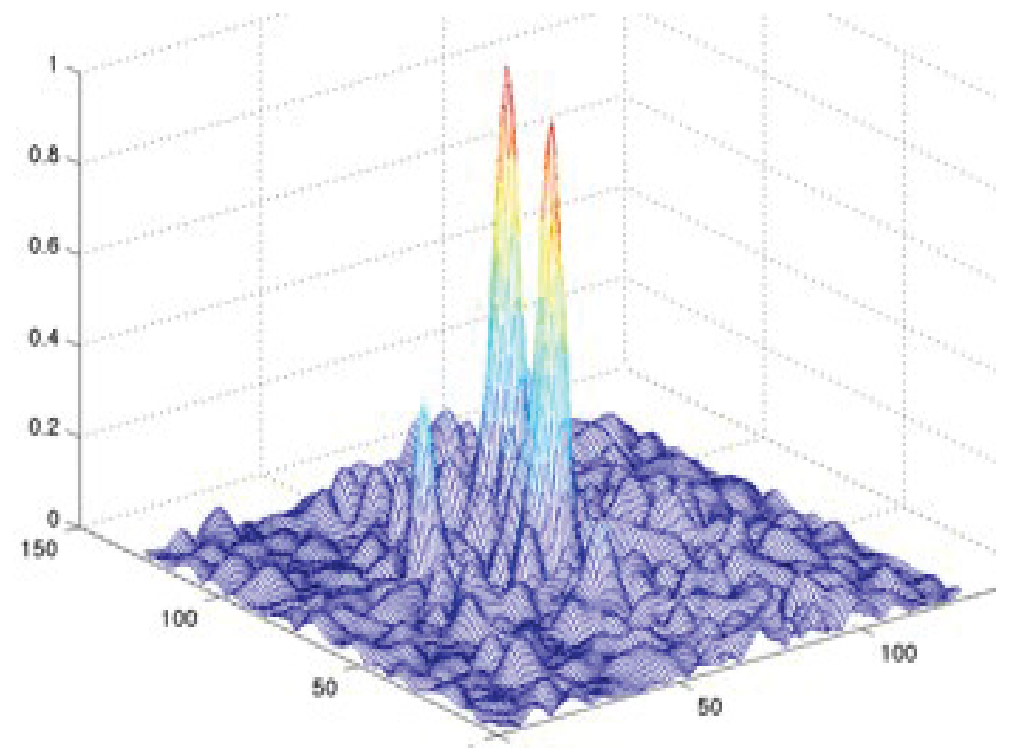

Figure 4. Representative view of PIV correlation plane. 
Over the past 20 years (Adrian 2005), many efforts have been directed towards the refinement of the basic digital algorithms developed by Willert and Gharib (1991). Some key developments that significantly impacted the applicability of PIV include discrete window offset (Westerweel and Gharib 1997; Wereley and Meinhart 2001), window deformation (Astarita 2007; Nobach and Tropea 2005; Scarano 2002), and iterative multigrid and super-resolution methods (Scarano and Riethmuller 1999; Scarano and Riethmuller 2000; Scarano 2003; Scarono 2004). Three-component planar velocity measurements are accomplished using a standard stereo-Digital Particle Image Velocimetry (DPIV) methodology (Wieneke 2005).

A wide variety of specialized approaches have been developed to address particular limitations of the method. For example, techniques such as the ensemble correlation (Meinhart, Wereley, and Santiago 2000) and the single pixel correlation (Westerweel, Geelhoed, and Lindken 2004), were developed for micro-DPIV applications and have been shown to offer greater accuracy when evaluating certain steady or periodic flows. Although a vast amount of research is dedicated to the development of advanced algorithms, improvements on the fundamental processing technique are still needed (Adrian 2005). In particular, for cases with strenuous experimental conditions where illumination may be limited or nonuniform, or in the vicinity of boundaries and under high-shear rates, the accuracy of the standard cross-correlation (SCC) is severely compromised, thus limiting its capability for evaluating more complex flow fields (Scarano 2002). In addition, the accuracy of particle position subpixel interpolation schemes has been the subject of several investigations (Marxen et al. 2000; Udrea et al. 1996). Gaussian fitting schemes, such as the three-point estimator and local least-squares estimator, have been seen to provide reduced error in position estimation of the geometric image compared to more traditional methods such as center of mass (Willert and Gharib 1991; Marxen et al. 2000; and Udrea et al. 1996). In addition, peak-locking was significantly reduced for the three-point fit when compared to a centroid approximation (Willert and Gharib 1991). The use of Gaussian fitting schemes for finding particle positions and correlation peaks has been very well documented. However, using the same schemes to find particle or correlation diameter has not been investigated comprehensively. Marxen et al. (2000) classified the measured particle diameter with the true diameter for a three-point Gaussian estimator and a least-squares estimator. Their results were significantly affected by preprocessing methods, and errors in excess of 50\% were reported. However, since this issue was not the primary focus of their study, sizing of the particle images did not receive further attention. Recent work proposed improved Gaussian fitting schemes that deliver better accuracy in both positions and diameter estimation (Brady, Raben, and Vlachos 2009).

The velocity measurement dynamic range for PIV has been one of the main challenges of the method (Adrian 2005). Standard PIV can achieve a dynamic range of about 200:1 between the highest resolvable velocities and the slowest (where the noise swamps the measurement) (Adrian 1997). To overcome this difficulty, several researchers have investigated combining cross-correlations between more than one pair of images into a single velocity field, thereby enhancing the accuracy of the lower velocity regions of each field (Hain and Kähler 2007; Pereira et al. 2004; Persoons O’Donovan, and Murray 2008; and Stanislas et al. 2008).

Several studies have explored the potential of advanced windowing techniques. Amongst them, the most common is the use of zero padding, which removes the periodic effect of the Fourier-based cross-correlation (Raffel Willert and Kompenhans 1998). However, the use of zero padding has been met with mixed results, primarily because of errors introduced by the window discontinuity. Fore and Tung (2005) demonstrated that the effect of this discontinuity can be substantially attenuated through proper background scaling and noise removal. Alternative windowing techniques, such as the Gaussian window mask, are able to attenuate some of these Fourier-based effects without introducing a discontinuity. Gui and Merzkerch (2000) applied a Gaussian window mask to the image regions in order to reduce bias errors introduced by the loss of correlation. Nogueira, Lecuona, and Rodriguez (1999) demonstrated how the Gaussian mask can be used to alter the low pass filtering effect of the DPIV estimation. Scarano (2003) extended this idea through the use of elliptical Gaussian masks aligned to minimize the curvature of the flow. 
Accurate PIV measurements are achieved when careful consideration is given to the methods and parameters used to capture and process the PIV images. Important factors include the frame delay (time delay between sequential images), sample size (number of vectors), particle density/seeding (number of seeding particles within each interrogation region), and image processing methods.

A literature review was conducted to determine the major parameters that contribute to PIV uncertainty. This is by no means a comprehensive list, but it represents a compilation of some of the variants that have been studied by past researchers. Adrian and Westerweel (2010) present an outstanding review of particle-imaging techniques and define many important parameters used during data acquisition. These parameters include magnification, particle image diameter, depth of field, and source density. Keane and Adrian (Year 1992) studied performance of auto-correlation methods and cross-correlation methods of interrogation of single-exposure and double-exposure double-pulse PIV systems. They identified a number of non-dimensional experimental parameters that affect these measures of performance. The authors used an extended range of velocity fields in their analysis and a Monte Carlo simulation of the cross-correlation method to reveal the critical dimensionless parameters of the mean cross-correlation function and its fluctuations that effect performance. These parameters are shown in Table 1.

Table 1. Non-dimensional experimental parameters that affect performance.

\begin{tabular}{|l|l|}
\hline \multicolumn{1}{|c|}{ Symbol } & \\
\hline $\mathrm{x}$ & Location in the fluid \\
\hline $\mathrm{u}\{\mathrm{x}\}$ & Local stream wise velocity \\
\hline $\mathrm{W}$ & Local span wise velocity \\
\hline $\mathrm{M}$ & Lens magnification \\
\hline $\mathrm{C}$ & Mean concentration of seeding particles \\
\hline $\mathrm{d}_{\mathrm{I}}$ & Size of IWs \\
\hline $\mathrm{d}_{\mathrm{t}}$ & Particle image diameter \\
\hline $\mathrm{N}_{\mathrm{I}}$ & Particle image density or mean number of particles in the interrogation spot \\
\hline $\mathrm{Dz}$ & Laser sheet thickness \\
\hline $\mathrm{Dt}$ & Time interval between successive images. \\
\hline $\mathrm{N}_{\mathrm{I}}$ & Particle image density \\
\hline $\mathrm{D}_{0}$ & Signal-to-noise ratio in the resulting correlation function \\
\hline
\end{tabular}

The International Towing Tank Conference (2008) published recommended procedures and guidelines for uncertainty analysis of PIV. These procedures and guidelines describe a reasonable approach to estimating uncertainty in PIV measurements by separating the analysis into four categories: calibration, flow visualization (seeding, laser pulsing), image detection (laser synchronization, image quality, contrast ratio), and data processing. The conference identified error sources from calibration procedures and techniques, image distortion, positional accuracy, laser power fluctuations, correlation peak identification, mis-matching, sub-pixel accuracy, pulse timing accuracy, position accuracy, and magnification. This conference found that the largest uncertainty was in comparison of calculated vectors with surrounding vectors (mis-match). The next two largest sources of uncertainty were sub-pixel analysis and calibration uncertainty. A brief summary of the described uncertainty and error sources are shown in Table 2. 
Table 2. Uncertainty and error sources (International Towing Tank Conference 2008).

\begin{tabular}{|l|l|}
\hline \multicolumn{1}{|c|}{ Category } & \multicolumn{1}{c|}{ Uncertainty and Error Sources } \\
\hline \multirow{4}{*}{ Calibration } & Image distance of calibration plate dots \\
\cline { 2 - 2 } & Physical distance of calibration plate dots \\
\cline { 2 - 2 } & Image distortion caused by lens \\
\cline { 2 - 2 } & $\begin{array}{l}\text { Misalignment and/or distance between } \\
\text { laser sheet and calibration plate }\end{array}$ \\
\cline { 2 - 2 } & Lens aberration and magnification \\
\cline { 2 - 2 } Particle Image & Camera chip distortion \\
\cline { 2 - 2 } & Laser power fluctuation \\
\cline { 2 - 2 } & Camera viewing angle \\
\cline { 2 - 2 } & Sub-matching of paired particle images \\
\hline Time Interval & Camera and Laser pulse timing \\
\hline Experiment & Particle lag \\
\cline { 2 - 2 } & Out-of-plane velocity component \\
\hline Measurement & Origin correlation \\
\cline { 2 - 2 } Position & Particle distribution (uniformity) \\
\hline
\end{tabular}

At a recent PIV Uncertainty Workshop (2007) organized by INL, the participants agreed that a basic uncertainty analysis should always include an assessment of particle lag, calibration error, grid convergence, velocity gradient random error, and convergence of mean and fluctuating quantities. They noted that uncertainty sources can be categorized according to the particles, their images, or the velocimetry computations.

Guezennec and Kiritsis (1990) investigated the influence of image contrast, image noise, particle density, particle-size distribution, and particle displacement on the accuracy of PIV velocity measurements. The authors studied images that were generated according to a specific set of parameters that included the number of particles in an image, the mean intensity level of the particle and background pixels, the mean radius of the particles, and the standard deviation of the particle radius and the image noise. The authors claimed that choosing the "best" threshold value for every image is probably the most important task in obtaining velocity measurements with PIV. Huang, Dabri, and Gharib (1997) conducted a study to quantify and reduce errors in 2-D PIV. The authors suggest that rms-error is a function of the size of the IW, number of particles, local velocity gradient inside the IW, non-matching particles, and noise.

Uzol and Camci (2001) examined the effect of sample size, turbulence intensity, and the velocity field on the experimental accuracy of ensemble-averaged PIV measurements. The authors found that increasing sample size resulted in significant reduction in the data scatter. Ullum et al. (1998) assessed PIV measurement accuracy and established the need for large sample sizes, particularly for higher order statistics.

Additionally, McEligot et al. (2007) quantified the measurement uncertainty of PIV for velocity, positioning, fluid property, geometry, flow rate, and Reynolds number parameters for the lower plenum experiment.

The tracer particles in an ideal experiment need to have the same motion as the fluid. For a single particle, smaller than the fluid scale of motion, the force balance from Newton's second law could be written as:

$$
m_{p} \frac{d v_{p}}{d t}=\text { Net buoyant force }+ \text { Quasi steady viscous drag + Viscous force + Added mass + Fluid stress } .
$$



2007):

Seeding particles used for PIV technique have the following important properties (PIV Workshop

- They do not affect flow properties

- They are randomly distributed across all elements of fluid

- They follow the flow without changing properties

- The larger the particle, the more light it scatters (better images)

- Smaller particles directly correlate to improved tracking of the particles

- They do not interact with each other.

The main objective of the PIV is to determine fluid velocity, which is inferred from the motion of tracer particles. The velocity is measured indirectly as a displacement $D\left(X ; t^{\prime}, t^{\prime \prime}\right)$ of the tracer particles in the finite time interval $\Delta t=t^{\prime \prime}-t^{\prime}$ explained by Westerweel (1997) as:

$D\left(X ; t^{\prime}, t^{\prime \prime}\right)=\int_{t^{\prime}}^{t^{\prime \prime}} v[X(t), t] d t$.

For ideal tracer particles, the tracer velocity $v$ is equal to the local fluid velocity $u(X, t)$. The displacement field obtained from Equation (1) only provides information about the average velocity along the trajectory over a time $\Delta t$ (i.e., D cannot lead to an exact value of $u$ ) but approximates it within a finite error $\epsilon$ (Westerweel 1997):

$\|D-u . \Delta t\|<\epsilon$

The error is negligible if the spatial and temporal scales of the flow are large with respect to spatial resolution and exposure time delay. The displacement field should always be sampled at a density that matches the smallest length scale of the spatial variations in D.

The seeding/tracer particles constitute a random pattern that is tied to the fluid and the fluid motion, which is visible through changes of the tracer pattern. The tracer pattern in $\mathrm{X}$ at time $\mathrm{t}$ could be defined as: (Westerweel 1997):

$G(X, t)=\sum_{i=1}^{N} \delta\left[X-X_{i}(t)\right]$

where $\mathrm{N}$ is the total number of particles in the flow, $\delta(X)$ is the Dirac delta function, and the $X_{i}(t)$ is the position vector of the particle with index $i$ at time t. Integrating $G(X, t)$ over a volume yields the number of particles in that volume.

Adrian (1991) presents an outstanding review of particle-imaging techniques and defines many of the key parameters used in PIV analysis. These parameters include:

- Magnification: $M(z)=\frac{d_{i}}{d_{0}-z}$, where $d_{i}$ is the distance from the lens to the image plane, $d_{\mathrm{o}}$ is the distance from the lens to the object plane, and $z$ is the distance away from the object plane.

- Point response function: $d_{s}=2.44(1+M) f^{\#} \lambda$ where $M$ is the magnification, $f^{\#}$ is the $f$-number of the lens, and $\lambda$ is the laser wavelength.

- The particle image diameter: $d_{\tau}=\sqrt{{M_{o}}^{2} d_{p}{ }^{2}+d_{s}{ }^{2}+d_{a}{ }^{2}}$, where $d P$ is the particle diameter.

- Source Density: $N_{s}=C . \Delta z_{0} \frac{\pi \cdot d_{\tau}{ }^{2}}{4 . M^{2}}$, where C is the number density of the seeding, $\Delta z_{0}$ is the intensity of the light sheet thickness, $M^{2}$ is the image of magnification, and $d_{\tau}$ is the particle image diameter. 
Choosing the best threshold value for every image is probably the most important task in obtaining velocity measurements with PIV, as explained by Guezennec and Kiritsis Guezennec and Kiritsis (1990). Thresholding is the process of separating particles from the background noise - getting rid of the outliers. Guezennec and Kiritsis (1990) also found that, for images with good contrast $(C \geq 100)$, the effect on velocity measurement error is insensitive to the noise level of the images. Additionally, the authors show that the average particle position error and the standard deviation of the error increase as the number of particles or the average size of the particles increase. However, they state that by obtaining a sharply focused light sheet, an accuracy of 0.1 to 0.2 pixels is possible.

Westerweel (1997) showed that the measurement resolution is not determined by the pixel size, but by the particle-image diameter relative to the size of the interrogation region, and further improvements could be obtained by optimizing the subpixel interpolation with respect to the shape of the discrete displacement correlation peak.

\subsubsection{Uncertainties in Velocity Measurement}

As explained previously, the basic measurement of a PIV system is an instantaneous velocity component deduced from the translation $\Delta \mathrm{x}_{\mathrm{p}}$ of a group of particles over a time interval $\Delta \mathrm{t}$ :

$\mathrm{V}_{\mathrm{P}}=\mathrm{S} \frac{\Delta \mathrm{x}_{\mathrm{p}}}{\Delta \mathrm{t}}$

where $\mathrm{S}$ is the scaling factor, $\Delta \mathrm{x}_{\mathrm{p}}$ is the displacement in pixels, and $\Delta \mathrm{t}$ is the time between images. The basic percent uncertainty in the velocity can be represented as:

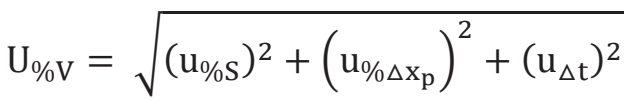

The propagation of such uncertainties into the mean statistics reported depends on the extent to which the uncertainties are random (precision) or systematic (bias) and the manner in which the results are presented. In some situations, fixed uncertainties can be removed from the presentation by normalizing the quantities involved. The averaging process for determining mean statistics will reduce the resulting percent uncertainties when they are random.

Huang et al. (1997) conducted a study to quantify and reduce, when possible, errors in 2-D PIV. The authors claim that with 20 particles in an interrogation area of $32 \times 32$ pixels, bias and rms errors vary from about 0.025 to 0.1 pixels for displacements $(\Delta x)$ of 1 to 10 pixels. With an interrogation area of $16 \times 16$ pixels and 200 particles, these uncertainties fall in a range of about 0.1 to 0.2 pixels up to a displacement $(\Delta x)$ of 7 pixels, and then they explode. By employing an adaptive multipass technique, first with an interrogation area of $64 \times 64$ pixels and then one of $32 \times 32$ pixels, bias and rms errors are reduced to about 0.03 pixels for up to 10 pixel displacements. The authors suggest that rms-error is a function of the following factors:

- $\quad$ Size of IW

- Number of particles (best $>10$ ) per interrogation area

- Local velocity gradient inside IW

- Nonmatching particles $(\mathrm{Vz})$ and all sorts of noise.

LaVision (2002) also suggests that rms-error (random uncertainty) is typically 0.05 to 0.1 pixels in real data. LaVision notes that a particle image diameter of less than 1 pixel can cause bias during vector calculation. They recommend particle sizes of at least 2 pixels. Slight defocusing and/or better algorithms, such as window deformation, can help with particle size. 
The processing algorithm for the LaVision PIV system gives uncertainties in displacements of about 0.05 pixels for synthetic images. Additional factors involved for images of real experiments include:

- Calibration

- Focus

- Displacement of particles

- Particle seeding

- Spatial gradients within each interrogation spot

- Image contrast

- Operator settings.

Therefore, the value of 0.02 pixels can probably be considered to be about the best possible pixel displacement under ideal circumstances.

To convert the basic PIV observation to actual velocities, a scaling factor (S) is employed, relating the pixel dimensions to physical distance in the FOV with units of pixel $/ \mathrm{mm}$. This quantity can suffer from both systematic (or fixed) and random uncertainties. The physical distance can be provided from a calibration device, fabrication sketches with tolerances, as-built drawings, and/or independent measurements. The connection to the pixel grid is obtained via the camera view for the same location(s). Conceptually, using a calibration procedure with a calibration device can account for parallax in the camera views. Since several of these measurements are accomplished optically, they can be subject to refractive index variations, which can be either systematic or random, depending on the temporal and spatial scales involved.

For stereo PIV (SPIV) with two cameras, a LaVision calibration plate of known distances, along with related software, is employed to relate the observed image dimensions in pixels to physical distances in the MIR oil tunnel. This determination can be used to calculate a scale factor in pixels $/ \mathrm{mm}$ or vice versa.

\subsubsection{Error Sources and Propagation of Errors}

Error sources and propagation of errors in PIV measurements at MIR facility are listed as follows (PLN-3669, "Matched-Index-of-Refraction MHTGR Prismatic Block Bypass Flow Control and Measurement Plan"):

- Temperature

- Oil properties

- Geometry

- Scaling factor-calibration and uncertainty

- Image distortion by lens

- Image distortion by charged coupled device camera

- Light sheet alignment to the calibration plate

- Particle image displacement (acquisition and reduction)

- Laser power fluctuation (spatial and temporal fluctuations)

- Viewing angle

- Particle motion. 
The main technical benefit of this effort is to significantly improve data quality of the MIR facility, and the sensitivity study will improve the ability of the MIR/PIV technique to collect high-quality data with less effort by concentrating on important parameters. It will make the whole experimental process in the MIR facility much more efficient and productive. Figure 5 summarizes the source of uncertainty in the MIR facility.

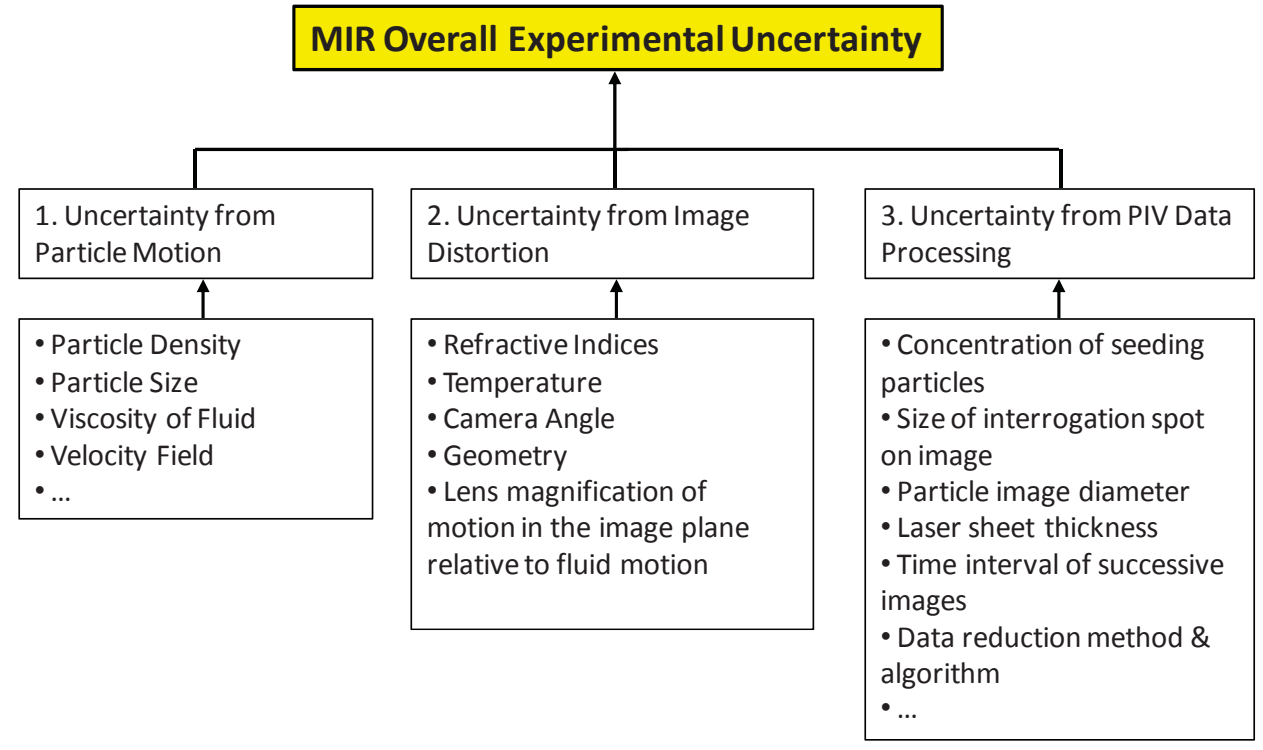

Figure 5. Identified uncertainty propagation structure and sources in MIR facility.

\subsubsection{Error versus Uncertainty}

There is a very significant difference between error and uncertainty. Error has a magnitude that is well defined, whereas uncertainty is an estimate (sometimes given in range [i.e., $\pm 5 \%]$ ). The error of a specific measurement source is a quantity with exact magnitude and specific signs expressing the difference of the measured from the true value. The uncertainty is the estimate of $\pm x$ of the range of the values around the measurement that contains the true, actual, but unknown, error(s) $\delta$.

\subsection{Summary}

PIV is a nonintrusive, state-of-the-art, velocimetry measurement technique with a very high spatial resolution. This technique is being used to benchmark CFD codes to improve the safety of current and advanced nuclear energy systems. PIV does not have an accepted uncertainty quantification methodology, which is required for rigorous code verification and validation. PIV will also establish INL's reputation and lead in verification and validation efforts.

\section{EFFECT OF OIL TEMPERATURE ON PIV MEASUREMENT ERROR IN THE MIR FACILITY}

The effect of oil temperature on PIV measurement error in the MIR facility was studied to estimate PIV measurement error in the MIR facility when the operating temperature deviated from the matching temperature. The mineral oil temperature in the MIR facility is very important because the refractive indices between the mineral oil and the quartz models are matched by adjusting temperatures. The difference of refractive indices generally becomes larger as the temperature is deviated from the matching temperature. The different indices of refraction distort optical images of cameras and finally cause measurement errors. 


\subsection{Uncertainty Propagation of MIR Facility}

Figure 6 shows the uncertainty propagation in the MIR facility. Since the velocity field in this facility is measured by the PIV technique, the uncertainty propagation is basically the same here as the general PIV methods. The PIV is an indirect measurement technique. To measure fluid velocities, the fluid is seeded by small particles, and the particle movements are detected instead of fluid movement itself. Since the particle movement might not follow the fluid movement exactly, depending on the particle/fluid properties and the flow conditions, it is obvious that there are uncertainties between the actual fluid velocity and the measured particle velocity. Generally, it is considered that the particle follows the fluid movements well in the absence of strong swirl flows or gravity force. Another important uncertainty is generated when the particle images are taken by optical cameras. This uncertainty is mainly caused by different refractive indices between fluids, windows, and models. Once the images are collected, the main uncertainties are caused by a data process that converts image data to particle velocities. This is a time-consuming job using various mathematical correlation methods. The uncertainties of this process have been previously investigated (McEligot et al. 2007).

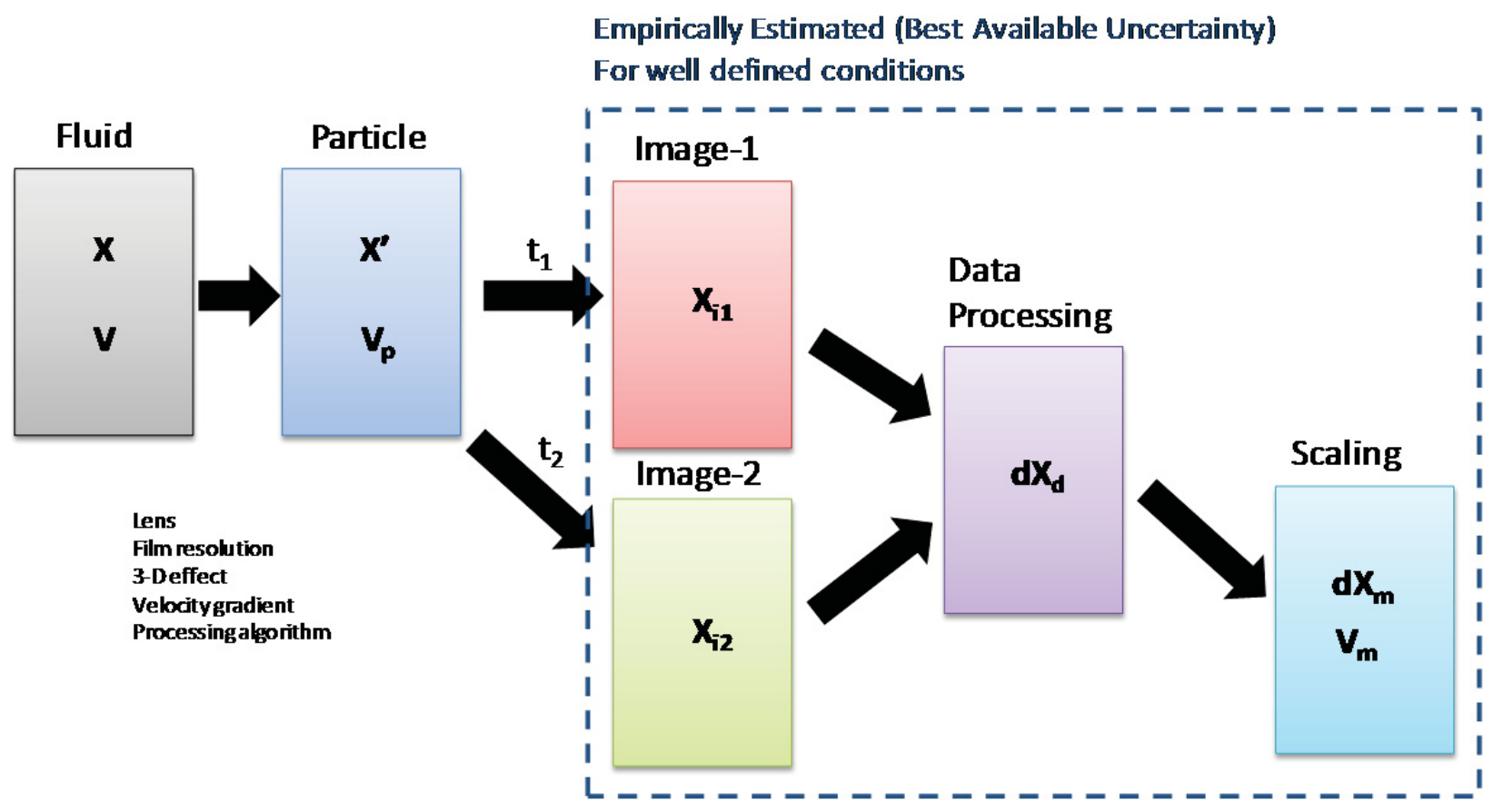

Figure 6. Uncertainty propagation of MIR facility.

This study focused on the optical uncertainty, which was not well investigated earlier in the MIR facility. The objectives of this study were to:

- Develop methods for quantifying optical uncertainty in the MIR facility

- Learn the effect of mineral oil temperature on the velocity measurement errors

- Learn the effect of various parameters on the velocity measurement errors

- Understand the importance estimation of various parameters on the velocity measurement. 


\subsection{Estimation Method of Velocity Error by Mineral Oil Temperature}

Figure 6 illustrates the basic technique to estimate optical errors (from Particle to Image-1 and Image-2, shown in Figure 5) in the MIR facility. The basic idea was to map the real object (X) to the image plane $\left(\mathrm{X}_{1}\right)$ for the reference conditions and settings. These conditions and settings represent the calibrating conditions. After that, change the temperature of the mineral oil in the facility and map the object (X) to the new image plane $\left(\mathrm{X}_{2}\right)$ to obtain mapping curves (see top center of Figure 7). By differentiating the two mapping curves, the following correlations were obtained:

$$
\begin{aligned}
& \frac{d x_{1}}{d x}=\frac{d x_{1} / d t}{d x / d t} \quad \text { (from X to X1) } \\
& \frac{d x_{2}}{d x}=\frac{d x_{2} / d t}{d x / d t} \text { (from X to X2) }
\end{aligned}
$$

By dividing $\mathrm{dx}_{1} / \mathrm{dx}$ by $\mathrm{dx}_{2} / \mathrm{dx}$, the following correlation was obtained:

$$
\frac{d x_{2}}{d x_{1}}=\frac{d x_{2} / d t}{d x_{1} / d t}=\frac{V_{2}}{V_{1}}
$$

This correlation represents the velocity ratios between actual measurement conditions and ideal (reference) conditions. It indicates how much actual velocity data are distorted from the calibrated data.

Figure 8 shows the basic equation set up for mapping an object plane $(\mathrm{X})$ to an image plane $\left(\mathrm{X}_{1}\right)$. The object plane represents the target that needs to be measured. The image plane represents the image mapped through the camera. This figure only shows the mapping in x-direction, but the same correlations can be applied to the other directions. Region 1 is air, Region 2 is a glass window, and Region 3 is mineral oil. In the real analyses, a quartz layer was inserted into Region 3 by separating the whole area into the following five regions:

- Region 1: from camera to window (air)

- Region 2: window (glass)

- Region 3: from window to model (oil)

- Region 4: model (quartz)

- Region 5: from model to measurement location (oil). 


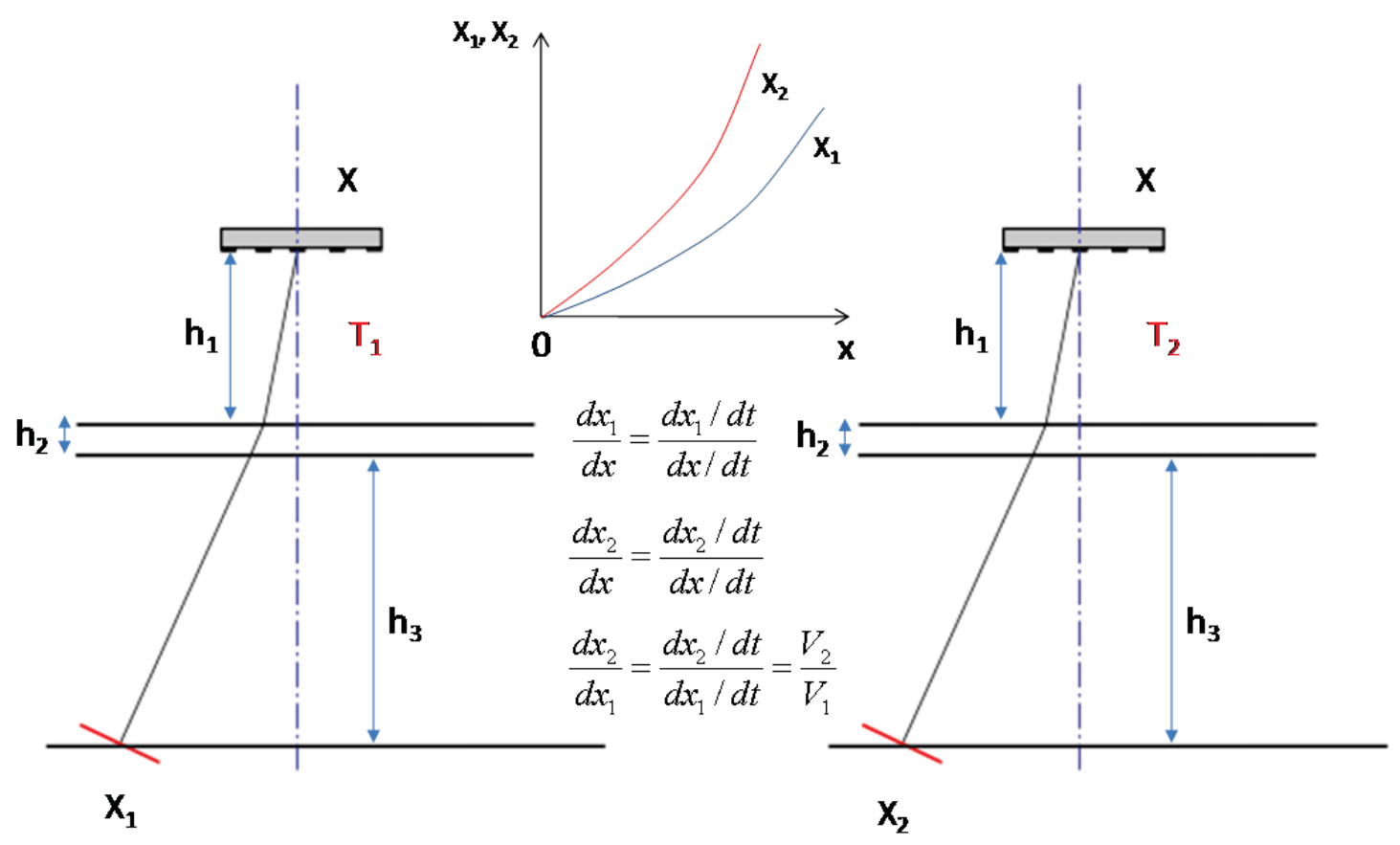

<Reference: Calibration>

$<$ Experiment $>$

Figure 7. Basic idea of estimating velocity error from deviated indices of refractions.

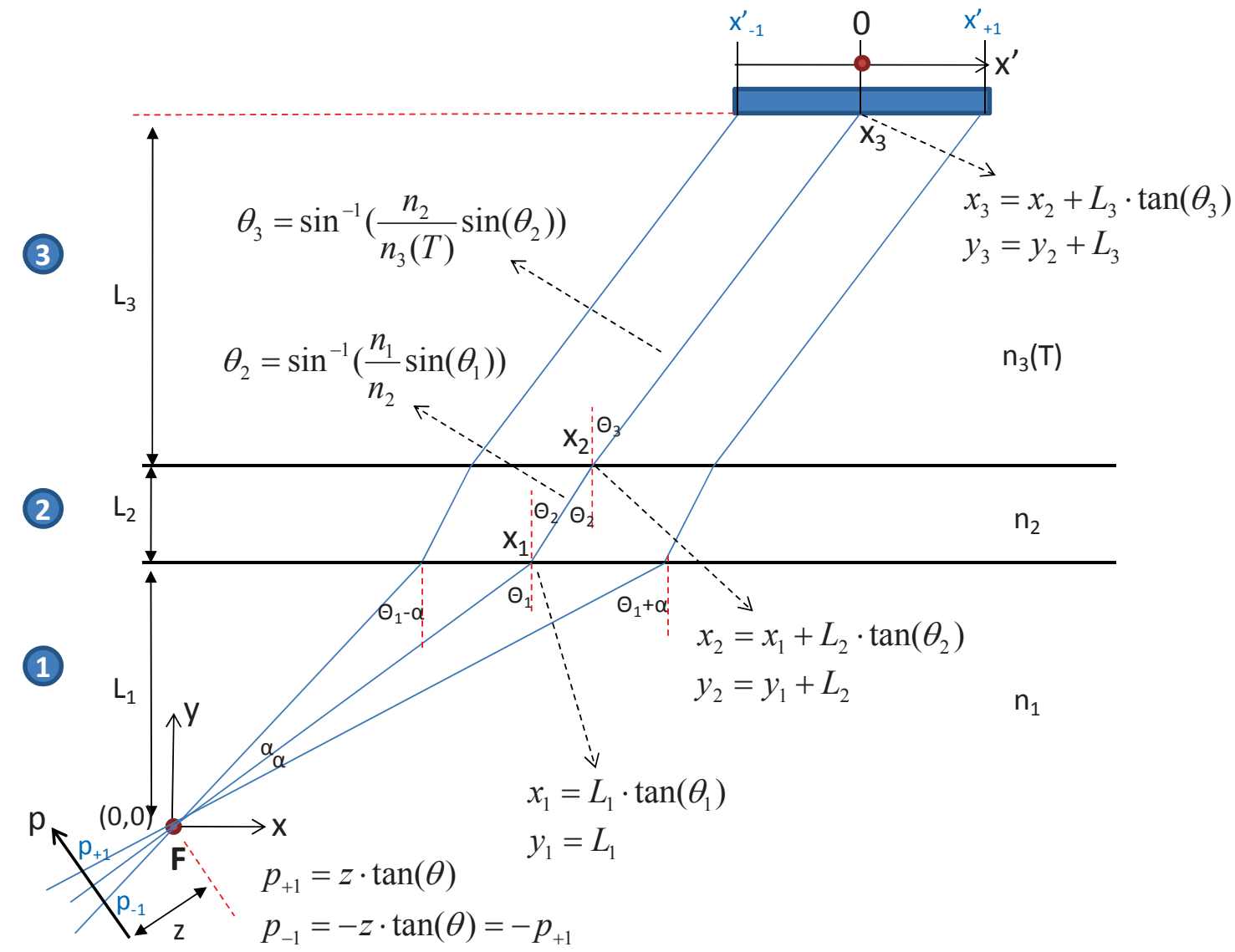

Figure 8. Equation setup for mapping object to image plane. 


\subsection{Analyses Method (Global Sensitivity Analyses)}

This section summarizes the uncertainty and sensitivity analysis (SA) of the PIV measurement error caused by oil temperature. This study used a random sampling Monte Carlo-based method called Global SA, which is briefly summarized below.

According to the definition by Satelli et al. (2004), SA is the study of how the variation in the output of a model contributes to different source of variations and of how the given model depends on the information fed into it. Originally, SA was created to deal simply with uncertainties in the input variables and model parameters. Over the course of time, the idea has been extended to incorporate model conceptual uncertainty (uncertainty in model structures, assumptions, and specifications). As a whole, SA is used to increase the confidence in the model and its predictions by providing an understanding of how the model response variables respond to changes in the inputs. Therefore, SA is closely related to uncertainty analysis, which aims to quantify the overall uncertainty associated with the response as a result of uncertainties in the model input.

SA can be categorized with the following different types of analyses:

- Screening

- Local sensitivity study

- Global sensitivity study.

The screening method, which has a huge number of input factors, is useful when the model is expensive to compute. The screening method is aimed at identifying a subset of input factors that is most likely to have a strong effect on the model output. Therefore, this method is approximate with low computational effort.

The local sensitivity study focuses on the local (point) impact of the factors on the model. This analysis is usually carried out by computing partial derivatives of the output functions with respect to the input factors. The local sensitivity study approach is practicable when the variation around the midpoint of the input factors is small and the trend is linear. When significant uncertainty exists in the input factors, the linear sensitivities alone are not likely to provide a reliable estimator of the output uncertainty in the model.

The global sensitivity study focuses on apportioning the input uncertainty to the uncertainty in the input factors. Typically, global SAs are based on the sampling-based method to quantify the influence of uncertain input parameters on the response variability of a numerical model. There are several different methods that can be used for a global SA: standard regression coefficient, Pearson product moment coefficient, Spearman coefficient, measure of importance (Sobol, Fast, etc.), and others. The global SA is generally based on the variance-based method. The following section gives the details.

\subsubsection{Variance-Based Methods}

This study used variance-based methods for SAs based on the variance decomposition. When the input variables are mutually orthogonal, independent, or noncorrelated, it is possible to decompose the variance of $\boldsymbol{f}(\boldsymbol{X})$ into terms of increasing dimensionality (Sobol 1990 and 1993) using:

$\mathrm{V}(\mathrm{Y})=\sum_{\mathrm{i}} \mathrm{V}_{\mathrm{i}}+\sum_{\mathrm{i}<j} \mathrm{~V}_{\mathrm{ij}}+\sum_{\mathrm{i}<j<k} \mathrm{~V}_{\mathrm{ijk}}+\mathrm{V}_{1,2, \ldots \mathrm{d}}$

The terms $V_{i}, V_{i j}, V_{i j k}, \ldots$, are called partial variance. These terms can be computed, for instance, as:

$\mathrm{V}_{\mathrm{i}}=\mathrm{V}\left[\mathrm{E}\left(\mathrm{Y} \mid \mathrm{X}_{\mathrm{i}}\right)\right]$

$\mathrm{V}_{\mathrm{ij}}=\mathrm{V}\left[\mathrm{E}\left(\mathrm{Y} \mid \mathrm{X}_{\mathrm{i}}, \mathrm{X}_{\mathrm{j}}\right)\right]-\mathrm{V}_{\mathrm{i}}-\mathrm{V}_{\mathrm{j}}$

$\mathrm{V}_{\mathrm{ijk}}=\mathrm{V}\left[\mathrm{E}\left(\mathrm{Y} \mid \mathrm{X}_{\mathrm{i}}, \mathrm{X}_{\mathrm{j}}, \mathrm{X}_{\mathrm{k}}\right)\right]-\mathrm{V}_{\mathrm{i}}-\mathrm{V}_{\mathrm{j}}-\mathrm{V}_{\mathrm{k}}$

The decomposition in Eq. (4) has the useful property that all the terms in Eq. (4) sum to 1 using:

$\sum_{\mathrm{i}} \mathrm{S}_{\mathrm{i}}+\sum_{\mathrm{i}<j} \mathrm{~S}_{\mathrm{ij}}+\sum_{\mathrm{i}<j<k} \mathrm{~S}_{\mathrm{ijk}}+\mathrm{S}_{1,2, \ldots \mathrm{d}}=1$ 
hence:

$\mathrm{V}_{\mathrm{i}} / \mathrm{V}(\mathrm{Y})=\mathrm{S}_{\mathrm{i}}$ : the first order sensitivity indices

$\mathrm{V}_{\mathrm{ij}} / \mathrm{V}(\mathrm{Y})=\mathrm{S}_{\mathrm{ij}}$ : the second order sensitivity indices

$\mathrm{V}_{\mathrm{ijk}} / \mathrm{V}(\mathrm{Y})=\mathrm{S}_{\mathrm{ijk}}$ : the third order sensitivity indices.

The sensitivity indices have a natural interpretation since they represent the fraction of the total variance of $\boldsymbol{f}(\boldsymbol{X})$, which results from any individual factor or combination of factors. One limitation of Sobol's sensitivity indices is their high computational cost.

The first order sensitivity index $\left(\mathrm{S}_{\mathrm{i}}\right)$, which is called "main effect," represents the expected amount of variance removed from the total output variance, in case that the uncertainty of $X_{i}$ is known. This measure indicates the relative importance of an individual input variable $X_{i}$ in driving the uncertainty and can be seen as indicating where to direct effort to reduce that uncertainty. The first order indices are an essential parameter for the Factor Prioritization setting, which focuses on identifying the most important factor.

The total effect for the input variable $X_{i}$ is the sum of the first order index and all higher order effects in which the factor participates. The total index represents the expected amount of output variance that would remain if $X_{i}$ is left free to vary over its uncertainty range after all other variables have been fixed. The total sensitivity index can be expressed by:

$$
\mathrm{S}_{\mathrm{Ti}}=\frac{\mathrm{E}\left(\mathrm{V}\left(\mathrm{Y} \mid \mathrm{X}_{-\mathrm{i}}\right)\right]}{\mathrm{V}(\mathrm{Y})}
$$

where $\mathrm{X}_{-\mathrm{i}}$ represents all the input variables except $\mathrm{X}_{\mathrm{i}}$.

The total sensitivity index is the essential parameter for the Factors Fixing setting, which focuses on identifying the factor or the subset of input factors that can be fixed at any given value over their ranges of uncertainty without significantly reducing the output variance. If such a factor or subset of factors is identified, the remaining ones, being varied within their own range, explain most of the unconditional variance.

\subsubsection{General Analysis Procedure for Global Sensitivity Analyses and SimLab}

This study used SimLab, a global SA software designed for Monte Carlo-based uncertainty and SA (SimLab 2008). SimLab is based on performing multiple evaluations with probabilistically selected input factors and then using the results of these evaluations to determine the uncertainty in model predictions and the input factors that gave rise to this uncertainty (Satelli et al. 2004). In general, Monte Carlo-based analyses use the following five steps (Satelli et al. 2004):

1. Input Selection: A range and distribution are selected for each input variable (input factor). These selections will be used in the next step for the generation of a sample from the input factors. If the analysis is primarily of an extrapolatory nature, then a quite rough distribution assumption may be adequate.

2. Input Generation: A sample of points is generated from the distribution of the inputs specified in the first step. The result of this step is a sequence of sample elements.

3. Model Evaluation: The model is fed with the sample elements, and a set of model output is produced. In essence, these model evaluations create a mapping from the space of the input to the space of the results. This mapping is the basis for subsequence uncertainty and SA.

4. Uncertainty Analysis: The results of model evaluations are used as the basis for uncertainty analysis. One way to characterize the uncertainty is with a mean value and a variance. Other model output statistics are provided.

5. Sensitivity Analysis: The results of model evaluations are used as the basis for the SAs. 


\subsubsection{General Strengths and Weaknesses of Global Sensitivity Analyses}

It is quite useful to know the strengths and weaknesses of the Monte Carlo-based SA method before doing analyses. The typical strengths of Monte Carlo simulation are as follows:

- It provides comprehensive insight into how specified uncertainty in inputs propagate through a model

- It forces analysts to explicitly consider uncertainty and interdependencies among different inputs

- It is capable of coping with any conceivable shape of probability distribution function and can account for correlations

- It can be used in 2-D mode to separately assess variability and epistemological uncertainty.

The general weaknesses of Monte Carlo simulation are as follows:

- It is limited to those uncertainties that can be quantified and expressed as probabilities.

- One may not have any reasonable basis on which to ascribe a parameterized probability distribution to parameters.

- It may take a large run-time for computational intensive models. This can partly be remedied by using more efficient sampling techniques.

- The interpretation of a probability distribution of the model output by decision makers is not always straightforward. There is no single rule arising out of such a distribution that can guide decision-makers concerning the acceptable balance between, for instance, expected return and the variance of that return.

\subsection{Uncertainty and Sensitivity Analyses}

This section summarizes the uncertainty and SA on the PIV measurement error caused by oil temperature. This study uses global SA based on the random sampling Monte-carlo method. Figure 9 shows the procedure used.

Input Variables and Ranges

<SIMLAB 2.2 >

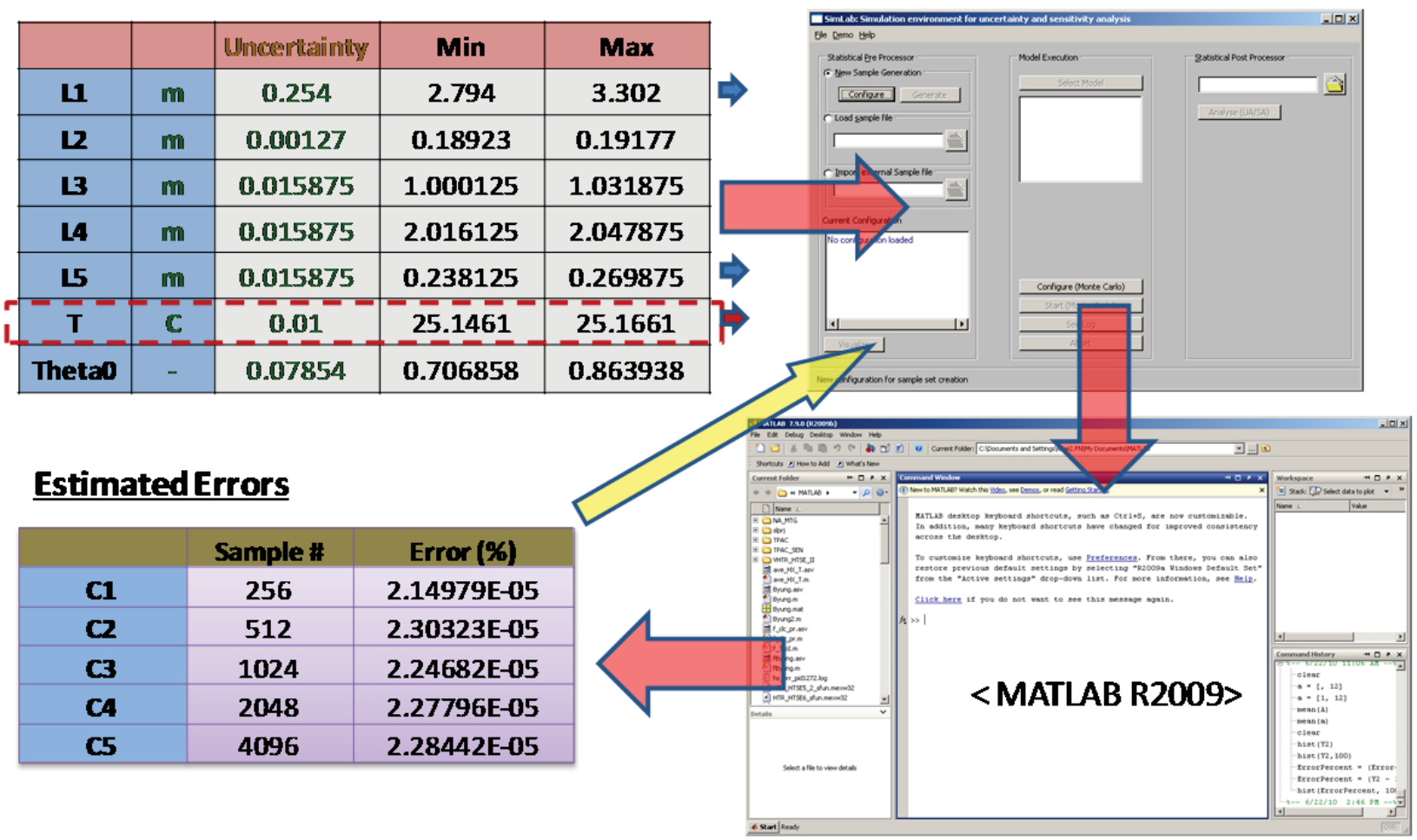

Figure 9. Analysis procedure. 
Seven input parameters considered important in image mapping were selected:

- L1 (m): distance in air between focus and glass

- L2 (m): distance between glass inside and outside

- $\quad$ L3 (m): distance in oil between glass and model wall (quartz)

- L4 (m): distance in quartz between model wall inside and outside

- L5 (m): distance in oil between model wall to measuring location

- $\mathrm{T}(\mathrm{C})$ : mineral oil temperature

- Theta0: initial camera view angle.

The parameter uncertainties were also predetermined in this step; their values are shown in Figure 9.

Based on the input parameters and uncertainties, input datasets were randomly generated using SimLab 2.2 software. SimLab 2.2 is a well-known SA code developed by the European Commission (SimLab 2002). This code can deal with various modern sampling based uncertainty/SA methods.

After the input datasets were generated, they were input into the MATLAB code developed for estimating velocity errors. The MATLAB code program was based on the method described in the previous sections. Appendix A shows the detailed MATLAB script.

After the outputs were generated by the MATLAB code, the results were re-input into the SimLab 2.2 software with input data. This process estimated average errors, standard deviations, and the importance of each parameter.

This analysis mainly focused on the effect of mineral oil temperature uncertainty between calibration and actual measurement conditions. However, in the actual measurement, the camera locations, camera angle, measurement locations, and model locations can deviate from calibration conditions other than oil temperature. These effects are discussed later in this document with some SAs.

Figure 10 shows a distribution of velocity errors for the input ranges given in Figure 9. In this analysis, temperature uncertainty was assumed to be $\pm 0.01^{\circ} \mathrm{C}$ during the measurement. As shown Figure 10, the velocity measurement error by oil temperature is very small, within $5 \times 10^{-5} \%$. It was estimated that average velocity measurement error was about $2.28 \times 10^{-5} \%$.

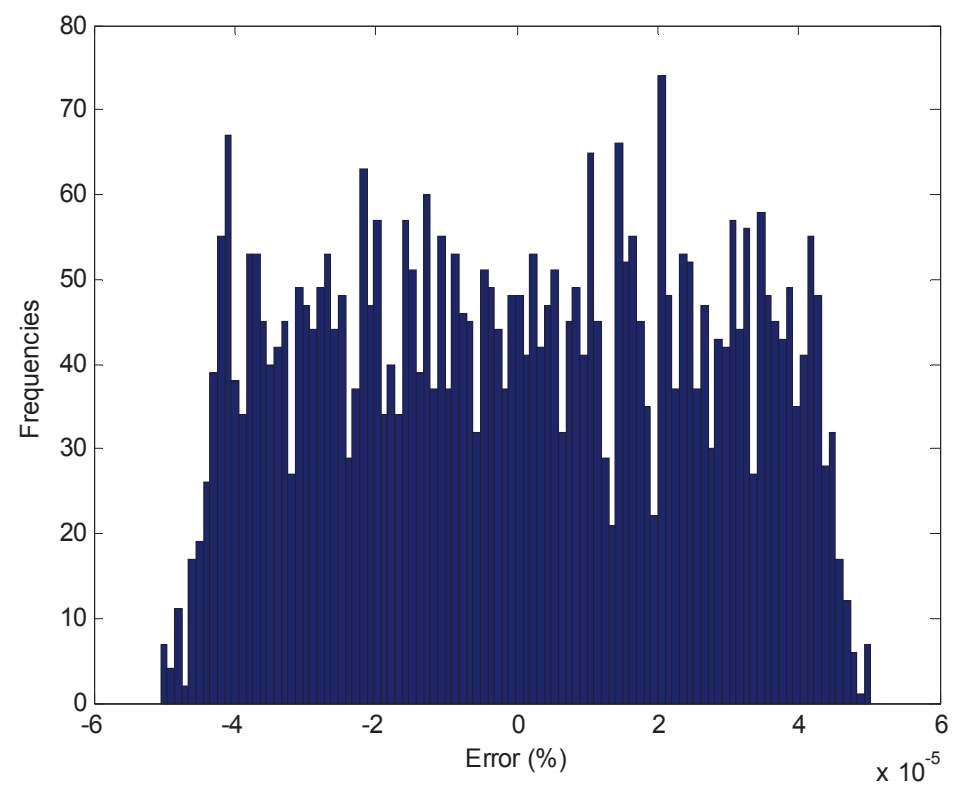

Figure 10. Estimated velocity measurement error from oil temperature. 
A parametric study of the velocity measurement error with mineral oil temperature uncertainty was conducted. Oil temperature uncertainty was changed from \pm 0.01 to $\pm 1.00^{\circ} \mathrm{C}$, as shown in Figure 11, where the velocity measurement uncertainty increased with temperature uncertainty, having a linear trend. For the temperature uncertainty of $\pm 1.00^{\circ} \mathrm{C}$, the velocity measurement error was estimated to be about $0.0025 \%$, indicating that the effect of oil temperature on the velocity measurement is negligible.

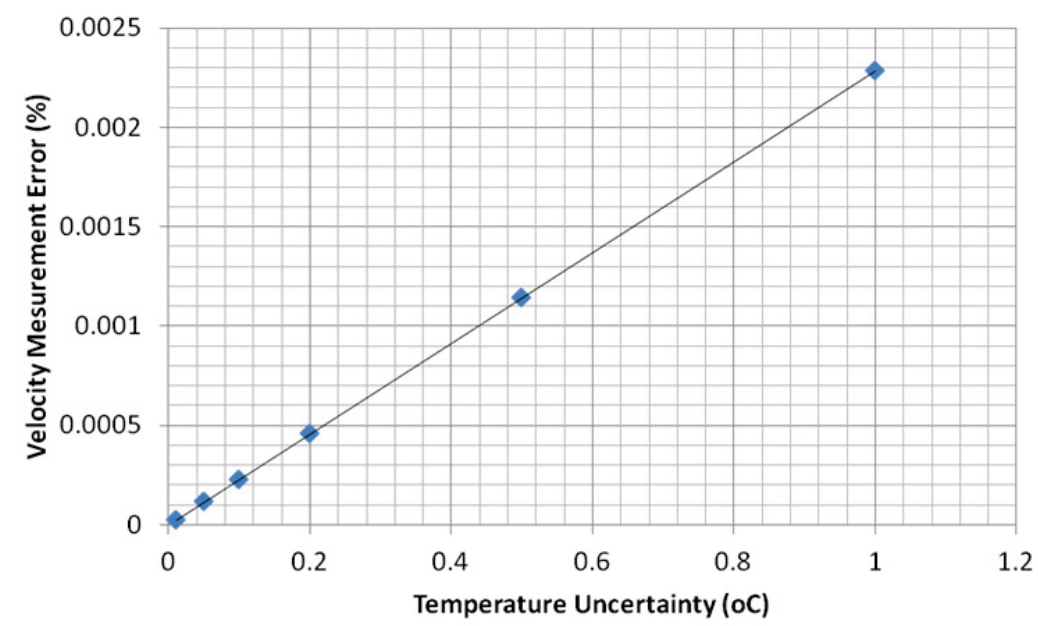

Figure 11. Effect of temperature uncertainty on velocity measurement error.

Based on the input uncertainties specified in Figure 11, this study conducted SA for velocity errors. All the input parameters were randomly generated in the input ranges and implemented into the MATLAB script for evaluations. The output results were processed in the SimLab software. Figure 12 shows the first order indices estimated by the Sobol method. The physical meaning of this sensitivity index is the contribution of output uncertainties by a certain parameter. The oil temperature in this study showed the largest sensitivity indices of 1.0, meaning that the oil temperature is the main uncertainty source of the velocity measurement error. Contributions of the other parameters were negligible compared to the oil temperature.

\begin{tabular}{|c|c|}
\hline Parameter & SI \\
\hline L1 & $\mathbf{0 . 0}$ \\
\hline L & 0.0 \\
\hline L3 & $\mathbf{0 . 0}$ \\
\hline L4 & $\mathbf{0 . 0}$ \\
\hline L5 & $\mathbf{0 . 0}$ \\
\hline T & 1.0 \\
\hline Theta0 & $\mathbf{0 . 0}$ \\
\hline
\end{tabular}

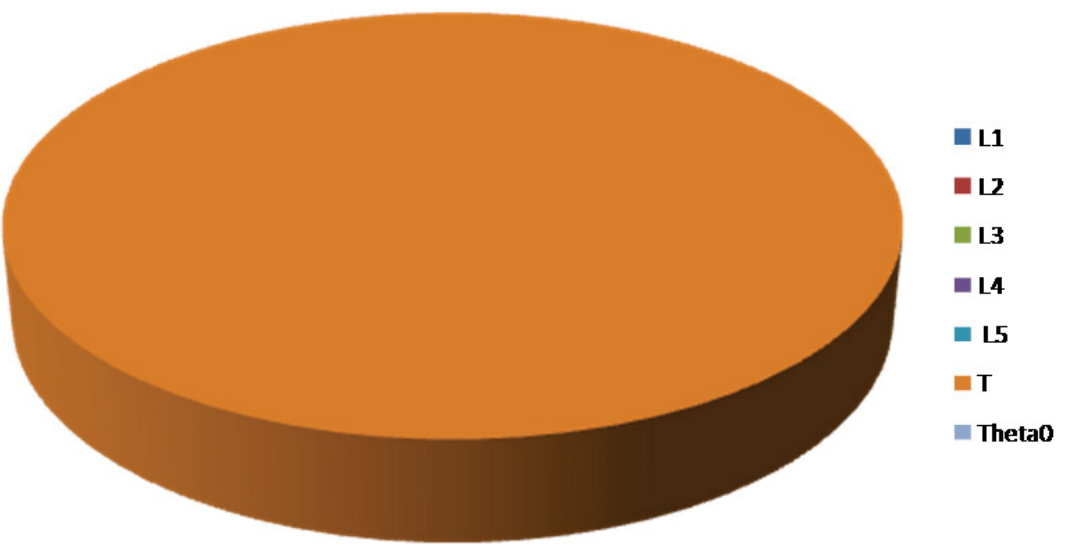

Figure 12. First order sensitivity index (Main Effect). 


\subsection{Summary}

This study investigated optical uncertainties related to the PIV measurement in the MIR facility. The measurement error quantification method was developed based on the mapping process from target object to the image planes. Uncertainty and SAs proposed by Sobol et al. (1990) were used to estimate total velocity measurement uncertainties and the importance of related parameters. This method was based on the random sampling Monte-carlo technique. The effect of oil temperature was estimated to be around $2.28 \times 10^{-5} \%$ for $\pm 0.01{ }^{\circ} \mathrm{C}$ and $0.0025 \%$ for $\pm 1.00^{\circ} \mathrm{C}$. According to SAs results, the contribution of the oil temperature on the velocity measurement error was estimated to be almost $100 \%$ of the total uncertainty. It was concluded that the effect of oil temperature is dominant. 


\section{EXPERIMENTS AT MIR FLOW FACILITY}

The Bypass Flow Experiment was performed in the MIR facility in which PIV measurements of a representative gas-cooled prismatic reactor core model were taken. These measurements were taken in order to understand the amount of flow that travels through the interstitial region between the fuel blocks and to produce benchmark data for CFD model validation.

The Flat Plate experiment within the MIR facility was designed and analyzed to use PIV to measure the flow within the bypass transitional flow regime. These measurements were used to better understand the entropy generation rate within this region of flow. Secondly, it was proposed that the entropy generation rate could be used as a design parameter to predict the onset of turbulence. Both mentioned experiments are discussed in further detail in the following sections.

\subsection{Bypass Flow Measurements in a Representative Gas-Cooled Prismatic Reactor Core Model}

Bypass flow is a unique characteristic of the Gas Turbine-Modular Helium Reactor (GT-MHR) designed by General Atomics. For a prismatic-type core, it refers to the coolant that navigates through the interstitial passages between the fuel elements and reflector regions in lieu of traveling through the designated coolant channels. It is estimated that bypass flow will vary from $10-25 \%$ or more of the total core flow and may also vary over time because of fast neutron-induced graphite element shrinkage and core barrel swelling (INEEL 2005). These flows are of particular concern because they reduce the desired flow rates in the coolant channels and thereby may have significant influence on the maximum fuel element temperature, cooling channel exit temperature, and the temperature distribution in the lower plenum; all of which affect the efficiency of the reactor. Bypass flow is considered the most important contributor to uncertainty in fuel temperatures (INL 2010).

\subsubsection{Prismatic Fuel Block Design}

General Atomics designed the GT-MHR with a prismatic-type core that uses helium as its coolant (General Atomics 1996). This type of core consists of a large group of tightly stacked hexagonal graphite prismatic fuel elements. The blocks are arranged in the reactor vessel with inner and outer graphite reflector regions that surround an annular core of fuel blocks, as shown in Figure 13.

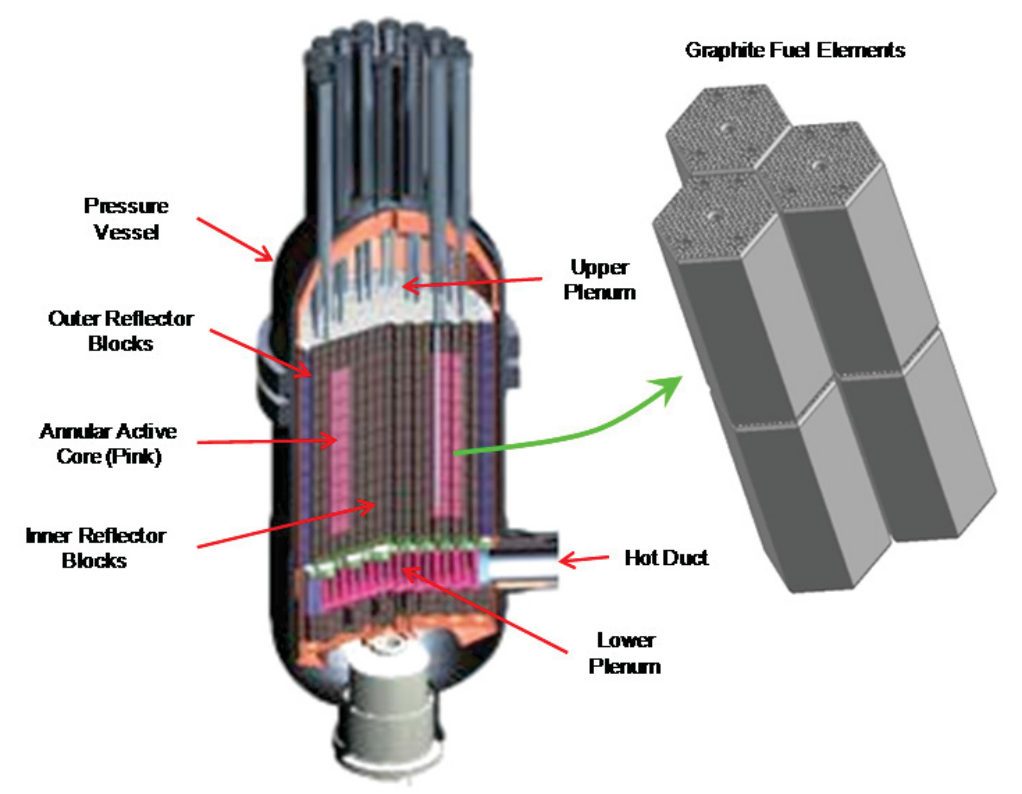

Figure 13. Schematic of the GT-MHR. 
During operation, cold helium flows from the outer annulus of the hot duct, up the walls of the pressure vessel, and into the upper plenum. The coolant then travels downward through a series of stacked graphite fuel blocks that contain an array of coolant channels. The mass flow rate for the helium is expected to reach $320 \mathrm{~kg} / \mathrm{s}$ at full power. During normal operation, the coolant channel outlet Reynolds number varies from approximately 57,000 at high power to 2,300 at $10 \%$ core power. Table 3 contains select specifications for the GT-MHR.

Table 3. Selected GT-MHR design parameters (General Atomics 1996).

\begin{tabular}{|lc|}
\hline \multicolumn{1}{|c|}{ Specification } & Value \\
\hline Core Power (MW) & 600 \\
Helium Mass Flow Rate (kg/s) & 320 \\
Active Core Pressure Drop (MPa) & 0.051 \\
\hline
\end{tabular}

\subsubsection{Fuel Element Geometry}

Active core fuel elements are manufactured from graphite in the form of right hexagonal prisms. Each element measures 793-mm high by 360-mm across, as shown in Figure 14. An array of fuel holes and coolant channels, with diameters of 12.7 and $15.88 \mathrm{~mm}$, respectively, run parallel through the length of each prism in a regular triangular pattern. At the center of each block is a fuel handling hole surrounded by six small coolant channels with diameters of $12.7 \mathrm{~mm}$.

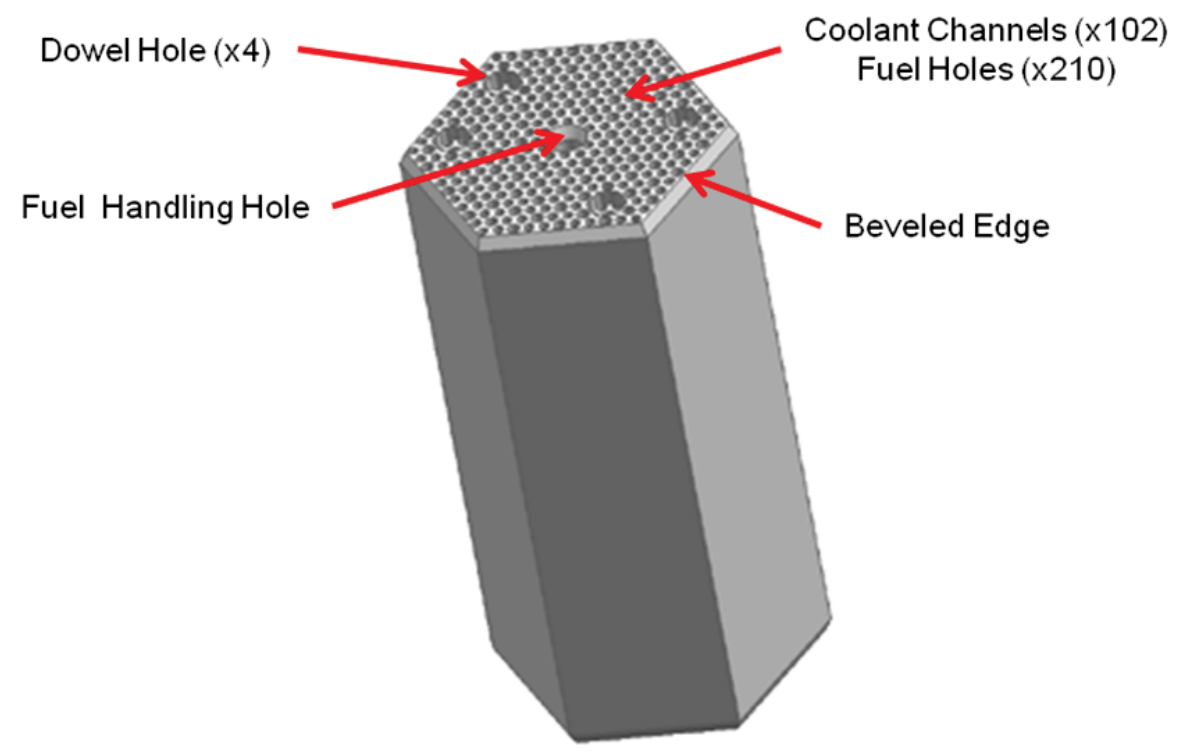

Figure 14. Standard fuel element.

The blocks are primarily aligned using a dowel-socket connection with its upper and lower neighbors, which are designed to fit together closely. The top and bottom edges of each element are beveled, with dimensions of 17.145 and $8.304 \mathrm{~mm}$. The bevel is used to assist insertion of the elements into the core. Table 4 lists pertinent fuel element parameters for the present study.

The gaps between fuel elements have a maximum width of approximately $3.9 \mathrm{~mm}$ upon installation. Though the fuel elements are designed to pack the core tightly, it is known that they will change size over their lifetime (Melese and Katz 1984). 
Table 4. Pertinent fuel element parameters (General Atomics 1996).

\begin{tabular}{|ll|}
\hline \multicolumn{1}{|c|}{ Parameter } & \multicolumn{1}{c|}{ Value } \\
\hline Distance Across Flats $(\mathrm{m})$ & 0.36 \\
Element Height (m) & 0.7930 \\
Coolant Holes per Element, Small/Large & $6 / 102$ \\
Coolant Hole Diameter, Small/Large (mm) & $12.7 / 15.88$ \\
Fuel Holes per Element & 210 \\
Fuel Hole Diameter (mm) & 12.7 \\
Control Rod Hole Diameter (mm) & 101.6 \\
Triangular Pitch Between Coolant Holes (mm) & 18.796 \\
Beveled Edge Height/Width (mm) & $17.145 / 8.304$ \\
\hline
\end{tabular}

Graphite expands as temperature increases and is expected to shrink slightly because of irradiation. Melese et al. (1984) estimates a decrease of $5.1 \mathrm{~mm}$ in height and $3.5 \mathrm{~mm}$ across the flats over a 4-year lifespan. Since neither temperature nor irradiation is uniform, these effects will modify the shape of the elements and unevenly influence the gap size between adjacent blocks. The spacing between each block will vary, depending on such graphite shrinkage, manufacturing tolerances, and inexact installation. These gaps between the fuel elements allow for the helium coolant to travel in two general flow paths: axially (parallel to the coolant channels) and radially (in between the stacked fuel blocks).

\subsubsection{Bypass Flow Literature Review}

Several studies conducted in the 1980s presented 1-D nodal and analytical methods to investigate bypass flow (Kaburaki and Takizuka 1987); however, these methods were quite simplistic. In recent years, studies have focused on CFD calculations performed by researchers from INL, Japan Atomic Energy Research Institute, and Korea Atomic Energy Research Institute. Since only limited experimental studies focus on bypass flow, accurate fluid dynamic experiments must be performed to validate CFD-based research and predictions.

Sato, Johnson, and Schultz (2010) performed 3-D CFD calculations of a typical 600 MW very high temperature reactor core. Their model represented a one-twelfth sector of a hexagonal fuel block, which was selected to achieve the smallest possible symmetric slice. The simulation considered an inlet temperature of $490^{\circ} \mathrm{C}$, a mass flow rate of $0.2 \mathrm{~kg} / \mathrm{s}$, and a heat generation rate of $27.88 \mathrm{MW} / \mathrm{m}^{3}$ with uniform gaps. The region between the fuel blocks was varied for each simulation using 0,3 , and 5-mm spacings. This variation in gap width caused the channel Reynolds number to range between 20,000 and 35,000 for the largest and smallest gap, respectively. The results indicated that the gap to coolant channel flow fraction increased from 4.15 to $9.78 \%$, considering a gap width increase from 3 to $5 \mathrm{~mm}$. As the gap size increased, the maximum fuel temperature increased by $62^{\circ} \mathrm{C}$, the temperature difference variation between coolant channels increased by $101^{\circ} \mathrm{C}$, and the maximum channel outlet temperature decreased by $55^{\circ} \mathrm{C}$. These changes established a large lateral thermal gradient across the sector and increased the possibility of hot streaking in the lower plenum.

Yoon et al. (2007) studied the bypass flow of a prismatic modular reactor design using both experimental and computational methods. Their model introduced the concept of a unit-cell, which included three sectors, each made from a one-sixth part of a prismatic fuel block, separated by a uniform gap. The coolant channels for each sector were represented by a single $0.12-\mathrm{m}$ tube, equivalent to the total flow area. The total unit-cell area made up one-half of a prismatic fuel block. The gaps were varied by 3 and $5 \mathrm{~mm}$. Using air at $25^{\circ} \mathrm{C}$, the inlet flow rate was varied to achieve approximately $0.18,0.37$, and $0.56 \mathrm{~kg} / \mathrm{second}$, with estimated channel Reynolds numbers ranging from 32,000 to 99,000. Using these inlet conditions, it was learned that the bypass ratio varied from 2.48 to $5.70 \%$ for the $3-\mathrm{mm}$ gap and 7.52 to $11.97 \%$ for the 5-mm gap. Fluid dynamic computations were performed for the 5-mm case using the shear stress transport turbulence model with 2.9 million elements; however, differences as high as $27 \%$ were calculated from the 
experimental results. Other model combinations for bypass flow around a reflector block were studied, but those cases are not considered here.

Pretest CFD calculations for the present bypass flow experiment were carried out at INL by Johnson (2011). The geometry was nearly identical to the present study, with the exception that symmetry was applied and a one-sixth sector was selected; the model was composed of 1.5 coolant channels and half a gap width. The coolant path consisted of an upper plenum, a full-length fuel element, and then a partial-length fuel element. This geometry allowed for both axial and radial gaps. The axial gaps varied in size by 2,6 , and $10 \mathrm{~mm}$ and the radial gaps varied by 2 and $10 \mathrm{~mm}$. Mineral oil was selected as the working fluid at $25^{\circ} \mathrm{C}$, and volumetric flow rates of 205.3 and $57.2 \mathrm{~L} / \mathrm{min}$ were used. The $\mathrm{k}-\varepsilon$ turbulence model was employed with near wall treatment, and the solution was iterated to residuals $<1 \times 10^{-4}$.

Table 5 summarizes the results, which show that:

- The bypass flow fraction in the upper block was higher than the lower block for all cases

- Flow in the gap increased with increasing gap size

- The bypass flow fraction in the 6-mm axial gap was similar for both the low- and high-flow rate cases, even though one was laminar and one was turbulent

- Flow in the channels was turbulent in all cases

- Flow in the gap was laminar in all cases, except the high-flow rate 10-mm axial gap scenario.

Table 5. Summary of CFD calculations for one-sixth sector of the bypass model (Johnson 2011).

\begin{tabular}{|c|c|c|c|c|c|c|}
\hline Axial Gap (mm) & & & & 6 & & 10 \\
\hline Radial Gap (mm) & 2 & 10 & 2 & 10 & 2 & 2 \\
\hline Flow Rate (L/min) & 205.3 & 205.3 & 205.3 & 205.3 & 578.2 & 578.2 \\
\hline $\operatorname{Re}_{\text {Channel }}$ & 6,387 & 6,387 & 5,440 & 5,400 & 15,024 & 12,362 \\
\hline $\operatorname{Re}_{\text {Gap }}$ & 31 & 32 & 524 & 543 & 1,668 & 3,192 \\
\hline Pressure Drop $(\mathrm{kPa})$ & 13.5 & 13.7 & 10.4 & 10.6 & 64.7 & 45.3 \\
\hline Bypass Flow Upper Block (\%) & 0.97 & 0.97 & 15.6 & 16.2 & 17.3 & 31.9 \\
\hline Bypass Flow Lower Block (\%) & 0.61 & 0.65 & 13.4 & 12.4 & 14.9 & 29.9 \\
\hline
\end{tabular}

\subsubsection{Bypass Flow Experiment}

An experimental study at INL investigated the behavior of flow in the interstitial regions between fuel blocks of a very high temperature reactor design, particularly to quantify the flow through the gap in relation to the coolant channels. This study was carried out in the MIR facility where PIV was used to measure the flow field within a scaled model. A simplified model of the GT-MHR was considered, wherein a stacked junction of six adjacent fuel blocks were represented. Fuel block geometry was scaled by a factor of 2.016 to geometrically match the prismatic core configuration. Figure 15 illustrates the cross-section of the bypass model and the vertex of the three adjoining fuel blocks from which it was derived. As shown, a small gap existed between the blocks, depicting the interstitial gap region. The cross-section of the model consisted of three sectors of intersecting prismatic fuel blocks, which included nine coolant channels and three axial gaps. Each fuel block was carefully sectioned to only include the area that reasonably influenced the flow into the coolant channels and interstitial gaps. 


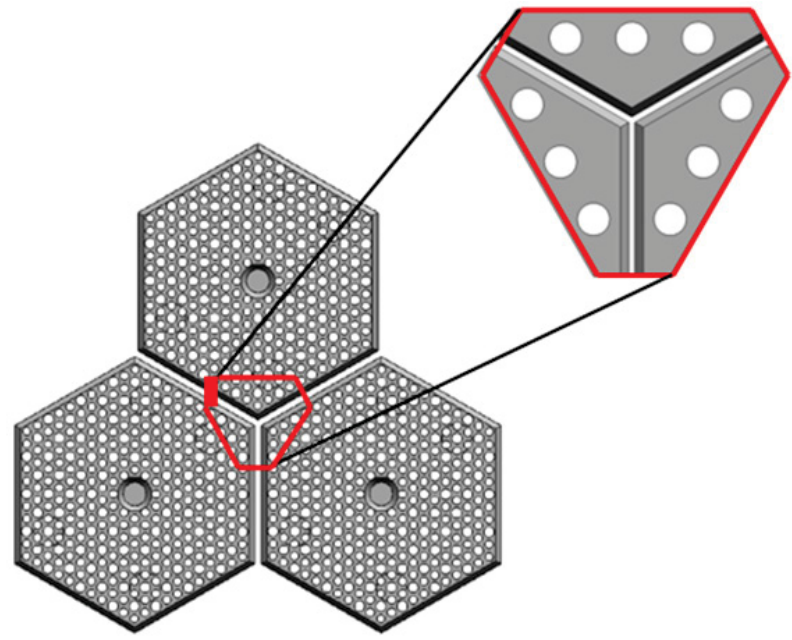

Figure 15. Bypass model relationship to GT-MHR.

The scaling factor was selected based on the commercially available diameter quartz tubes, which were used as the coolant channels for the model to permit Reynolds numbers comparable to the low-power range of an operational prismatic reactor. Careful selection of the scaling factor was also important because it was the key feature to provide adequate spatial resolution for SPIV measurements in the coolant channels and gaps. Based on this criterion, a tube diameter of $32 \mathrm{~mm}$ was chosen.

The experimental apparatus included two near double-scale blocks: a full-length upper block and a 20\% partial-length lower block. Each sector was manufactured from three parallel tubes that connected to a solid, machined cap on each end. The caps were chamfered to match those of a typical fuel block. Long plates extended between the caps to serve as walls for the interstitial gaps. All quartz components were manufactured to a $0.127-\mathrm{mm}$ tolerance. The gaps between sectors were adjustable to widths of approximately 2, 6, and $10 \mathrm{~mm}$ using a spacer that runs along the length of the model. Epoxy adhesive and Room Temperature Vulcanizing silicone were used to bond and seal the model, but optical glue was used to seal the joints where optical or laser access was required.

The model was constructed from fused quartz to permit optical access for PIV measurements. Quartz was used because its refractive index is similar to mineral oil - the working fluid in the MIR facility. By pairing these media at their index matching temperature of $25.156^{\circ} \mathrm{C}$, the model became near invisible when submersed in the mineral oil, and optical refraction was eliminated between the wall of the test section and the inside of the model. This technique is advantageous to measure internal flow.

Figure 16 depicts the components of the bypass flow model. Flow entered the model through the inlet annulus, where it was directed to the periphery of a hemisphere. The hemisphere contained an anti-separation spike that prohibited the flow from separating as it turned 180 degrees to travel through the upper plenum and representative fuel blocks. 


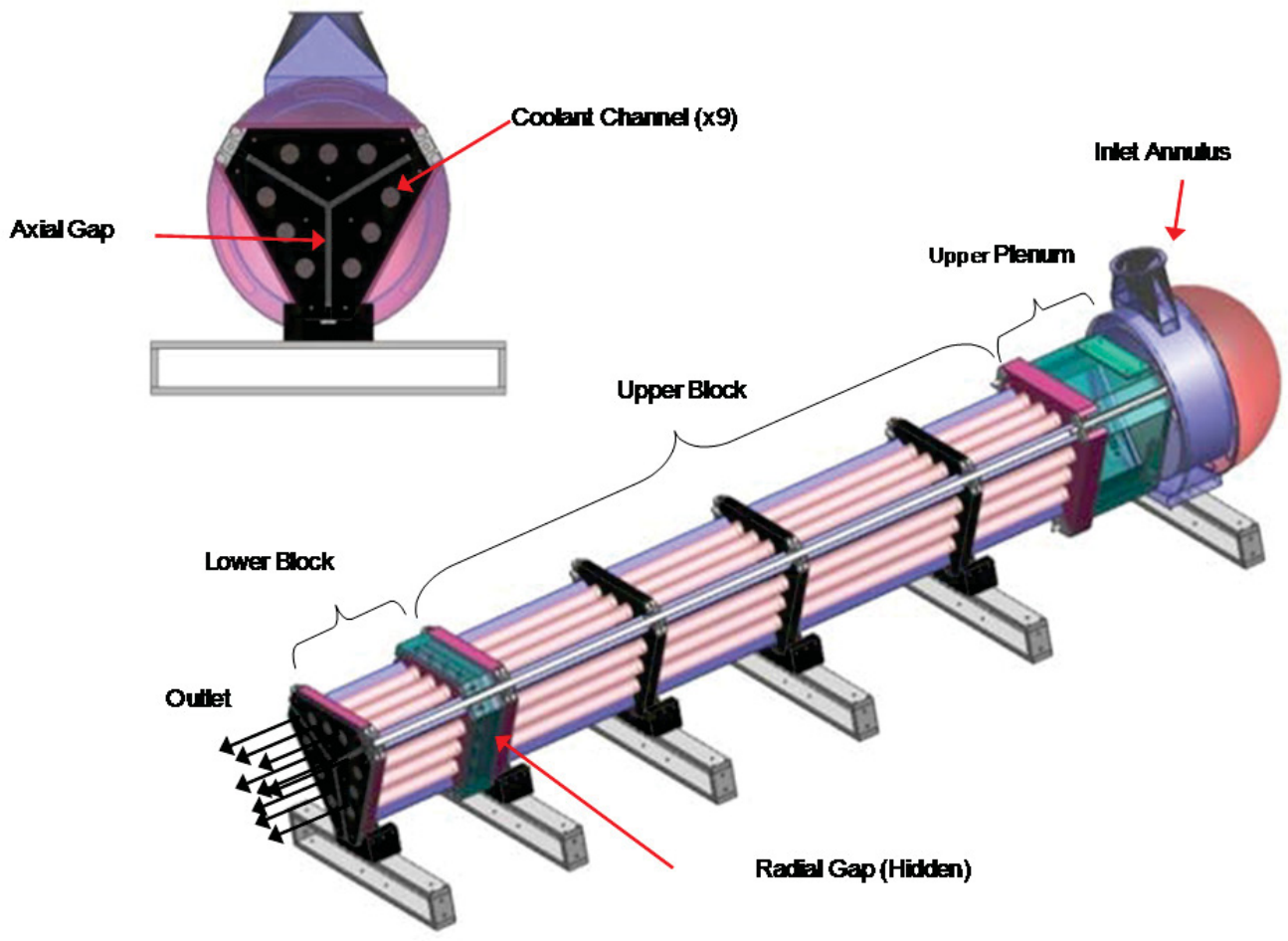

Figure 16. Bypass model components.

A pump analysis of the total dynamic head for the bypass flow model was performed to estimate the Reynolds numbers expected in the coolant channels and gaps given the MIR system; specifically, an auxiliary loop pump provided flow into the model, MIR plumbing, and bypass model geometry. Figure 17a illustrates the auxiliary loop pump curve and estimated head loss with varying flow rate. Figure $17 \mathrm{~b}$ shows expected Reynolds numbers in the coolant channels and gaps for varying flow rates. It was determined that a maximum flow rate of $1,430 \mathrm{~L} / \mathrm{min}$ could be achieved given a $3-\mathrm{mm}$ gap width, leading to channel and gap Reynolds numbers of 7,282 and 360, respectively. This maximum assumes little or no leakage from the model.

The gap width was used as the characteristic length to calculate the Reynolds number in the gap, allowing direct comparison to the CFD pre-calculations performed by Johnson.

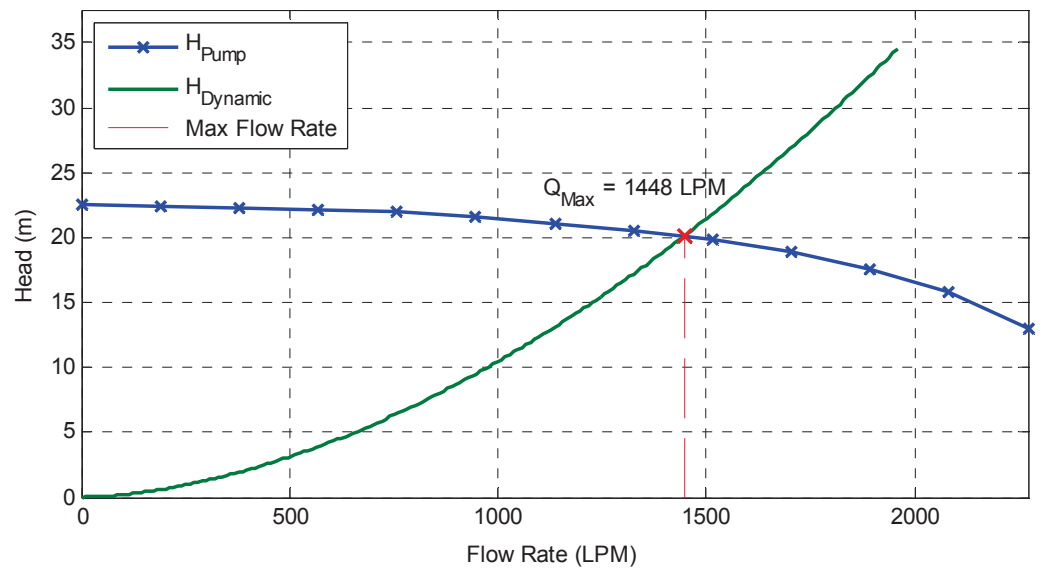

Figure 17a. Head loss calculations. 


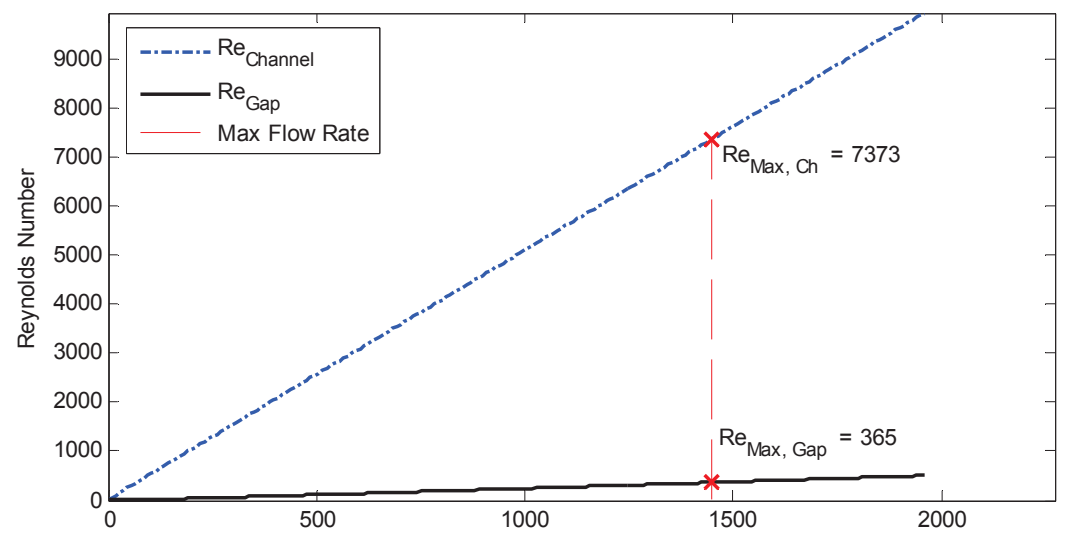

Figure 17b. Design head loss and Reynolds number estimates.

Because the MIR is an isothermal facility, the effects of density gradients and the influence of temperature will not be studied. The present experiment will focus on the flow regimes that are dominated by momentum as opposed to density gradient.

\subsubsection{Test Plan}

SPIV was used to capture the flow field within the upper plenum, coolant channels, and gaps. The flow was seeded with $12-\mu \mathrm{m}$-diameter, silver-coated, hollow-glass spheres, which illuminated in 532-nm laser light. A neodymium-doped yttrium aluminum garnet double-pulsed laser projected a light sheet upward through the bottom of the model in the stream-wise direction, and two special charged coupled device cameras continuously imaged the seeding particle movement. Sequential images were broken down into IWs, and statistical algorithms were employed to determine particle movement, after which velocity vectors were calculated over the FOV. A sample size of 500 vector maps was used to ensemble averages for each flow field measurement.

Measurements were taken in three stream-wise locations: the upper plenum, the midsection of the upper fuel blocks, and the midsection of the lower fuel blocks as shown in Figure 18. In these locations, the laser light sheet and cameras were translated across the width of the model, and velocity fields were measured at millimeter intervals - 301, 245, and 245 slices were taken in the upper plenum, upper block, and lower block, respectively. As a note, the model's origin was located at the centerline of the model and the inlet of the upper plenum.

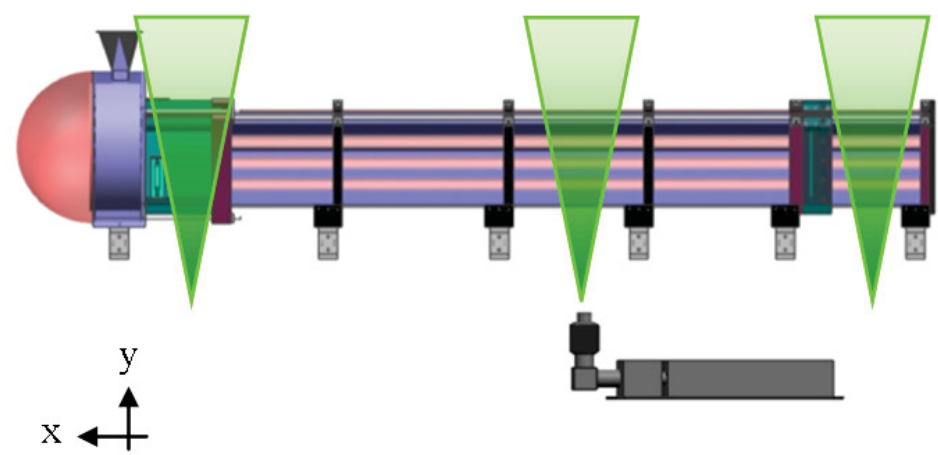

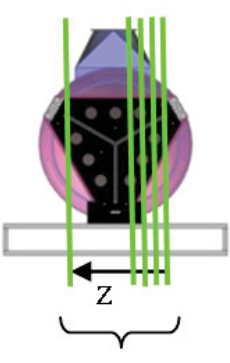

Light Sheets (1mm Intervals)

Figure 18. Light sheet locations.

Table 6 contains the data collection matrix for the present experiment. Inlet conditions were varied to incorporate laminar, transitional, and turbulent flows in the coolant channels. For the contents of this paper, the axial and radial gaps were held constant using nominal values of 6 and $2 \mathrm{~mm}$, respectively. 
Table 6. Data collection matrix for the present experiment.

\begin{tabular}{|llcccc|}
\hline & & & \multicolumn{2}{c|}{ Light Sheet Positions in 1 mm Intervals } \\
\cline { 3 - 6 } ReCh & $\begin{array}{c}\text { Axial Gap } \\
(\mathrm{mm})\end{array}$ & $\begin{array}{c}\text { Radial Gap } \\
(\mathrm{mm})\end{array}$ & $\begin{array}{c}\text { Upper Plenum } \\
(\mathrm{mm})\end{array}$ & $\begin{array}{c}\text { Upper Block } \\
(\mathrm{mm})\end{array}$ & $\begin{array}{c}\text { Lower Block } \\
(\mathrm{mm})\end{array}$ \\
\hline 1,700 & 6.05 & 2.02 & $-152<\mathrm{z}<+152$ & $-122<\mathrm{z}<+122$ & $-122<\mathrm{z}<+122$ \\
3,000 & 6.05 & 2.02 & $-152<\mathrm{z}<+152$ & $-122<\mathrm{z}<+122$ & $-122<\mathrm{z}<+122$ \\
4,800 & 6.05 & 2.02 & $-152<\mathrm{z}<+152$ & $-122<\mathrm{z}<+122$ & $-122<\mathrm{z}<+122$ \\
\hline
\end{tabular}

During model assembly, spacers were used to achieve uniform axial gaps; however, after installation in the test section, the interstitial gap width was measured and found to vary between 5.47 and $7.75 \mathrm{~mm}$. Figure 19 shows the actual widths of the interstitial gap for the cross-section of the bypass model measured at the inlet of the upper block. The assembled gap had a cross-sectional area that was approximately $0.0028 \mathrm{~m}^{2}$.

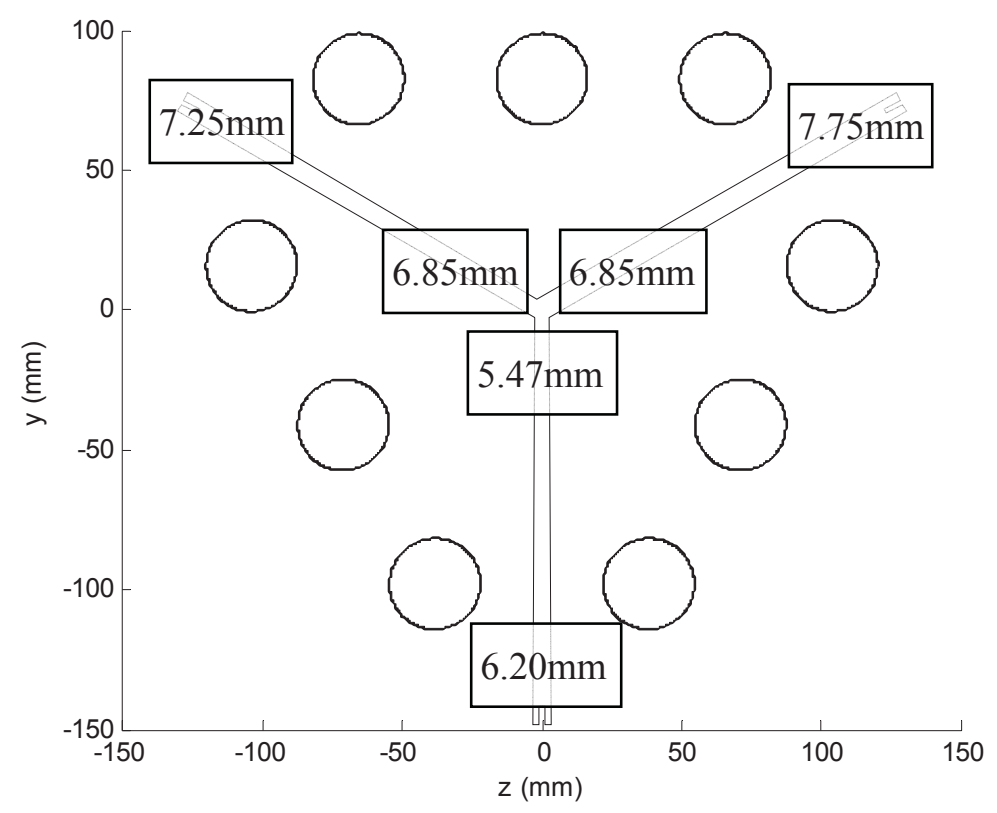

Figure 19. Actual gap spacing of the model cross-section.

\subsubsection{Experimental Methods}

The model was installed and aligned in the MIR test section within $0.076 \mathrm{~mm}$ of being level, plumb, and square to the traversing system - the apparatus used to position the cameras. A flow rate of $0.15 \mathrm{~m} /$ second was used to maintain the temperature within $\pm 0.005^{\circ} \mathrm{C}$ of the matching temperature. The flow rate into the model was adjusted to approximate the desired Reynolds numbers from the test matrix.

Velocity measurements were realized using commercially available PIV software with twin, double-pulsed $532 \mathrm{~nm}$ neodymium-doped yttrium aluminum garnet lasers. Two 1.9 MPa cameras focused on the model through 50-mm lenses at acute angles (less than 15 degrees) to the test section windows. Small angles were desired to minimize refraction at the test section air-to-window interface. Scheimpflug adapters were installed to give a sharp focus over the entire FOV.

The cameras were calibrated using a split level $309 \times 309-\mathrm{mm}$ plate that filled the entire FOV. The plate was manufactured with an array of 3-mm dots that were $15-\mathrm{mm}$ apart and 3-mm between levels. A polynomial model was applied to map the FOV, then self-calibration was performed to correct any misalignment of the laser light sheet. The light sheet was adjusted to a thickness of $1 \mathrm{~mm}$, allowing for complete coverage of the model within the limits of the test matrix. 
An IW of $12 \times 12$ pixels was chosen for processing, and flow was seeded such that at least 8 to 10 particle pairs were in each window. The cameras collected data at a frame rate of $15 \mathrm{~Hz}$. The time delay between sequential frames was chosen based on the criteria set by Wilson and Smith (2011). Particle displacements were limited to traveling between 25 and $62.5 \%$ of the IW.

Images were processed first with a $16 \times 16$ IW size and then with a $12 \times 12$ window size, using $50 \%$ overlap for both. The IW was selected such that the gap, the smallest feature size, had a minimum of 4 to 5 vectors after processing.

A General Public Utilities-based Personal Computer with 580 cores processed the data using the direct cross-correlation method. Because of storage and processing requirements, each span-wise velocity measurement was stored on a single 2-terabyte removable hard drive. The prescribed test matrix produced about 18 terabytes of data, taking over three months to process.

\subsubsection{PIV Sample Size Analysis}

An analysis similar to that of Uzol and Camci (2001) helped estimate the sample size N (number of vectors maps) that needed to be averaged to calculate stable vector and turbulence quantities, which were independent of time. The analysis was conducted using 4,000 instantaneous vector maps for a typical slice of the flow at a $600 \mathrm{~L} / \mathrm{min}$ flow rate $(\mathrm{ReCh} \approx 2800)$.

At a specific location in the FOV, sets of $\mathrm{N}$ instantaneous vectors were randomly selected from the available 4,000, averaged, and plotted in relation to the total mean. This process was repeated 100 times for each $\mathrm{N}$ to statistically depict how the spread in the average decreased with increasing sample size. The $\mathrm{N}$ was varied between 5 and 4,000 samples. It was noted that in calculating the ensemble-average for the flow field, some regions of the FOV converged slower than others (required more images). Hence, the slow convergent regions dictated the number of images required to calculate an accurate ensemble-average. The above mentioned process was completed over an entire FOV (containing channel and gap flow) to determine the location of slowest convergence. Figure 20 illustrates the convergence at a typical point in the flow for mean velocity and turbulent variation with increasing sample size. It was determined that 500 samples could adequately represent velocity and turbulence quantities given the space requirement and computational cost of the test matrix.
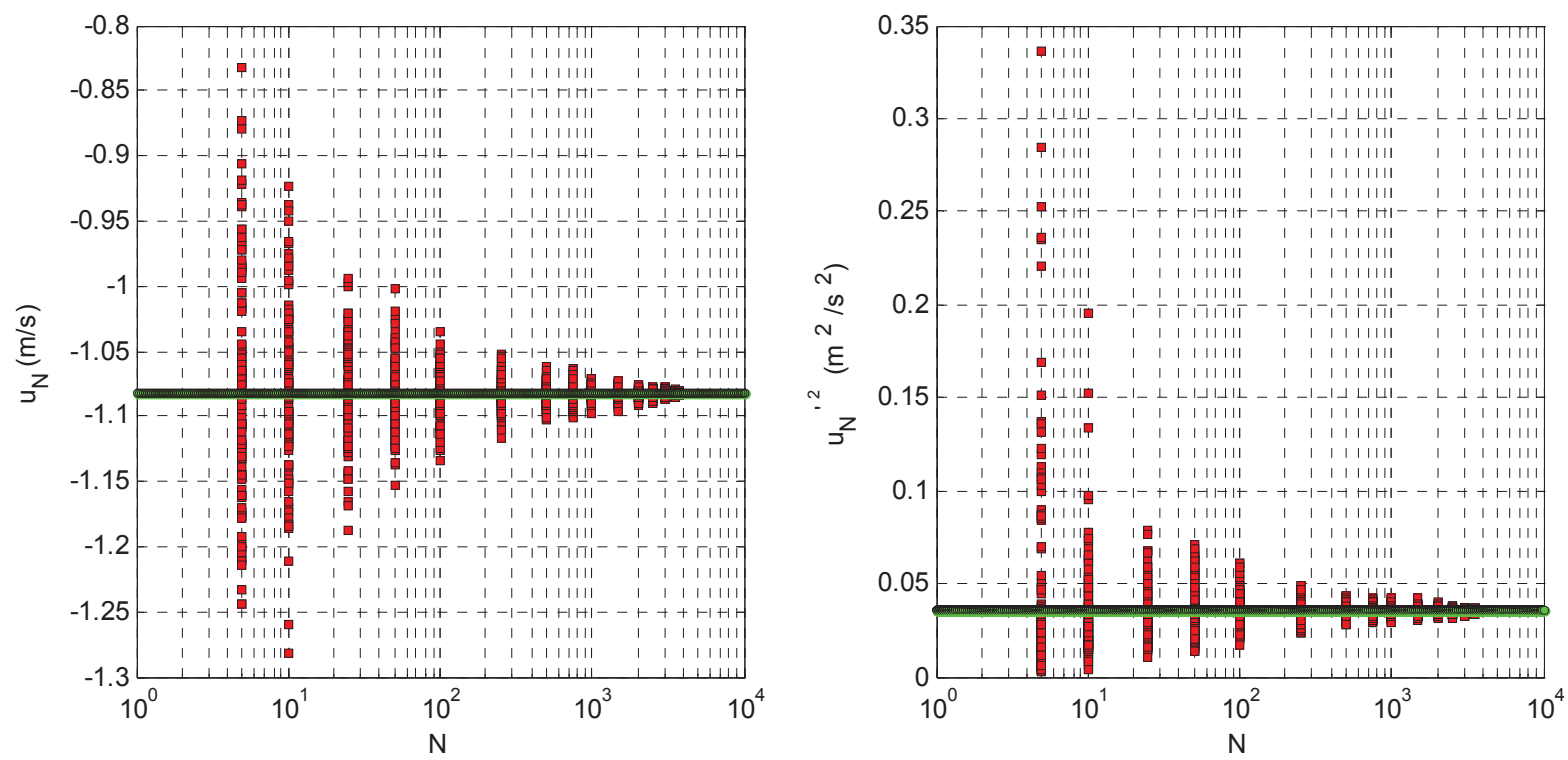

Figure 20. Variation in (a) mean velocity and (b) mean turbulence stress versus sample size. 


\subsubsection{Flow Rate Analysis}

To quantify the flow of the gap in relation to the coolant channels, velocities from the cross-section of the model were needed. To this end, time averaged vector maps at each stream-wise location and flow rate were compiled into matrices, after which data slices were taken at three locations perpendicular to the flow at $x=-113.1 \mathrm{~mm},-1029.5 \mathrm{~mm},-1928.5 \mathrm{~mm}$ as shown in Figure 21. For the purpose of this paper, the measurements taken in the upper plenum were used solely as a flow rate check.

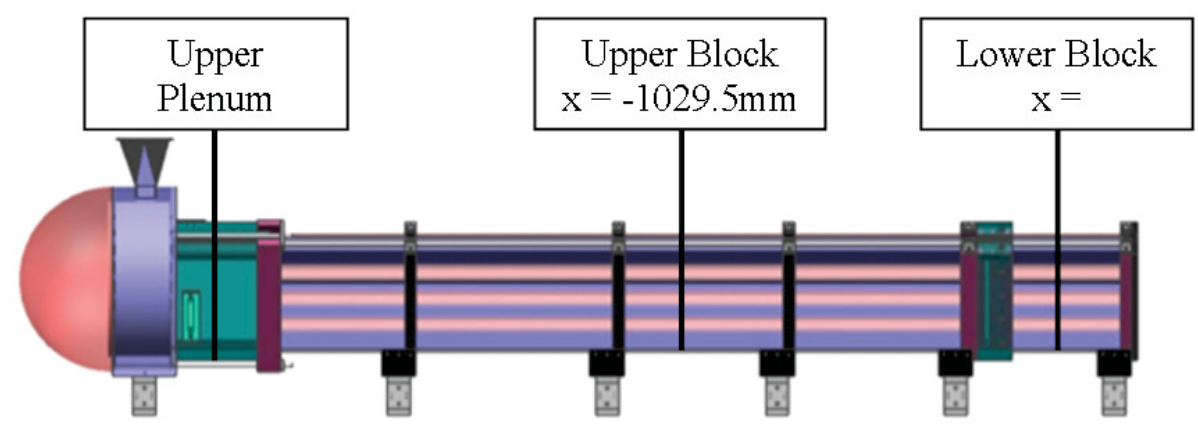

Figure 21. Data slice locations.

A typical slice of the bypass data located in the upper and lower blocks is displayed in Figure 22. This plot denotes the velocity contours through the cross-section of the model. Note that the PIV cameras had limited optical access to the upper three coolant channels. As such, the PIV algorithms did not detect movement of particles and effectively calculated streaks of zero velocity in these locations.

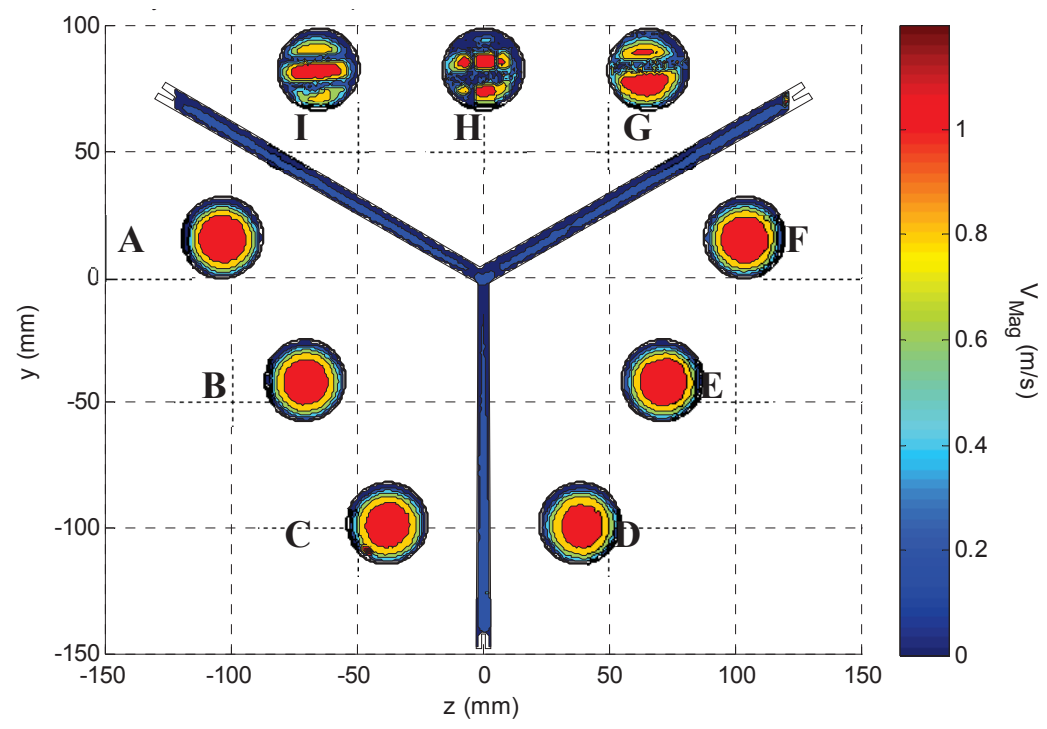

Figure 22. Typical velocity contour plot $(\mathrm{Q}=351.15 \mathrm{~L} / \mathrm{min}$ and $\mathrm{x}=-1029.5 \mathrm{~mm})$.

The commercial software MATLAB was used to compile the vector maps because of its ability to manipulate large matrices. A geometric mask was applied to the data to disregard vectors outside the flow area. The remaining zones were subdivided into small rectangular regions, sized by the light sheet spacing (dz) and IW height (dy); the area of which was defined by $d A=d z \cdot d y$. Figure 23 illustrates the typical flow area discretization within a coolant channel. Flow in the gaps and upper plenum were calculated in a similar fashion. 


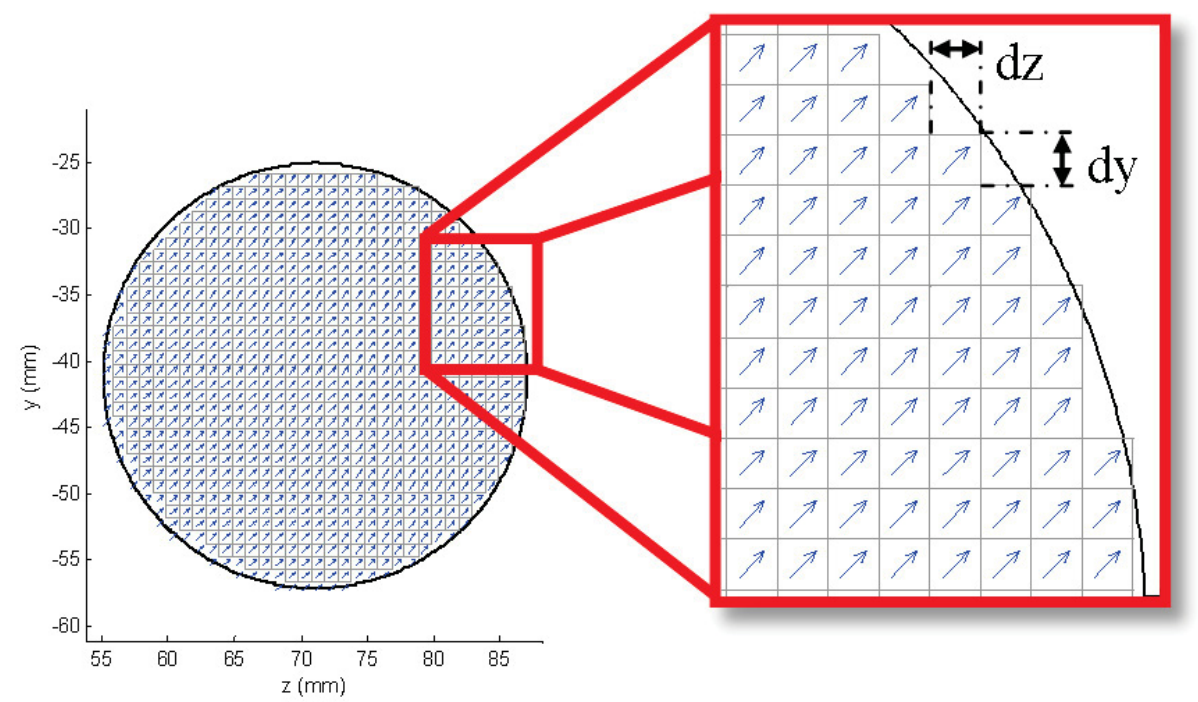

Figure 23. Typical flow area discretization of a coolant channel (Represented vectors are for illustrative purposes and do not accurately represent the flow along the axis of the tube).

The flow rate was calculated by multiplying each vector by $\mathrm{dA}$ and summing over the masked flow field. The following equation described the method to calculate the flow rate $(\mathrm{Q})$.

To determine the accuracy of PIV for the purpose of calculating the bypass ratio, the results were compared to flow rate measurements taken near the inlet of the model using a calibrated turbine flow meter (TFM), accurate to within $\pm 0.05 \%$ of the reading.

Table 7. Flow distribution within the bypass model.

\begin{tabular}{|c|c|c|c|c|c|c|c|c|}
\hline \multirow[b]{2}{*}{$\begin{array}{l}\text { TFM Reading } \\
\text { (L/Min) }\end{array}$} & \multirow[b]{2}{*}{$\begin{array}{c}\text { Stream- wise } \\
\text { Location }(\mathrm{mm})\end{array}$} & \multicolumn{7}{|c|}{ Flow Rate (L/Min) } \\
\hline & & A & $\mathrm{B}$ & $\mathrm{C}$ & $\mathrm{D}$ & E & $\mathrm{F}$ & Gap \\
\hline 351.1 & & 34.8 & 34.4 & 34.7 & 34.0 & 35.7 & 35.5 & 28.8 \\
\hline 579.9 & -1029.5 & 57.6 & 56.5 & 57.1 & 54.0 & 57.2 & 57.2 & 69.5 \\
\hline 1004.1 & & 91.0 & 89.3 & 90.6 & 88.1 & 91.1 & 91.9 & 152.7 \\
\hline 351.15 & & 35.4 & 34.6 & 34.7 & 35.5 & 36.6 & 37.4 & 24.1 \\
\hline 579.9 & -1928.5 & 55.3 & 55.7 & 54.9 & 55.2 & 56.9 & 57.5 & 55.9 \\
\hline 1004.1 & & 90.9 & 90.4 & 91.4 & 91.8 & 94.1 & 96.0 & 142.3 \\
\hline
\end{tabular}

Using the flow rates presented in Table 7, approximate channel Reynolds numbers of 1,750, 2,800, and 4,500 were calculated. Development lengths were predicted for these flows from equations summarized by Munson, Young, and Okiishi (2004). It was estimated that fully developed flow would occur near 3.4, 3.0, and $0.6 \mathrm{~m}$ for the $351.1,579.9$, and $1004.1 \mathrm{~L} / \mathrm{min}$ flow rate cases, respectfully. As the model length is $1.91 \mathrm{~m}$, it was understood that only the highest flow case would be fully developed within the coolant channels. Figure 24 shows the typical variation in time averaged velocity for a flow within a coolant channel. As expected, the lower Reynolds number cases show a developing velocity profile. 


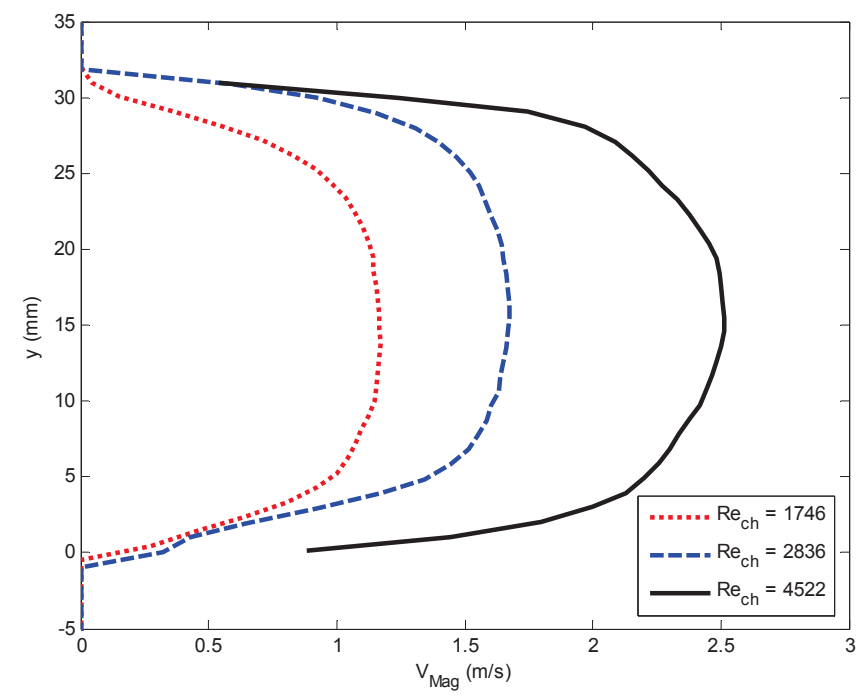

Figure 24. Typical variation of time-averaged velocity in a coolant channel located at $[\mathrm{x}, \mathrm{z}]=[-1029.5$, 103].

Figure 25 illustrates the time averaged velocity through an axial gap located at $[\mathrm{x}, \mathrm{z}]=[-1029.5,0]$. As shown, the flow located at the vertex $(y=0)$ had the highest velocity within the axial gap. Johnson (2011) also observed this flow characteristic in his preliminary calculations, noting that this region was of lower flow resistance. Just below the vertex, the velocity gradually increased toward the perimeter of the model. Recalling the gap was not uniform, and the width of the gap increased from 5.47 to $6.2 \mathrm{~mm}$ between the vertex and the perimeter of the model; it was understood that higher velocities occurred where the gap was largest.

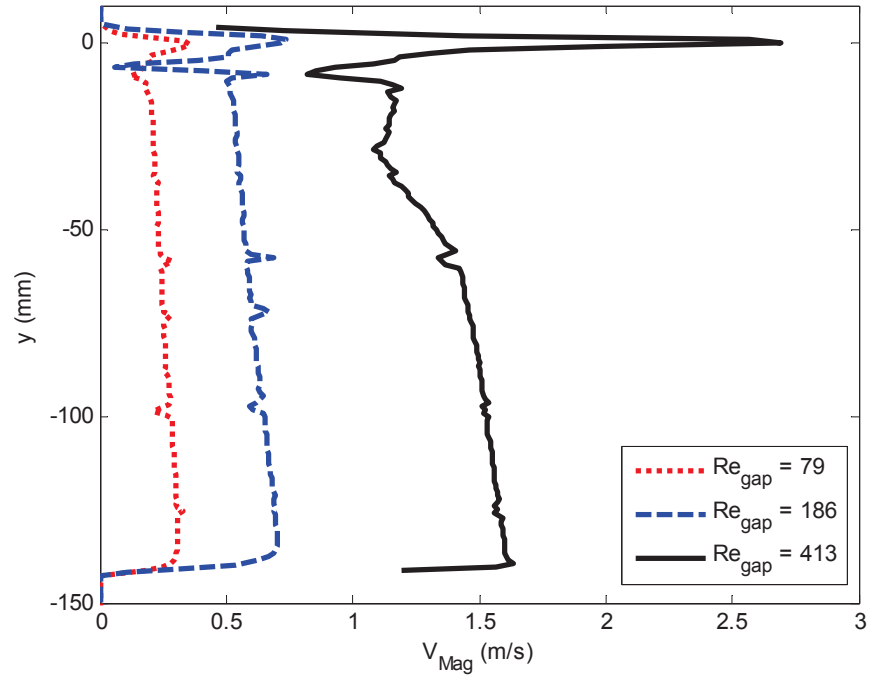

Figure 25. Variation of time-averaged velocity in a gap located at $[\mathrm{x}, \mathrm{z}]=[-1029.5,0]$.

Flow rate calculations were computed for each case in the test matrix. It was observed that calculated flow rate error varied between 6.2 and $10.5 \%$ when compared to the TFM reading. For the lowest and highest flow rates, the bypass flow ratio ranged from 7.3 to $16.94 \%$. Because of lack of optical access in the upper three tubes of the model, it was assumed these velocity quantities were inaccurate. For this reason, the average velocity of all six lower coolant channels was superimposed on the upper channels to estimate a more realistic flow rate. Figure 26 illustrates the superposition principle using data from Figure 22. In this case, the average flow of channels A, B, C, D, E, and F was superimposed on the upper three channels $\mathrm{G}, \mathrm{H}$, and I. 


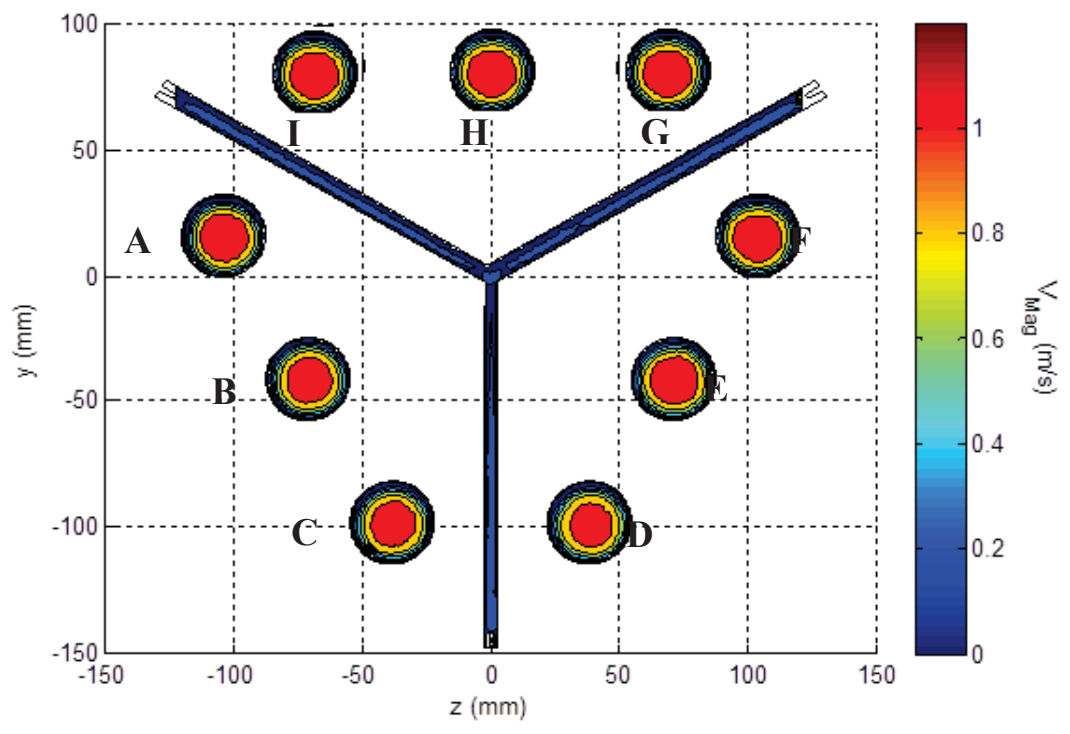

Figure 26. Superimposed velocity contour plot (superimposed data on upper three tubes).

Table 8 gives flow rate calculations, errors, and bypass flow ratios, considering the superimposed data. As expected, the calculated flow rate error decreased substantially. However, manipulating the data had little effect on the bypass flow ratio.

Table 8. Bypass flow results for a scaled 3-mm gap width (superimposed data on upper three tubes).

\begin{tabular}{|ccccccc|}
\hline $\begin{array}{c}\text { TFM } \\
\text { Reading } \\
\text { (L/Min) }\end{array}$ & $\begin{array}{c}\text { Stream-wise } \\
\text { Location } \\
(\mathrm{mm})\end{array}$ & $\begin{array}{c}\text { PIV } \\
\text { Calculated } \\
\text { Flow Rate } \\
\text { (L/Min) }\end{array}$ & $\begin{array}{c}\text { Calculated } \\
\text { Flow Rate } \\
\text { Error }(\%)\end{array}$ & ReCh & ReGap & $\begin{array}{c}\text { Bypass Flow } \\
(\%)\end{array}$ \\
\hline 351.15 & & 342.50 & 2.46 & 1,746 & 79 & 8.40 \\
579.9 & -1029.5 & 579.01 & 0.015 & 2,836 & 186 & 12.00 \\
1004.1 & & 965.55 & 3.84 & 4,522 & 413 & 15.82 \\
351.15 & & 345.46 & 1.62 & 1,789 & 66 & 6.96 \\
579.9 & -1928.5 & 558.94 & 3.61 & 2,778 & 150 & 9.99 \\
1004.1 & & 974.14 & 2.98 & 4,618 & 382 & 14.61 \\
\hline
\end{tabular}

The bypass flow in the upper block was observed to be higher than that of the lower block for all flow rates within the test matrix. Accordingly, it was understood that the flow redistributes via the radial gap, entraining oil into the coolant channels and starving the gap in the lower block.

In relating the present study to the CFD calculations, it was noted that the flow rates considered by Johnson (2011) were considerably higher than those achieved in the present study. For this reason, only the high flow experimental case was effectively compared $\left(\mathrm{Re}_{\mathrm{Ch}} \approx 4,500\right)$. A graph of the experimental and CFD bypass flow ratio results versus channel Reynolds numbers is shown in Figure 27. For the flow rate of $1231.8 \mathrm{~L} / \mathrm{min}$, CFD estimated the channel and gap Reynolds numbers as 5,440 and 524. As expected, the present study showed that the lower flow rate of $1004 \mathrm{~L} / \mathrm{min}$ produced Reynolds numbers of 4512 and 412 for the coolant channel and gap, respectively. However, in examining the bypass ratio for both cases, it appears that the CFD under predicts the amount of flow traveling through the gaps in both the upper and lower blocks. 


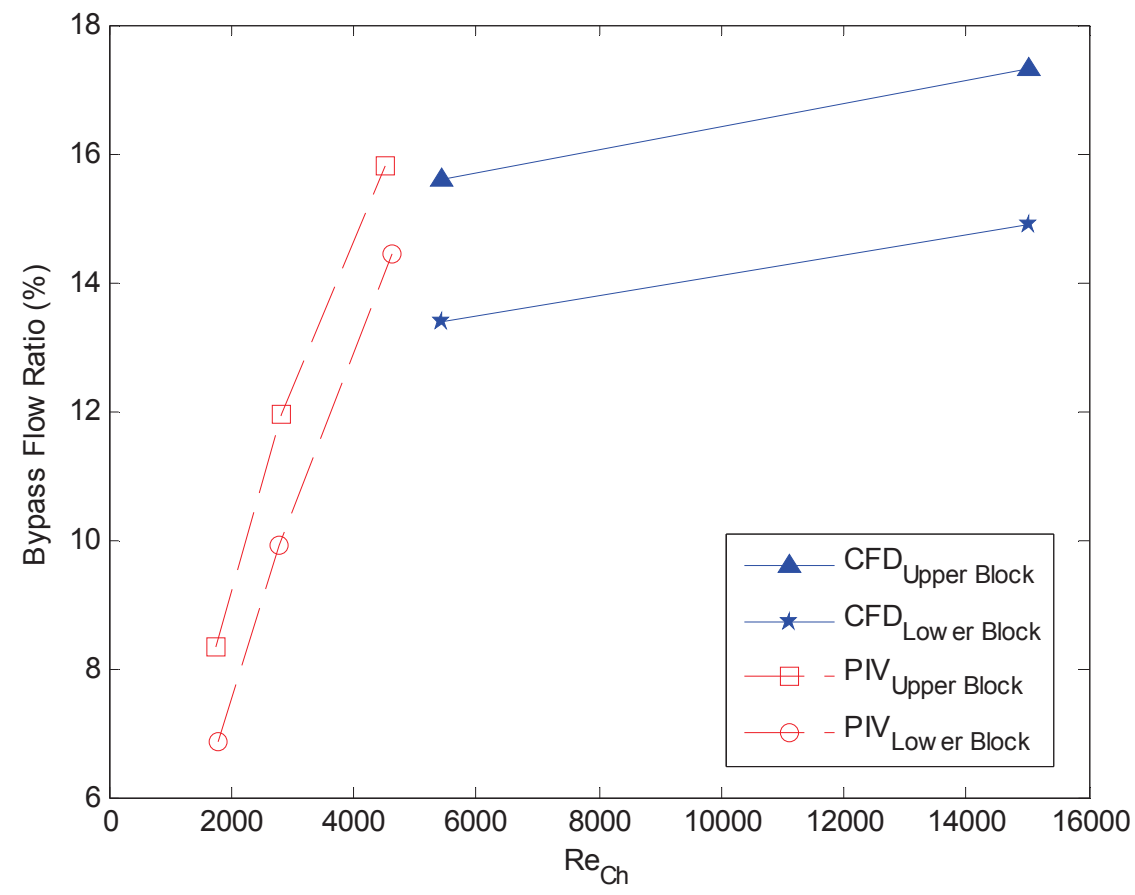

Figure 27. Comparison of CFD and experimental data.

\subsubsection{Summary}

Velocity measurements were taken in the MIR facility to measure the anticipated bypass flow associated with a prismatic GT-MHR. A model that represented a stacked junction of six partial fuel blocks with nine coolant tubes and axial and radial gaps was manufactured and installed in the MIR. SPIV was then employed to measure the flow field within. Measurements were taken in three locations along the length of the model: in the upper plenum, in the midsection of the large fuel blocks, and in the midsection of the small fuel blocks. Flow rates were calculated for the coolant channels and gaps for comparison. The bypass ratio was estimated to range from 6.8 to $15.8 \%$ for the considered flow rates. When compared to pretest calculations, the CFD analysis appeared to under predict the flow through the gap.

\subsection{Bypass Transitional Flow Measurements of a Flat Plate}

Bypass transitional flow is a specific flow regime that falls between the laminar and turbulent flow regime of wall bounded flows that has "bypassed" the Tollmien-Schlichting waves by the means of freestream turbulent eddies. It should be noted that the use of the term "bypass" has changed definition in this section from the previous section. Where the previous section used the term bypass as a means of describing the flow within the interstitial gaps of the prismatic core blocks, the use of bypass in this section is referring to bypassing natural waves that form when wall-bounded flow is transitioning from laminar to turbulent.

\subsubsection{Flat Plate Design}

The design process was analyzed from many aspects of wall-bounded flow behavior. A restart of a flat plate experiment already performed at the MIR was beneficial to the current study as the parts and materials were reused. The need for 'bypass' transitional flow was the most important design parameter as this was the crux of the study. Bypass was achieved by both increasing the freestream turbulence intensity and incorporating an adverse pressure gradient (APG) on the normal component of the flow. The turbulence eddies in the freestream were introduced into the flow by the means of a turbulence grid upstream of the flat plate experiment, which consisted of 21 horizontal round bar stock that has a pitch of 1 inch, as seen in Figure 28. 


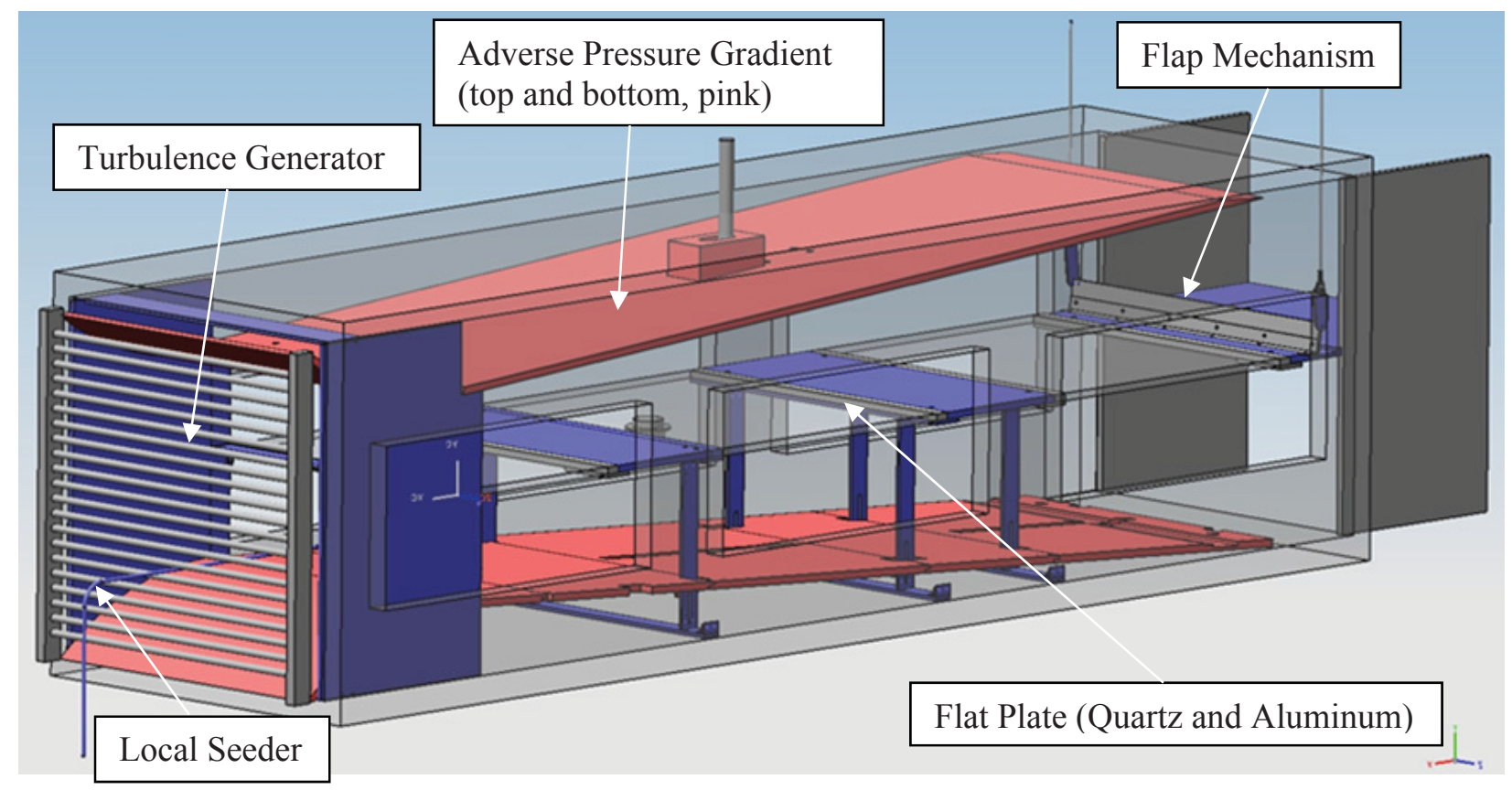

Figure 28. Isometric view of adverse pressure gradient flat plate study with turbulence generator.

The APG was utilized by first incorporating into the design a "bell-mouthed" shaped curve, located prior to the leading edge of the plate. To achieve symmetrical flow, the APG needed to be incorporated on both the top and bottom of the flat plate. This allowed for the fluid to be uniformly restricted as it flowed into the bell-mouth contraction, then to expand away from the plate just over and below the leading edge. The APG was designed with an angle of 3.5 degrees from the horizontal.

The plate was positioned slightly above the horizontal centerline of the tunnel, as flow was slightly restricted by the support structures holding the plate in position. This compensated for cross-sectional area being too large on the top (data acquisition) side of the plate. Furthermore, a flap mechanism was installed at the furthest downstream location on the plate. It was found that the flap mechanism actually provided very little assistance in balancing the flow and was left in the down position.

Another design specification was injecting seeding directly into the boundary layer at the leading edge. It was presented by Kahler et. al. (2006) that a local seeder could be placed just next to the leading edge without disturbing the flow. A 1/4-in. tube was used as a local seeder and placed directly next to the leading edge (as seen in Figure 29). This was in line with a low-flow gear pump that was set at a minimal flow rate as to not disrupt the bulk flow near the leading edge when the seeded fluid exited the nozzle. The gear pump drew fluid from a tank that was densely seeded (in comparison to the bulk of the fluid in the actual oil tunnel) and heated to matching temperature using the MIR system's secondary heating loop. 


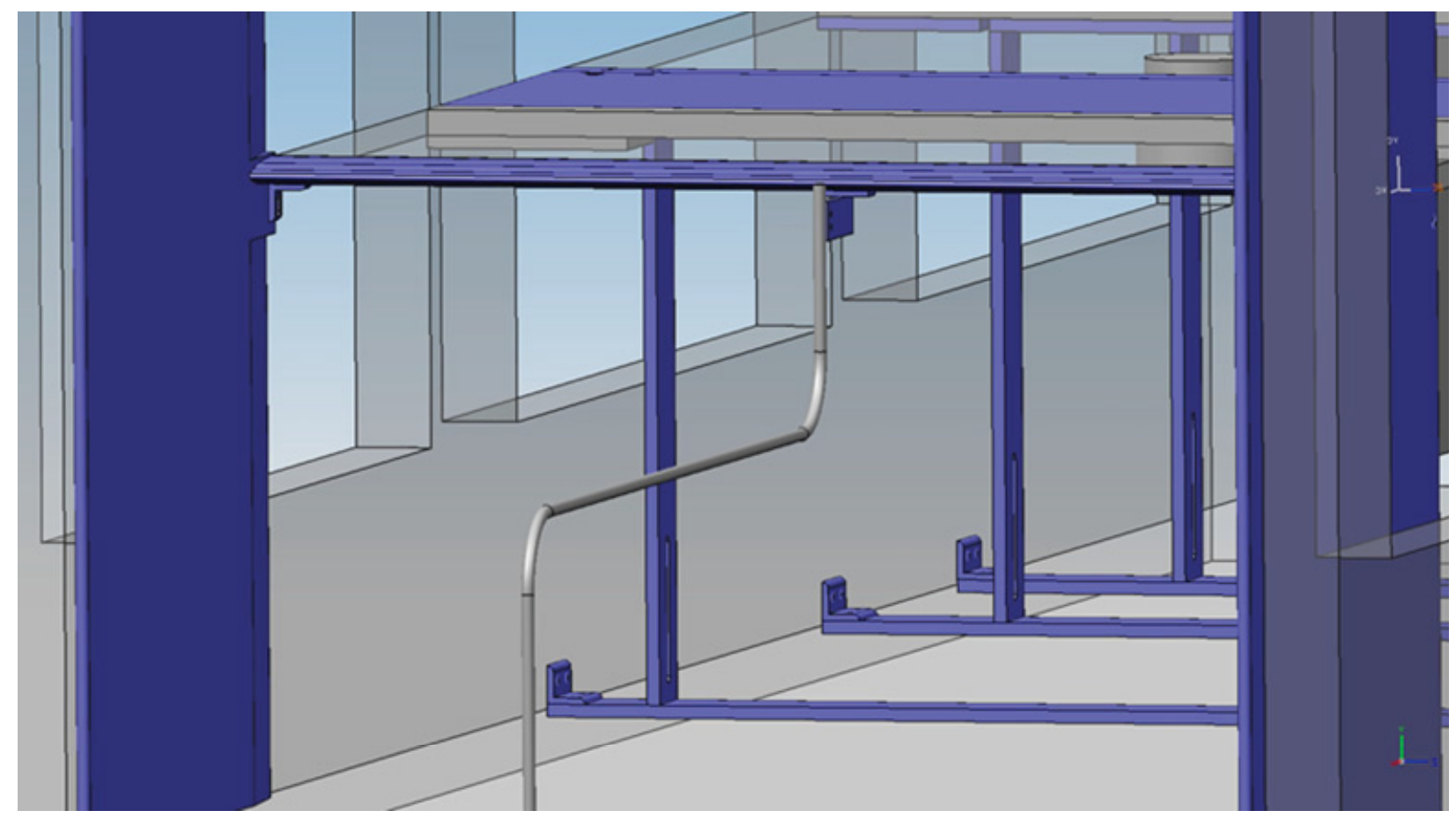

Figure 29. Detail of the local seeding tube fastened near the leading edge of the flat plate.

\subsubsection{Benchmark Studies}

To calibrate the MIR system and flat plate study, two simplistic studies were performed in the oil tunnel to benchmark against readily available flat plate data: (1) a zero pressure gradient (ZPG) without the turbulence generator (TG) installed (as shown in Figure 30), and (2) ZPG with the TG installed. The ZPG case was achieved by assuming the tunnel walls were a "negligible" favorable pressure gradient. The former was found to be in direct comparison to the Blasius profile, holding that the case was in fact laminar down the length of the plate. The later benchmark of ZPG with a TG was mainly used as a connection between the laminar flow found in the ZPG without a TG and the fully installed APG with a TG. Both the ZPG cases (with and without a TG) were helpful in determining transitional behavior in the overall experiment. PIV data very near walls break down and overestimate the flow in this region, due mainly to the discrete size of the IWs always measuring some flow within. 


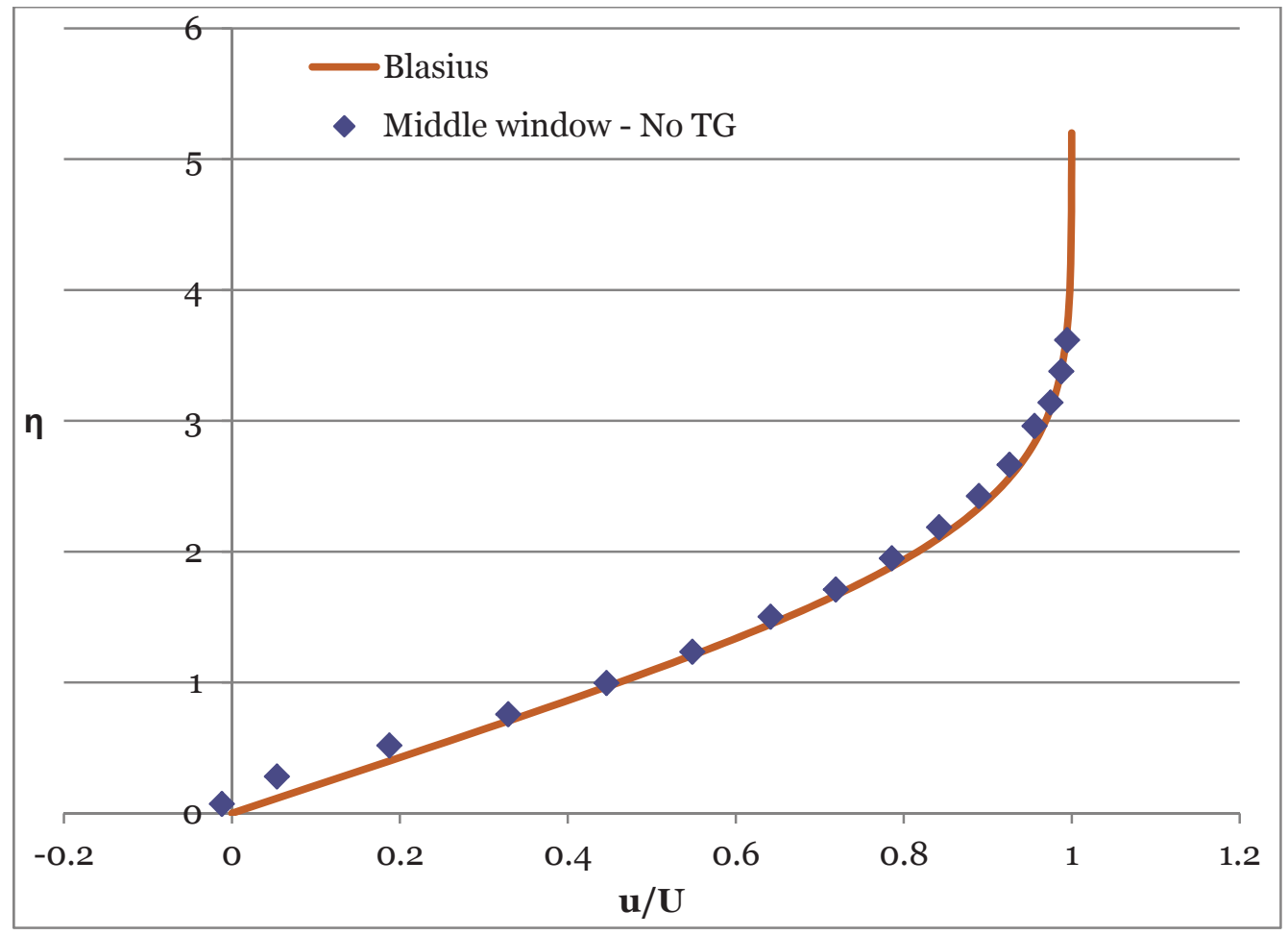

Figure 30. Experimental data achieved in the center window of the MIR during the ZPG without a TG installed.

\subsubsection{Test Plan}

Planar PIV was used to capture the flow over the flat plate. Two FOVs were used: large scale FOV (at approximately 6 in.) and a mezzo FOV (at approximately 0.5 in.). The image plane was located at the centerline of the tunnel/plate with the normal axis in the tunnel/plate span wise direction. The camera was set up perpendicular to the image plane just above the flat plate, with the bottom of the image slightly viewing the top surface of the flat plate. As much data as could be acquired down the length of the plate within the three windows of the tunnel was recorded, with the camera traversing downstream from the leading edge of the plate. The tunnel's main axial pump was ramped up to maximum capacity allowed by the MIR specification sheet.

4,000 image pairs were recorded at each location in each FOV (both large and mezzo). This was determined from the Uzol study mentioned above as the measurements from the image sets appeared to converge around 4,000 image pairs. Also, to further improve the wall normal gradient measurements, the time between image pairs in the mezzo FOV was varied based on local flow conditions to utilize two different time stamps. One was curtailed as a "long" dt while the other was a "short" $d t$; in this sense, the longer dt could resolve the slower flow near the wall (even down to the zero velocity at the wall), and the short dt better resolved the fluid flow that bridges the large FOV data sets to the mezzo FOV data sets, as shown in Figure 31. In contrast, only every other location was recorded, as the wall normal gradient was found to be more crucial in accurate measurement than to have a streamwise continuous measurement. This allowed for saving space on the hard drives for the important measurements. 


\section{Large FOV data acquisition}

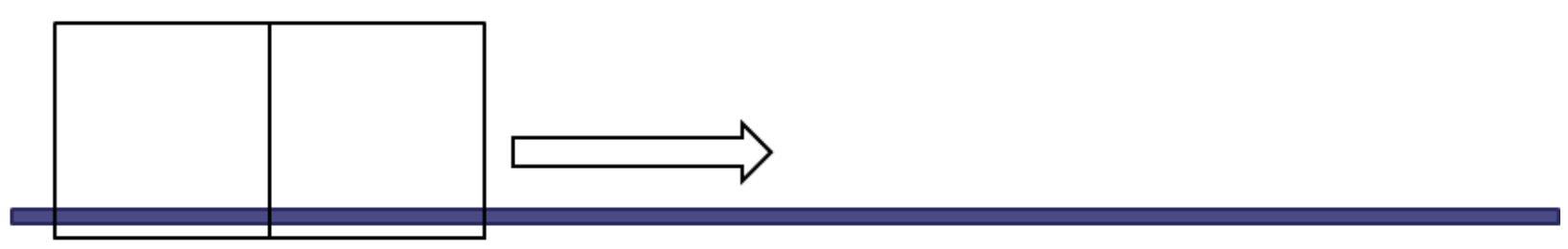

\section{Flat plate}

\section{Mezzo FOV data acquisition}

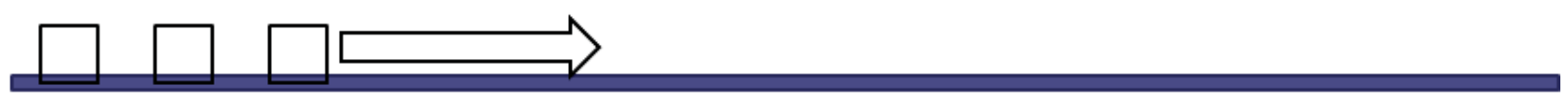

\section{Flat plate}

Figure 31. APG case having large FOV and mezzo FOV (by varying the dt between camera images).

\subsubsection{Flow Rate Analysis}

The quantity of data obtained from this study is staggering. Just beyond 10 terabytes of data were recorded at the MIR facility, and analyzing its entirety is currently underway. Some preliminary results have been found to show reasonable behavior of bypass transition within the confines of the oil tunnel.

The freestream conditions of the ZPG with the TG (see Figure 32) show the initial freestream turbulence intensity as a percent of the fluctuations to the freestream streamwise velocity component to start out at $\sim 9 \%$ near the leading edge of the plate and decrease exponentially to $\sim 4.5 \%$. Ideally, the freestream flow is uniform, but due to the tunneling effects of the MIR test section, there is a slight increase in freestream velocity. The Reynolds number, based on x position and boundary layer thickness, grows along the length of the plate.

To further understand the flow phenomenon, and making sure to aquire transistional flow regime within the tunnel, experimental results were compared to a Direct Numerical Simulation (DNS) data set. Figure 33

shows both the experimental ZPG and the DNS ZPG as skin friction coefficient (Cf) versus plate location. It is the main focus of this study to observe the minimum value of $\mathrm{Cf}$, as it immediately follows the transitional region of the flow. Also, on this graph is a plot of the experimental APG and its DNS comparison, APG4 (where the 4 in this label represents the amount of lamda $=0.04$, lamda being the dimensionless pressure gradient parameter $\lambda=\delta / \tau_{\mathrm{w}}{ }^{*} \mathrm{dp} / \mathrm{dx}$ ). The third window of data in this set is still under investigation, but the minimum in Cf can be observed slightly downstream from that of the APG4 Cf minimum. This is consistent, as the experimental APG has a slightly lower pressure gradient than what was modeled in the DNS. 

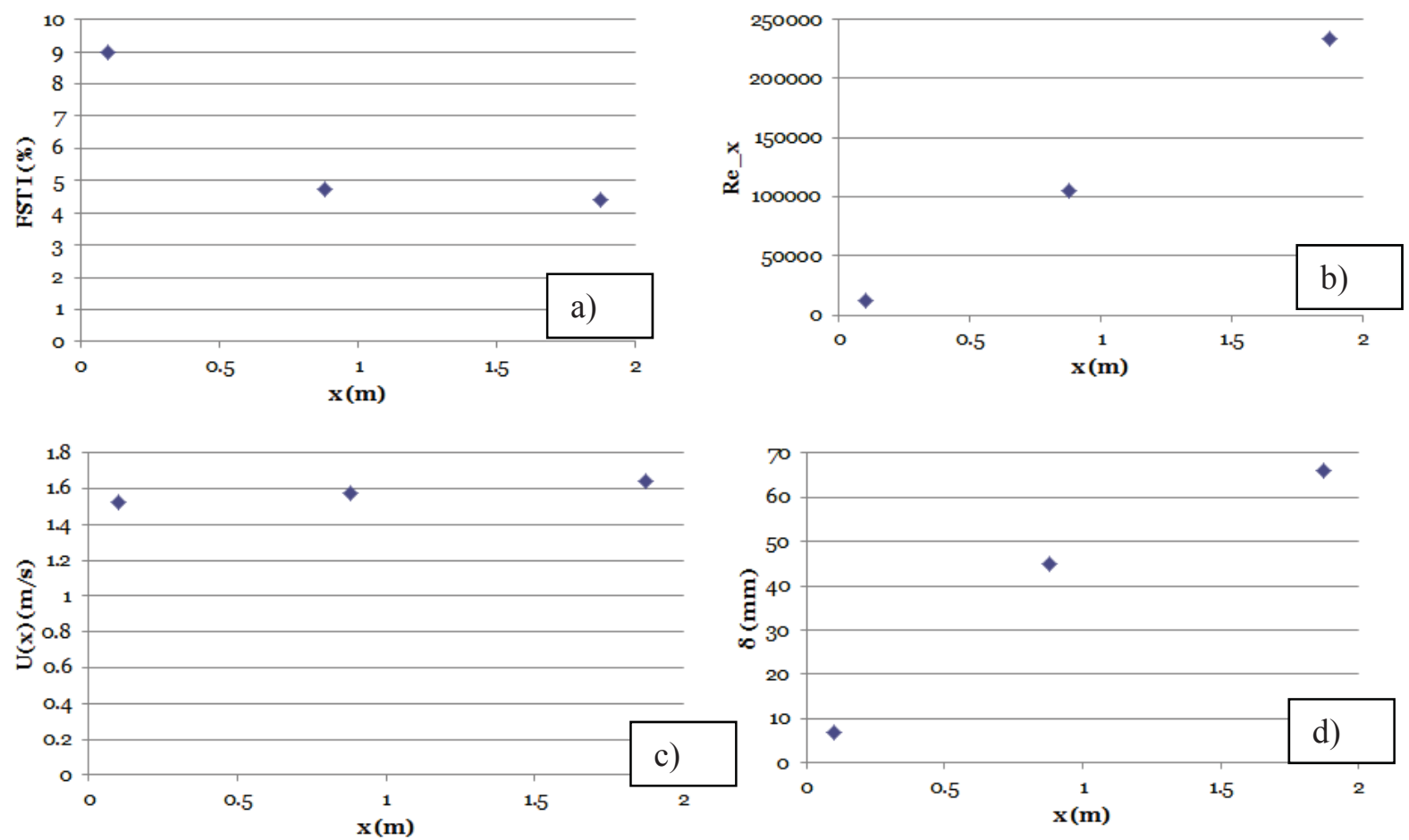

Figure 32. ZPG with TG installed preliminary results: (a) the freestream turbulence intensity versus plate location, (b) Reynolds number based on x versus plate location, (c) freestream streamwise velocity component versus plate location, and (d) boundary layer thickness versus plate location.

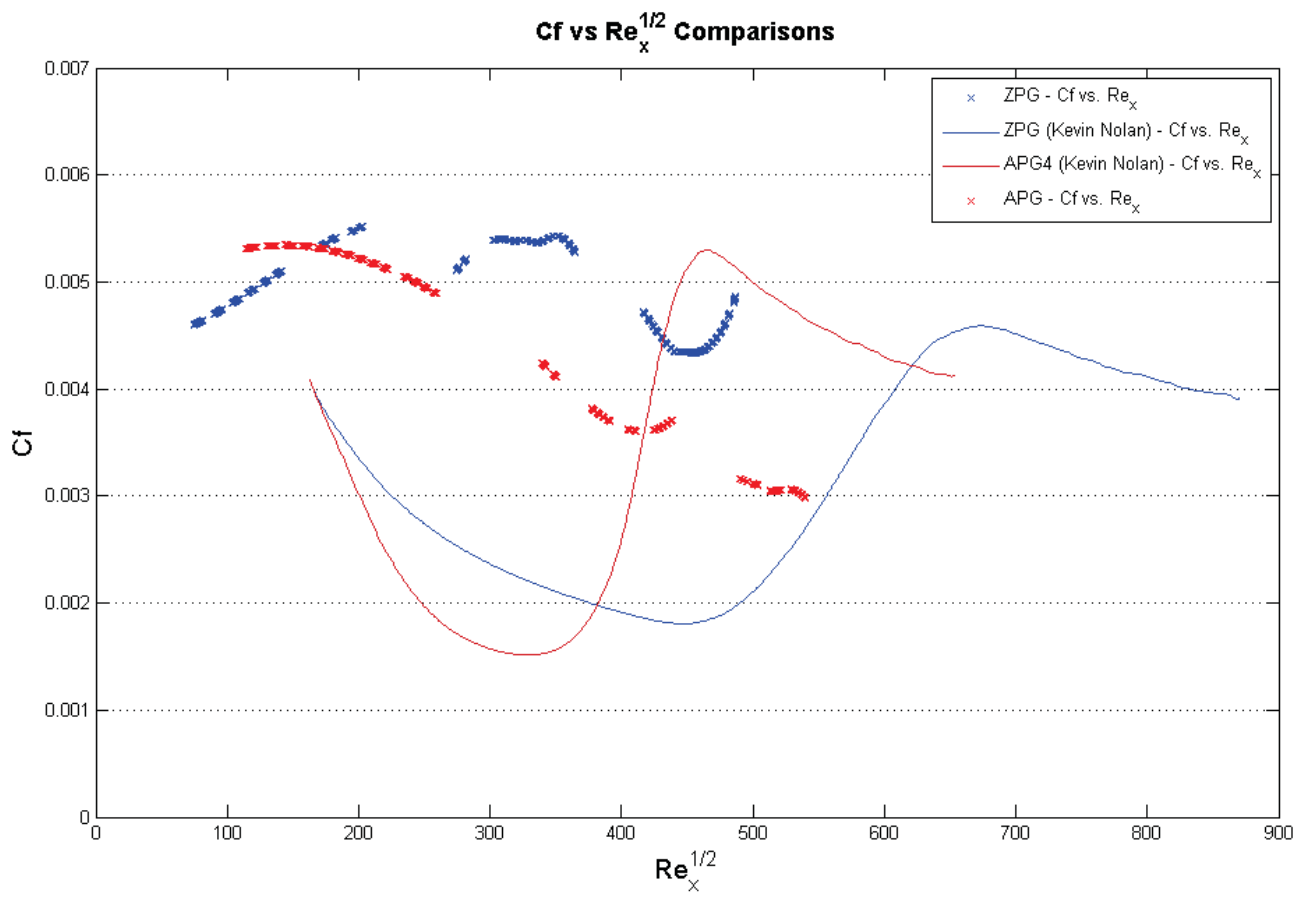

Figure 33. Comparison of direct numerical simulation with experimental results from the MIR flat plate experiment. 


\subsubsection{Conclusions}

A flat plate was installed into the MIR oil tunnel to quantify entropy generation within the bypass transitional region of wall-bounded flow. The study is still under investigation, but preliminary results have shown that transitional flow has been achieved within the confines of the tunnel test section. This was accomplished mainly by the use of a TG and an APG (diverging plate) that feeds the boundary layer and brings the transition to turbulence closer to the leading edge. 


\section{DEVELOPING AN UNCERTAINTY QUANTIFICATION METHOD FOR THE MIR FACILITY}

The overall objective of developing an uncertainty quantification method for the MIR facility is to characterize the accuracy of flow data measured in the facility using PIV. The MIR is commonly used for CFD validation studies, which require rigorous uncertainty quantification. Until this work, such uncertainty quantification was not possible. These results are applicable to the MIR measurements as well as the broader PIV community. INL showed early leadership in the field of PIV uncertainty by funding the first PIV Uncertainty Workshop, which was organized by the principal investigators. The Laboratory Directed Research and Development work described here capitalizes on that start and places INL at the forefront of PIV uncertainty work.

The uncertainty is affected by the numerous parameters present in the instrumentation chain, and in the vast majority of the cases, those uncertainties are interconnected and coupled; however, one of the most critical is the uncertainty associated with the PIV algorithm itself. This is arguably the most challenging and complex part of the process. Within the scope of this project, two-research teams (USU and VT) are collaborating with INL to deliver two complementary and integrated approaches that quantify PIV uncertainty and provide a ready-to-use software package that is able to serve INL needs. It is important to note that, to date, there is no accepted methodology for quantifying the uncertainty associated with individual vector evaluations. Both the methodologies developed are discussed here, and further details are provided in Appendix B.

\subsection{Uncertainty Surface Method}

The USU deliverable is a code used to estimate the uncertainty of a PIV measurement based on the Uncertainty Surface Method, which was developed under a previous INL Laboratory Directed Research and Development program. This method uses synthetic test data to determine a PIV code's error response to various inputs. While, in principle, any number of inputs can be tested, thus far, four have been used: flow shear, particle displacement, particle image density, and particle image size. The results of these tests are stored in a multidimensional "Uncertainty Surface," which the uncertainty code uses in conjunction with measurements of each of the inputs to determine the uncertainty for each measurement.

The Uncertainty Surface Method determines the relationship between various error sources and estimates their contribution to PIV uncertainty. In their study, Timmins et al. (2012) considered particle image diameter, particle density, particle displacement, and velocity gradient as possible sources for PIV uncertainty. It is important to note that these sources were chosen arbitrarily. They were used to create an "uncertainty surface" (or baseline) specific to a PIV algorithm and known flow field. These parameters were varied to create an array of synthetic images that mimic known flows and were then evaluated against the true flow to establish uncertainty. The code estimated the velocity uncertainty for each vector. This method can be adapted to any PIV system, provided that the relevant error sources can be identified for a given experiment and the appropriate parameters can be quantified from the images.

The code was based on the following procedure, as described in detail in Appendix B and Timmins et al. (2012):

1. Identify and select contributors to PIV error (particle image size, seeding density, shear rate, etc.)

2. Generate synthetic images for rectilinear flow, varying each contributor identified in Step 1

3. Compute vector fields for the synthetic images and compare them to known solutions to find the errors as a function of each of the parameters

4. Compute the uncertainty estimates from the distribution of errors found in Step 3 and from the uncertainty surface

5. Estimate the parameters found in Step 1 
6. Combine the estimates from Step 5 with the uncertainty surface from Step 4 to determine the uncertainty for each vector

7. Verify the methods' effectiveness by generating new synthetic images for several flows and comparing the true solution of known flows to the calculated.

The reliability of this methodology was validated using known flow fields, so the actual error could be determined. The analysis showed that, for most flows, the uncertainty distribution obtained using this method fit the confidence interval.

Currently, this technique shows promise to quantify the uncertainty that stems from Fast Fourier Transform-based, cross-correlation PIV algorithms, but it is left to the discretion of the researcher to determine which parameters are most critical to include in the uncertainty analysis. The values of other uncertainties associated with PIV, which cannot be incorporated into this method, can be root-sum-squared with the uncertainty surface results to obtain a total combined uncertainty value. Also, it is important to note that this method makes no attempt to account for particle motion normal to the laser plane; however, ongoing work suggests this technique is capable of being applied to three-component PIV studies.

At the start of this project, USU's code, based on VT's PRANA PIV code, could compute PIV vectors as well as the uncertainty of a PIV measurement because of particle displacement, particle image density, particle image diameter, and shear. The uncertainty code was integrated with the PIV code, and both the vectors and uncertainty were computed in one processing step. Since both the vector computation and the uncertainty estimate require access to the raw image data, this was deemed an efficient method. However, this code was inflexible in terms of the PIV code to be used, and no means existed to use the uncertainty method with commercial PIV software.

Since INL uses commercial PIV software (called DaVis), the first USU task was to separate these two functions. Currently, the Uncertainty Surface Code stands alone and can use input from any PIV code for which a surface could be generated.

The methods for estimation of particle image diameter and density used at the start of this project were found to not be robust when applied to real data. Methods for the estimation of particle image diameter were found in the literature and incorporated into the uncertainty surface code. Methods for estimation of particle image density were developed at USU during the last months, and further details can be obtained from Appendix B.

\subsection{Cross-correlation Peak Ratio Method}

The VT team has been pursuing an elegant method based on the hypothesis that the cross-correlation contains information about uncertainty. The results to date show that the ratio of the largest correlation peak to the second largest is an excellent predictor of uncertainty for the Robust Phase Correlation (RPC) displacement estimator implemented in PRANA. The peak ratio is also a good predictor of the uncertainty for the SCC displacement estimator, which is more commonly used. The details of this study are provided in Appendix B.

The VT team pursued a method based on the peak ratios produced after applying the PIV cross-correlation algorithm to each IW. The peak ratio is defined as the ratio between the highest to second highest peak in each correlation plane. Using the RPC algorithm in PRANA (a PIV image processing software produced by VT), Charonko et al. (2013) showed that the peak ratio was an acceptable predictor of the algorithm's uncertainty regardless of flow condition or image quality. They used an analytical model, derived from synthetic data sets to compute the uncertainty bounds at a 95\% confidence interval for several artificial and experimental flow fields. They found that the resulting errors closely matched the predicted uncertainties.

For comparison, the peak ratio method was also applied to the SCC algorithm, which is more commonly used in other PIV software. It was determined that the SCC peak ratio results produced a menial uncertainty estimate. 
Using the peak ratio (Q), Charonko et al. (2013) modeled the uncertainty for an SCC-based measurement as:

$U n_{S C C}=\left[\left(13.1 e^{\left(-\frac{1}{2}\left(\frac{Q-1}{0.317}\right)^{2}\right)}\right)^{2}+\left(0.226 \frac{1}{Q}\right)^{2}+(0.08)^{2}\right]^{1 / 2}$ using:

Similar relationships were also given for the RPC-based approach, and better curve-fits were obtained

$U n_{R P C}=\left[\left(9.76 e^{\left(-\frac{1}{2}\left(\frac{Q-1}{1.139}\right)^{2}\right)}\right)^{2}+\left(1.41 \frac{1}{Q}\right)^{2}+\left(0.1 .7(10)^{-5}\right)^{2}\right]^{1 / 2}$.

For a more in-depth review of the methodologies, the reader is referred to Appendix B of this report.

\subsection{Path Forward}

A PIV "wiki" has been built on the following site (www.piv-online.org) with input from the VT and USU team. Thus far, this wiki concentrates on best practices, although it is built to become much broader, as shown in Figure 32. Shortly, the team will invite several international PIV experts to contribute to the wiki. Once this process has had sufficient time, a broader announcement will be made.

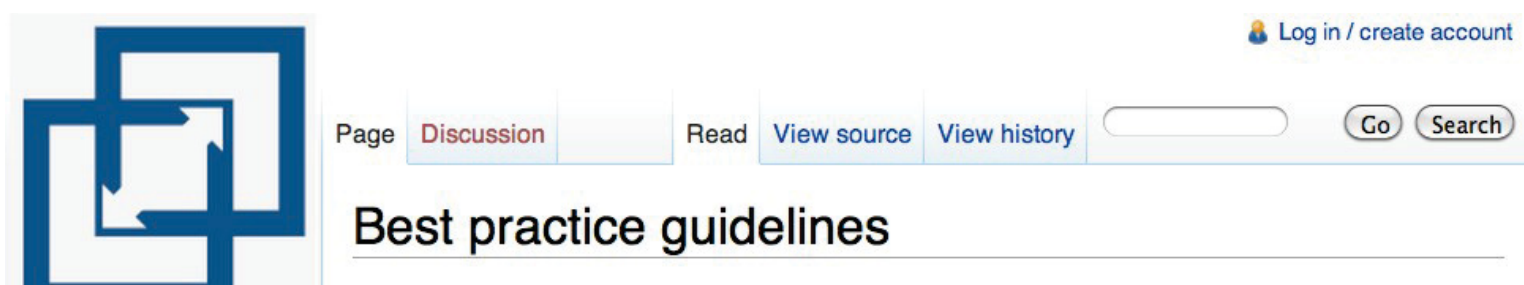

You can also visit the Stereo PIV Best Practices Page

Navigation

Contents [hide]

Main page

Community portal

Current events

Recent changes

Random page

Help

Toolbox

What links here

Related changes

Special pages

Printable version

Permanent link

Contents [hide]
1 How to plan and execute a good planar PIV experiment and a zero ${ }^{\text {th }}$ order
shakedown
2 Experimental design
3 Particles and illumination
4 Images and recording
5 Velocity estimation
6 Outputs

\section{How to plan and execute a good planar PIV experiment and a zero ${ }^{\text {th }}$ order shakedown}

The following list provide general suggested guidelines and suggested steps in the process of a typical planar two-component PIV experiment. This list is not intended to be either all inclusive or correspond to a strict process. All experiments are different and as such appropriate changes and adjustments are always needed.

\section{Experimental design}

1. Determine the relevant velocity, length and time scales. Calculate all relevant dimensionless numbers for the flow.

Figure 34. Screen shot from PIV-Online wiki.

The remaining task for this year is the cross testing of the methods. This effort is currently ongoing and involves testing and comparing each method against known flows using real experimental data, including that obtained from the Bypass Flow Experiment. As this data is three-component, the INL's interest in PIV uncertainty extends to SPIV measurements and is scheduled to be addressed in the upcoming year. It is important to note that SPIV works by computing a two-component velocity field from each camera and then using calibration images to map locations in one camera's view to the other camera's view. The uncertainty 
of an SPIV measurement can thus be assessed by assessing the uncertainty of each of the two-component fields, decomposing these results into a single three-component uncertainty field, and then adding estimates of biases from the stereo calibration process.

\subsection{Summary}

The main objective of this study was to examine current uncertainty quantification methodologies for the MIR flow facility, which is needed for data quality assessment and for CFD code validation. Despite extensive research on the accuracy of various DPIV implementations, to date there is no accepted methodology for quantifying the uncertainty associated with individual vector evaluations. The current effort helps develop a framework for PIV uncertainty that focuses on the PIV algorithm, which arguably is one of the most challenging and complex parameters of the total uncertainty, and must be quantified with other important factors. Two methodologies were introduced to establish uncertainty for PIV measurements in the MIR facility; specifically, the Uncertainty Surface and Cross-correlation Peak Ratio Methods that are currently being developed by USU and VT. These uncertainty quantification methodologies can potentially lead to higher quality MIR data with accurate and reliable uncertainty quantification. They can be a tremendous asset to the ongoing efforts to validate advanced computational methods. 


\section{REFERENCES}

Adrian, R. J. and J. Westerweel, 2010, Particle Image Velocimetry, Cambridge University Press, p. 558.

Adrian, R. J., 1991, "Particle-Imaging Techniques for Experimental Fluid-Mechanics," Annu. Rev. Fluid Mech., Vol. 23, pp. 261-304.

Adrian, R. J., 1997, "Dynamic ranges of velocity and spatial resolution of particle image velocimetry." Measurement Science \& Technology, Vol. 8, pp. 1393-1398.

Adrian, R. J., 2005, Twenty Years of Particle Image Velocimetry, Experiments in Fluids, Vol. 39, pp. 159169.

Adrian, R. J., and Westerweel, J., 2011, Particle Image Velocimetry, Cambridge University Press.

Astarita, T., 2007, “Analysis of weighting windows for image deformation methods in PIV," Experiments in Fluids, Vol. 43 No. 6, pp. 859-872.

Brady, M. R., S. G. Raben, and P. P. Vlachos, 2009, "Methods for Digital Particle Image Sizing (DPIS): Comparisons and improvements," Flow Measurement and Instrumentation, Vol. 20, No.6, pp. 207-219.

Charonko, J., Vlachos, P., 2013, "Estimation of Uncertainty Bounds for Individual PIV measurements from Cross-correlation Peak Ratio," Submitted to Meas. Sci. Tech. on October 21, 2012.

Conder, T.E., Skifton, R.S., Budwig, R.S., 2012, "Particle Image Velocimetry Measurements in a Representative Gas-cooled Prismatic Reactor Core Model: Flow in the Coolant Channels and Interstitial Gaps," Proceedings of the ASME International Mechanical Engineering Congress and Exposition, Houston, TX.

Fore, L. and A. Tung, 2005, "Nonlinear Temporal Filtering of Time-Resolved Digital Particle Image Velocimetry Data," Exp Fluids, Vol. 39, pp. 22-31.

General Atomics, 1996, "Gas Turbine-Modular Helium Reactor (GT-MHR) Conceptual Design Description Report," Doc. 910, 720 Rev1.

Guezennec, Y. G. and Kiritsis, N., 1990, "Statistical Investigation of Errors in Particle Image Velocimetery,” Exp. Fluids, Vol. 10, pp. 138-146.

Gui, L. and W. Merzkirch, 2000, "A comparative study of the MQD method and several correlation-based PIV evaluation algorithms," Experiments in Fluids, Vol. 28 No. 1, pp. 36-44.

Hain, R. and C. Kähler, 2007, "Fundamentals of multiframe particle image velocimetry (PIV)" Experiments in Fluids, Vol. 42 No. 4, pp. 575-587.

Huang, H., Dabiri, D., and Gharib, M, 1997, “On Errors of Digital Particle Image Velocimetery,” Meas. Sc. Technol., Vol. 8, pp. 1427-1440.

Huang, H., D. Dabiri and M. Gharib (1997). “On errors of digital particle image velocimetry." Measurement Science and Technology 8(12): 1427.

INEEL, 2005, "Next Generation Nuclear Plant Research and Development Program Plan," INEEL/EXT-05-02581.

INL, 2010, “Next Generation Nuclear Plant Methods Technical Program Plan,” INL/EXT-06-11804.

Specialist Committee on Uncertainty Analysis of $25^{\text {th }}$ ITTC, 2008, "Uncertainty Analysis Particle Imaging Velocimetry," International Towing Tank Conference, ITTC-Recommended Procedures and Guidelines, September 2008, CONF-25, Vol. 7.5.01-03-03, Rev. 00, pp. 1-12.

Johnson, R., 2011, "Pre-Test CFD Calculations for a Bypass Flow Standard Problem," ASME IMECE, Denver, CO.

Kaburaki, H., Takizuka, T., 1987, "Effect of Crossflow on Flow Distribution in HTGR Core Column," J. of Nuclear Sci. and Tech, 24, pp. 516-525. 
Kahler, C. J., 2006, "Wall-shear-stress and near-wall turbulence measurements up to single pixel resolution by means of long-distance micro-PIV," Experiments in Fluids, DOI 10.1007/s00348-006-0167-0.

Keane, R.D., Adrian., R.J., 1992, "Theory of Cross-correlation Analysis of PIV Images," Applied Scientific Research, Vol. 49, pp.191-215.

Kompenhans, J., et al., 2007, "Particle image velocimetry," a practical guide with 42 tables, p. 448.

LaVision, 2002, DaVis PIV Seminar, 19-21 October, 20010, Ypsilanti, MI.

Marxen, M., et al., 2000, "Comparison of Gaussian particle center estimators and the achievable measurement density for particle tracking velocimetry." Experiments in Fluids, Vol. 29 No. 2, pp. $145-153$.

McEligot, D. M., Budwig, R. S., Crepeau, J., 2010, Fundamental Fluid Physics Studies for Energy Efficiency and Sustainability, DOE EPSCoR No. \#DE-SC0004751.

McEligot, D. M., Mcllroy, H. M., and Johnson, R. C., 2007, Estimated Uncertainties in the Idaho National Laboratory Matched-Index-of-Refraction Lower Plenum Experiment, INL/EXT-07-13539.

McIlroy, H.M., 2011, "Matched Index-of-Refraction MH-GTR Prismatic Block Bypass Flow Control and Measurement Plan", INL Internal Report, PLN-3669.

Meinhart, C. D., S. T. Wereley, and J. G. Santiago, "A PIV algorithm for estimating time-averaged velocity fields," Journal of Fluids Engineering-Transactions of the Asme, 2000, Vol. 122 No. 2, pp. 285-289.

Melese, G., Katz, R., 1984, "Thermal and Flow Design of Helium-Cooled Reactors," American Nuclear Society, La Grange Par, IL.

Munson, B., Young, D., Okiishi, T., 2004, A Brief Introduction to Fluid Mechanics, Wiley, Ed. 3, pp. 312.

Nobach, H. and C. Tropea, 2005, "Improvements to PIV image analysis by recognizing the velocity gradients," Experiments in Fluids, Vol. 39 No. 3, pp. 612-620.

Nogueira, J., A. Lecuona, and P. A. Rodriguez, 1999, "Local field correction PIV: on the increase of accuracy of digital PIV systems," Exp Fluids, Vol. 27, pp. 107-116.

Pereira, F., et al. 2004, "Adaptive multi-frame PIV. in 12th international symposium on applications of laser techniques to fluid mechanics," Lisbon, Portugal, July.

Persoons, T., O'Donovan, T. S. and Murray, D. B, 2008, "Improving the measurement accuracy of PIV in a synthetic jet flow," 14th Int. Symposium on Applications of Laser Techniques to Fluid Mechanics, Lisbon, July 2008.

PIV Workshop, 2007, "Particle Image Velocimetry: Theory, Practice and Application," University of Maryland, May 22 and 23, 2007.

PIV, www.piv-online.org

PLN-3669, 2012, "Matched Index of Refraction MHTGR Prismatic Block Bypass Flow Control and Measurement Plan,” Revision 1, Idaho National Laboratory, February 2012.

Raffel, M., C. Willert, and J. Kompenhans, 1998, Particle Image Velocimetry: A Practicle Guide, 3rd ed., Springer.

Satelli, A., Tarntola, S., Campolongo, F., Ratto, M., 2004, Sensitivity Analysis in Practice, John Wiley \& Sons, ISBN 0-470-87093-1.

Sato, H., Johnson, R., and Schultz, R., 2010, "Computational Fluid Dynamic Analysis of Core Bypass Flow Phenomena in a Prismatic VHTR," Annals of Nuclear Energy, 37, pp. 1172-1185.

Scarano, F. and M. L. Riethmuller, 1999, Iterative multigrid approach in PIV image processing with discrete window offset. Experiments in Fluids, Vol. 26, No. 6, pp. 513-523. 
Scarano, F. and M. L. Riethmuller, 2000, "Advances in iterative multigrid PIV image processing," Experiments in Fluids, Vol. 29, pp. S51-S60.

Scarano, F., 2002, "Iterative image deformation methods in PIV," Measurement Science \& Technology, Vol. 13. No. 1, pp. R1-R19.

Scarano, F., 2003, "Theory of non-isotropic spatial resolution in PIV," Experiments in Fluids, Vol. 35, No. 3, p. 268-277.

Scarano, F., 2004, "Development and assessment of nonisotropic spatial resolution in PIV," Particle Image Velocimetry: Recent Improvements, pp. 97-113 and 426.

SimLab, 2002, Simlab Reference Manual.

Sobol, L.M., 1990, “Quasi-Monte Carlo Methods,” Prog. Nucl. Energy, Vol. 24, pp. 55-61.

Sobol, L.M., 1993, "Sensitivity analysis for non linear mathematical models," Math. Model. Comput. Exp., Vol. 1, pp. 407-414.

Stanislas, M., et al., 2008, "Main results of the third international PIV Challenge," Experiments in Fluids, Vol. 45. p. 27-71.

Stoots, C., S. Becker, K. Condie, F. Durst, D. McEligot, 2001, “A Large Scale Matched Index of Refraction Flow Facility for LDA Studies Around Complex Geometries," Exp. in Fluids, Vol. 30, pp. 391-398.

Suzuki, K., Suzuki, K. Mogi, H., Fumizawa, M., Iyoku, T, Sawa, K., 1985, “Analysis of Core Flow Deviation Caused by the Large Gaps Between Horizontal Block Interfaces," Specialists meeting on Safety and Accident Analysis for Gas-Cooled Reactors, Oak Ridge, TN.

Timmins, B. H., B. W. Wilson, B. L. Smith, and P. Vlachos, 2012, "A method for automatic estimation of instantaneous local uncertainty in particle image velocimetry measurements," Exp Fluids, DOI 10.1007/s00348-012-1341-1, June 2012.

Udrea, D. D., et al., 1996, "Two sub-pixel processing algorithms for high accuracy particle centre estimation in low seeding density particle image velocimetry," Optics and Laser Technology, Vol. 28, No. 5, pp. 389-396.

Ullum, U., J. J. Schmidt, P. S. Larsen, D. R. McCluskey, 1998, "Statistical Analysis and Accuracy of PIV Data," J. of Visualization, Vol. 1, No. 2, pp. 205-216.

Uzol, O., Camci C., 2001, "The Effect of Sample Size, Turbulence Intensity and the Velocity Field on the Experimental Accuracy of Ensemble Averaged PIV Measurements," Proc. of 4th Int. Sym. on Particle Image Velocimetry, Gottingen, Germany.

Wereley, S. T. and C. D. Meinhart, 2001, "Second-order accurate particle image velocimetry," Experiments in Fluids, Vol. 31, No. 3, pp. 258-268.

Westerweel, J., 1997, "Fundamentals of digital particle image velocimetry," Journal of Measurement Science Technology, Vol. 8, 1379-1392.

Westerweel, J., D. Dabiri, and M. Gharib, 1997, "The effect of a discrete window offset on the accuracy of cross-correlation analysis of digital PIV recordings," Experiments in Fluids, Vol. 23, No. 1, pp. 20-28.

Westerweel, J., P. F. Geelhoed, and R. Lindken, 2004, "Single-pixel resolution ensemble correlation for micro-PIV applications," Experiments in Fluids, Vol. 37, No. 3, pp. 375-384.

Wieneke, B., 2005, "Stereo-PIV using self-calibration on particle images," Experiments in Fluids, Vol. 39, No. 2, pp. 267-280.

Willert, C. E. and M. Gharib, 1991, "Digital Particle Image Velocimetry," Experiments in Fluids, Vol. 10, No. 4, pp. 181-193. 
Wilson, B., Smith, B., 2011, "Errors in Mean and Fluctuating Velocity Due to PIV Bias and Precision Uncertainties," NURETH-14, Toronto, Canada.

Wilson, Brandon M., 2009, "Demonstration of a Completely Described Swirling Jet Experiment Used for Numerical Validation," All Graduate Theses and Dissertations, Paper 454.

Yoon, S., Cho, Y., Kim, K., Kim, M., Lee, W., and Park, G., 2007, "Experimental Evaluation of the Bypass Flow in the VHTR Core," SMiRT-19, Toronto. 


\section{Appendix A}

\section{MATLAB Script for MIR Mapping}




\section{Appendix A MATLAB Script for MIR Mapping}

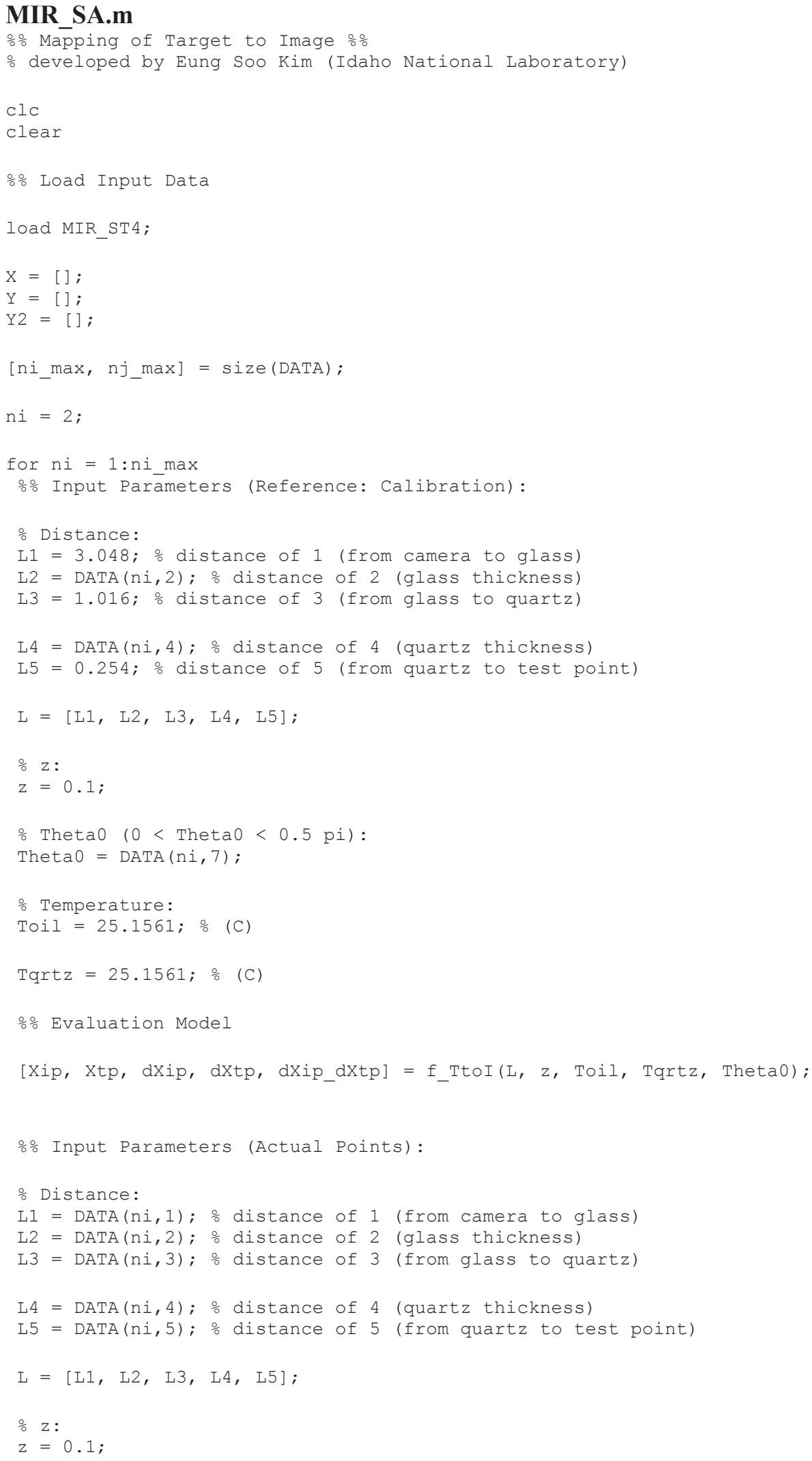




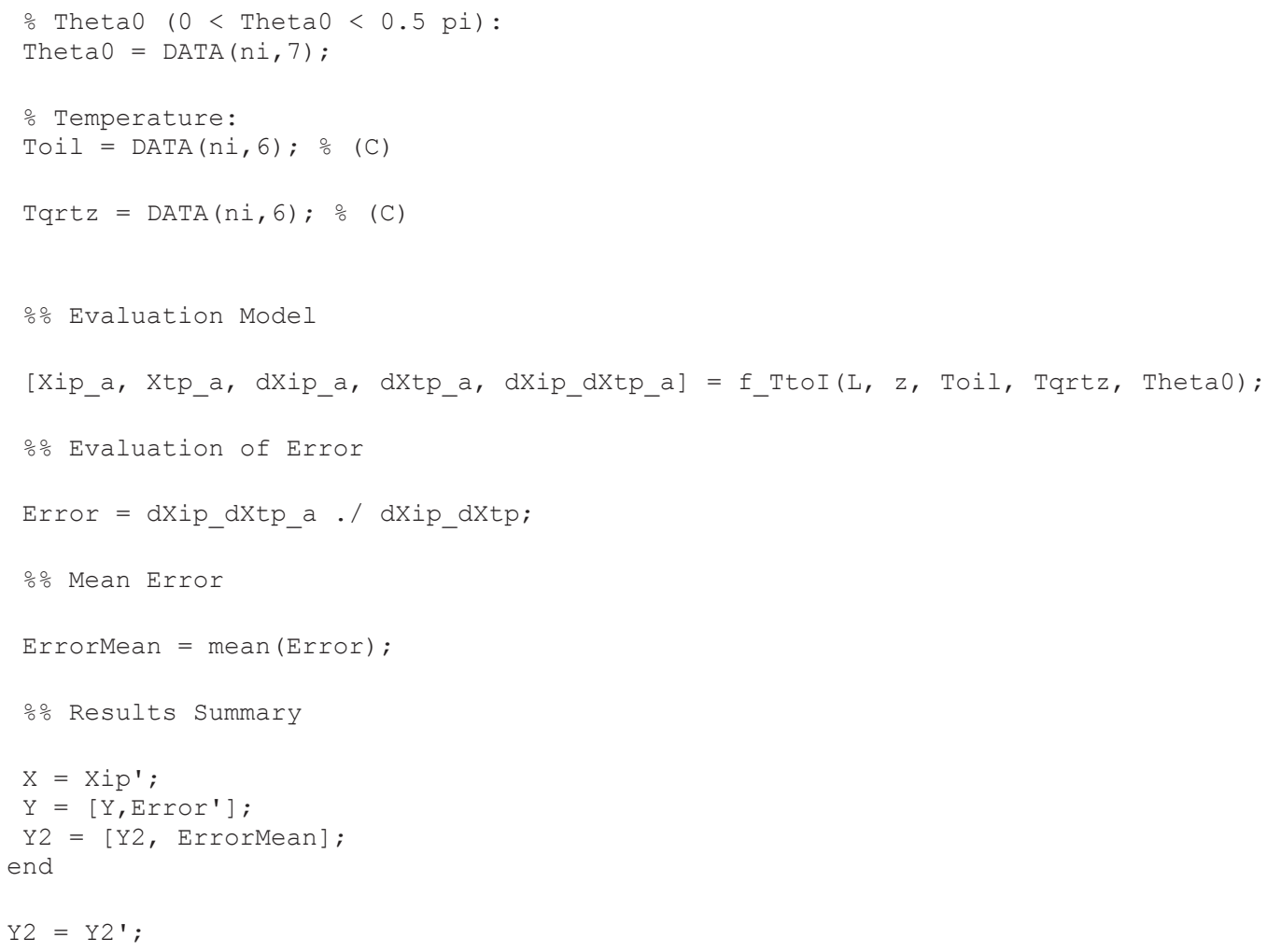

\section{F_TtoI.m}

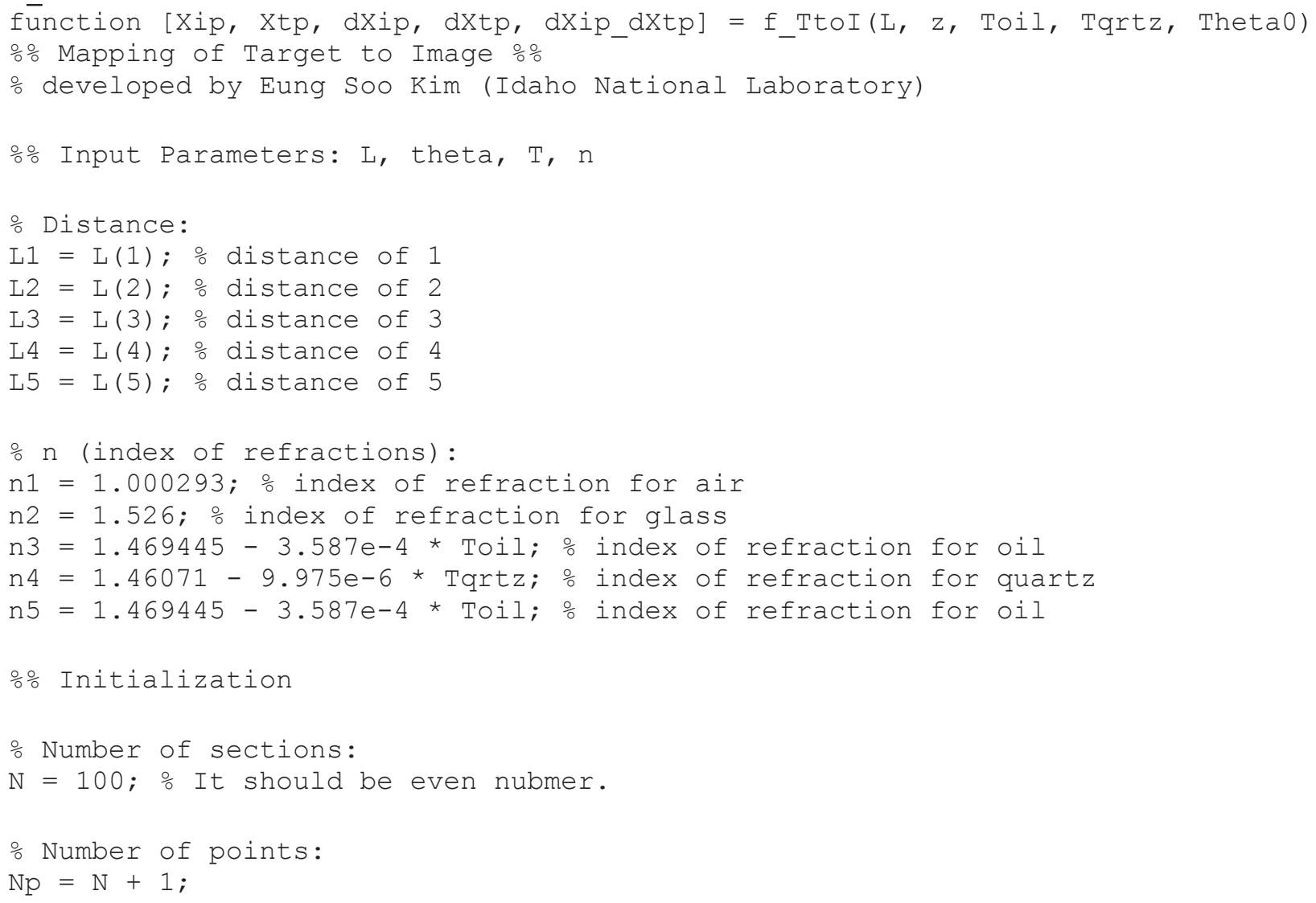




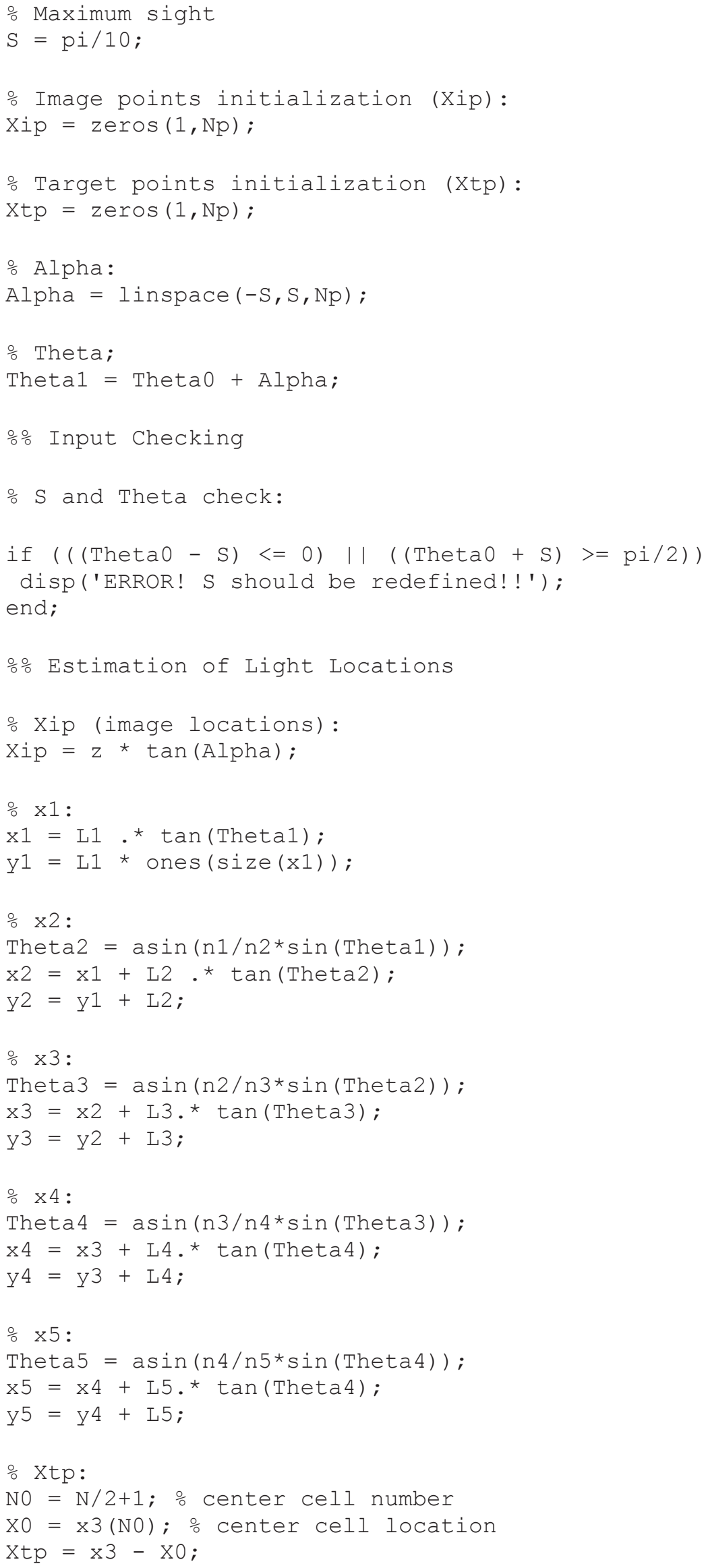




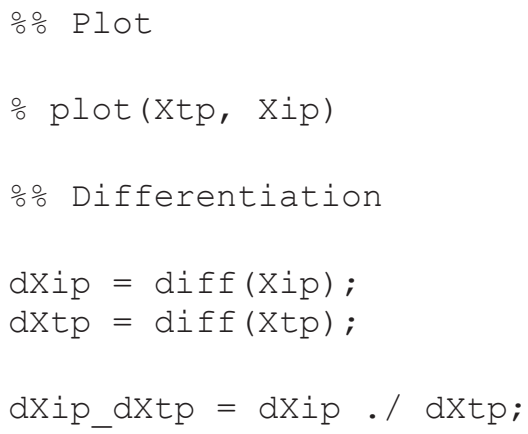


Appendix B

\section{Uncertainty Quantification Methodology For MIR Facility}




\title{
Appendix B \\ Development of an Uncertainty Quantification Method for MIR Facility
}

\author{
Barton Smith, Utah State \\ University \\ Pavlos Vlachos, Virginia Tech \\ University
}

\section{INTRODUCTION}

Particle Image Velocimetry (PIV) is a non-invasive, quantitative, flow visualization tool developed to measure fluid velocities over a wide range of length and time scales. The technique typically employs micron-size flow tracer particles, which are illuminated by a pulsed laser and imaged with a high-frequency camera. Processing algorithms are then used to determine the displacement of particle patterns within an image sequence and to estimate the velocity field (Willert and Gharib 1991). An overview of the development of DPIV over the first 20 years of the method is given by Adrian (Adrian 2005).

PIV was first developed in the 1980s, and the initial work of Meynart (R 1983) was followed by numerous seminal contributions that established the foundations of the method (Yao and Adrian 1984, Adrian and Yao 1985, Keane and Adrian 1990a, Adrian 1991). The introduction of digital image acquisition (Willert and Gharib 1991) (DPIV) provided a transformative evolution of the method and triggered its widespread use and an explosive growth of applications. Refinements over the next 20 years improved robustness and accuracy of the technique, including the development of stereoscopic (3-component) planar PIV (Soloff, Adrian et al. 1997, Willert 1997), iterative, and adaptive methods (Wereley and Meinhart 2001, Scarano 2002, Scarano 2003, Wereley and Gui 2003, Scarano 2004). A comprehensive history of these improvements can be traced through early reviews (Adrian 1991, Grant 1997) and more recent sources (Stanislas, Kompenhans et al. 2000, Adrian 2005, Kompenhans, Raffel et al. 2007, Adrian and Westerweel 2010). Currently, the term PIV is used to encompass the extensive family of methods that are based on evaluating the particle patterns displacement using statistical cross- correlation of consecutive images with high number density of flow tracers (Adrian and Westerweel 2010).

However, the development of PIV methods did not involve simultaneous rigorous quantification of measurement uncertainty. As a result, despite the numerous applications, theory, and contributions, there is currently no widely accepted framework for reliable quantification of PIV measurement uncertainty. The situation is exacerbated by the fact that PIV measurements involve instrument and algorithm chains with coupled uncertainty sources, rendering quantification of uncertainty far more complex than most measurement techniques. Consequently PIV results are often received with skepticism. Therefore developing a fundamental methodology for quantifying the uncertainty for PIV is an important and outstanding challenge.

The first attempt to tackle this problem employed an "error-surface" methodology which would be constructed by mapping the effects of selected primary error sources such as shear, displacement, seeding density, and particle diameter to the true error for a given measurement (Timmins, Wilson et al. 2012). This approach is roughly analogous to a more traditional instrument calibration procedure for 
standard experimental instruments. The generated error surface provides the means to associate the corresponding distribution of errors to any combination of inputs of the error sources within their parameters space, as quantified directly from the actual experiment. Ultimately in order to comprehensively quantify the uncertainty, all possible combinations of displacements, shears, rotations, particle diameters, and any other parameter used must be exhaustively tested which can make this method computationally expensive. Moreover, many of the relevant parameters may not be easily obtained from a real experiment.

Sciacchitano et al. proposed a method to quantify the uncertainty of PIV measurement based on image matching (Sciacchitano, Wieneke et al. 2013). The uncertainty of measured displacement is calculated from the ensemble of disparity vectors, which are due to incomplete match between particles, within the interrogation window. This method accounts for random and systematic errors, however peak-locking errors and truncation errors cannot be detected. Moreover to calculate the instantaneous local uncertainty, researchers need to do particle image pair detection and image matching for every single interrogation spot which makes this method also computationally expensive.

\section{UNCERTAINTY QUANTIFICATION METHODS FOR PIV}

When best practices are followed, much of the uncertainty in a PIV measurement can come from the computation of the individual displacements at each interrogation window. Two methods have been developed for determining this fundamental PIV uncertainty. The Uncertainty Surface Method (USM) is a response surface method developed at USU, while the Signal to Noise Ratio Method (SNR) was developed at Virginia Tech. These are now described.

\subsection{Uncertainty Surface Method}

The USM was first reported in Timmins 2010. Under the present work, the method has been refined, written into user-friendly Matlab codes, and documented. In order to employ the Uncertainty Surface Method, one must choose the several measureable parameters that one assumes impact the error in a PIV computation. In our work, based on work published earlier and discussed by Adrian and Westerweel 2010, we chose particle image diameter, particle image density, particle displacement, and shear.

Since the uncertainty of a PIV calculation depends on the algorithm used (meaning the software and all settings), the response of the algorithm to varying degrees of each of the contributors must be systematically tested to generate an Uncertainty Surface for that algorithm. Synthetic images are used for this purpose.

Once a surface has been generated, the uncertainty of each vector may be determined by measuring the particle image diameter, particle image density, particle displacement, and shear, and then querying the surface based on those results. Obviously, the method requires a sensible choice in parameters. If other parameters affect the uncertainty and are not included in the surface, a poor estimate of uncertainty can result. Some relevant parameters, such as out-of- plane motion, can be difficult to measure, making them difficult to include in the USM.

Matlab-based software to generate synthetic images, generate the uncertainty surface, and compute uncertainty for 2C and 3C PIV data, are available on the USU EFDL website (http://efdl.neng.usu.edu/EFDL/Code.html). 


\subsubsection{Objective}

The aim of the USM is to produce an uncertainty band of the instantaneous $u$ and $v$ velocity at each measurement location that contains the true velocity value to $95 \%$ probability. Over an ensemble, these values can be used to form the uncertainty of the mean velocity and the Reynolds stresses, as discussed below.

\subsubsection{Detail Description of the Methodology}

For a specific PIV algorithm (meaning software and its settings), a series of tests are used to determine the uncertainty response of the algorithm to particle displacement, particle image diameter, particle image density, and shear. The tests consist of computing vector fields from a large series of synthetic images that are based on flows with varying displacement and shear. The images also represent a range of particle image density and particle image diameter. By comparing the resultant vector fields to the known displacement for each case, the distribution of error, or the uncertainty for a specific combination of the four parameters can be determined. The four-dimensional uncertainty response is termed the "uncertainty surface." This process must be performed for each algorithm of interest, but the resultant uncertainty surface is used for all future calculations.

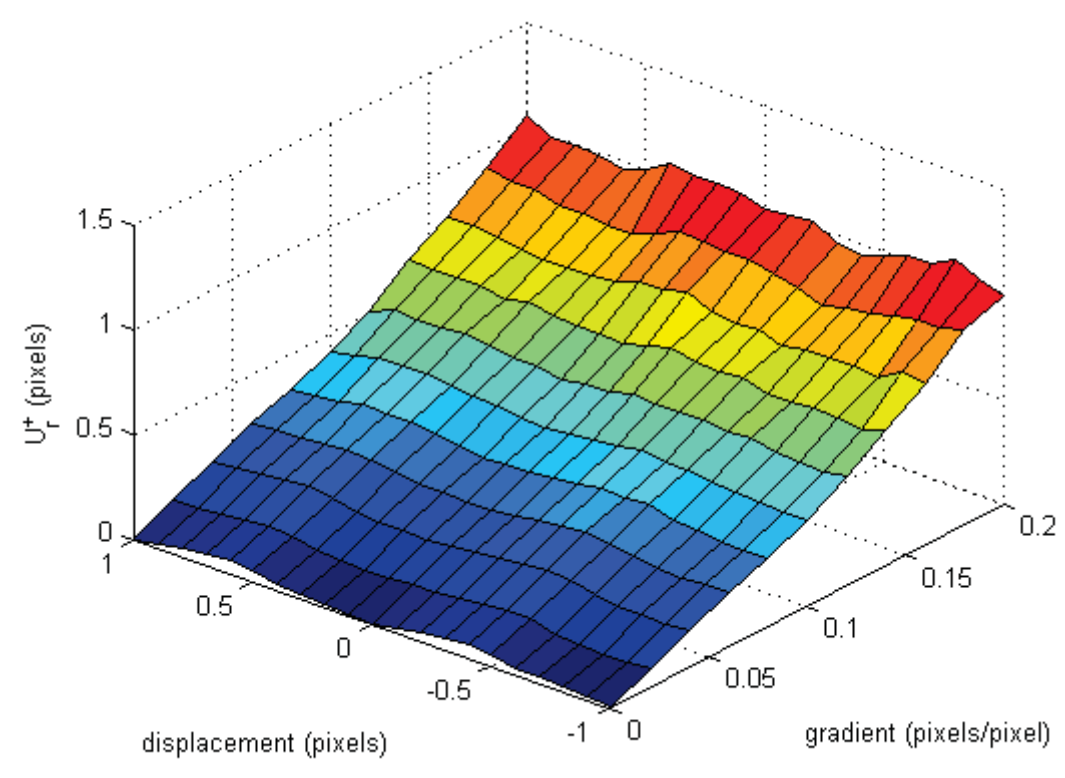

Figure 1: Surface of $U_{r}{ }^{+}$for 2.50 pixel particle image diameter and 0.0195 particles/pixels ${ }^{2}$ density.

Once a PIV dataset has been processed to find the velocity field, the values of the four error sources are determined from the raw image data and the velocity vectors. Then, the uncertainty surface is queried for the value of the uncertainty for each instantaneous vector. Each component of velocity is assigned three instantaneous uncertainties by the uncertainty surface: a systematic uncertainty $b_{k}$, and an upper and lower random uncertainty $\left(r_{\text {low }}\right.$ and $\left.r_{\text {high }}\right)$, as shown in Figure 2. 


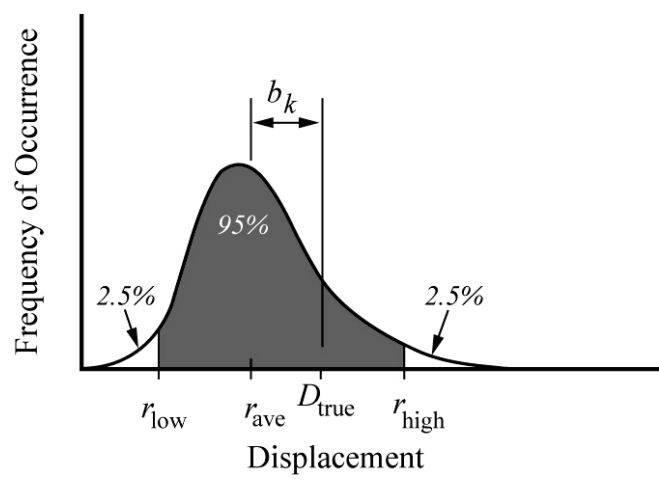

Figure 2: Probability distribution of error and definition of bias and random uncertainties.

Of the four parameters chosen, particle image density is the most challenging to measure. An autocorrelation-based density (ABD) method for estimating the particle image density was developed as part of the present work (Warner and Smith 2013). An empirical relationship between the autocorrelation peak height and the particle image density, particle image diameter, average particle intensity, and interrogation region size was established using synthetic images. Simulated camera noise was added to synthetic images and was found to have insignificant impact.

Experimental validation of the synthetic image results was performed using two distinct experimental setups. The setups consisted of a rectangular jet seeded with olive-oil droplets in air and a water-filled aquarium seeded with hollow glass spheres. Images with varying particle image densities were acquired from both setups and analyzed using the ABD method. The results from both cases show a linear relationship between the mass added and the estimated particle image density. A comparison between the ABD method and the local maximum method was made. Both methods provided similar results for lower seed density, but the local maximum method became less reliable as the amount of overlapping particles increased.

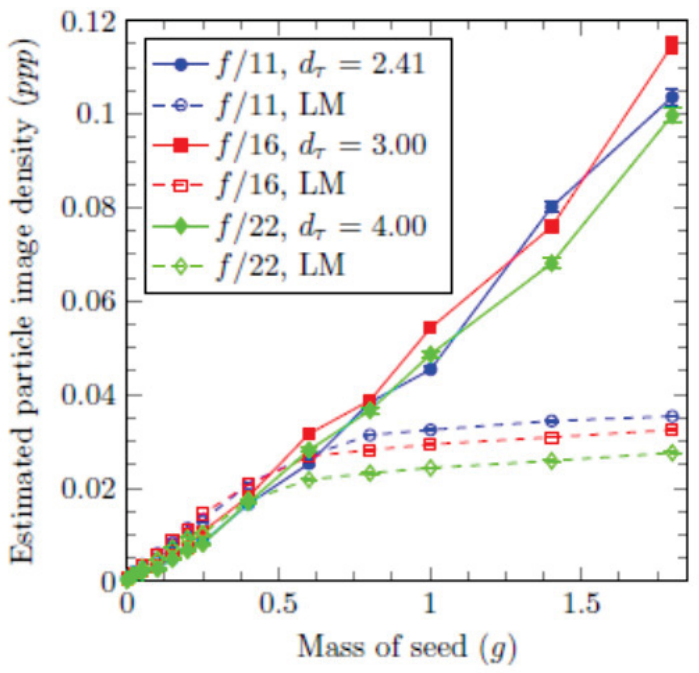

(a)

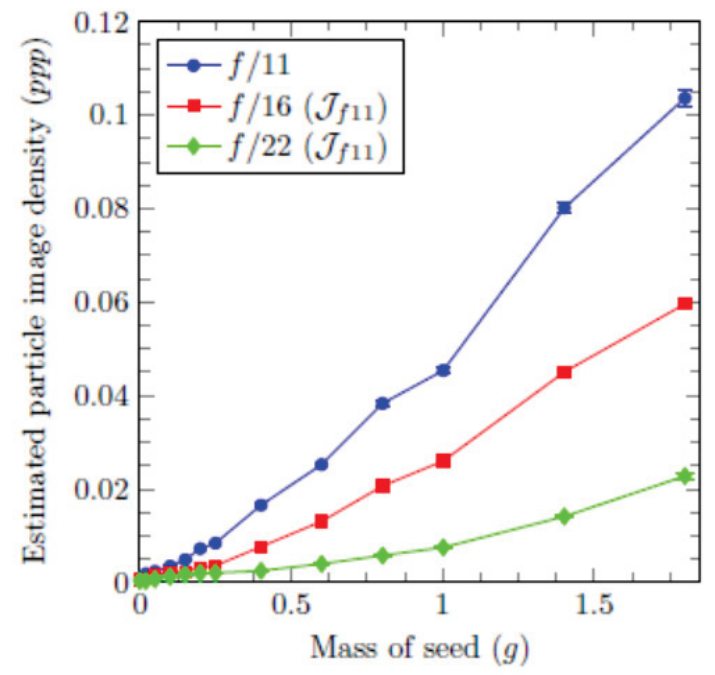

(b)

Figure 3: Estimated seeding density as a function of the total mass of seed particles added to the water. Values are shown using (a) three different particle image diameters and (b) three different image intensities. The solid lines with the solid symbols represent the average particle image density calculated using the ABD method. The dashed and dotted lines with the open symbols represent the average particle image density estimates from the LM method. Results obtained using $128 \times 128$ pixel interrogation region. 
The slope of the linear density relationship was found to be a function of the level of light incident on the camera sensor. A decreased level of intensity incident on the camera sensor, whether due to decreasing the laser intensity or aperture size of the lens, decreases the number of visible particles images and decreases the overall estimate of the particle image density.

The present results were for PIV setups, for which only the particle image density is important. However, the ABD method should work for any image with diffraction-limited particles. It may also be a feasible method for determining actual particle density (as opposed to image density) with additional pre-processing of the images and calibration.

8-bit

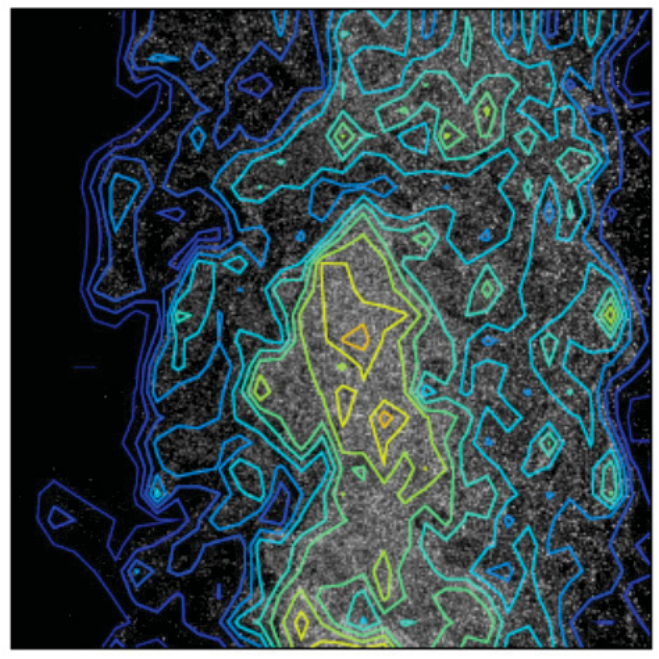

14-bit

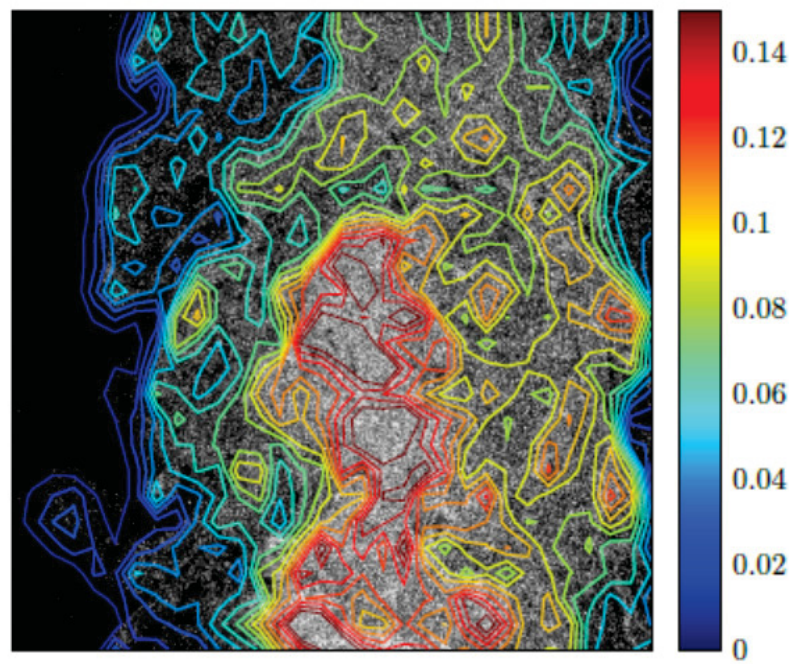

Figure 4: A PIV image and the corresponding seeding density based on the ABD method (overlaid contours) for images acquired simultaneously from two cameras with differing bit depth. The color bar shows the particle image density in particles/pixel. Images are used with permission from Sandia National Laboratory.

\subsubsection{Experimental Validation}

To date, validation of the USM has been performed based on velocity statistics derived from a PIV measurement compared to hot-wire anemometer measurements. Comparisons based on instantaneous data rather than statics will be made in the near future. The flow field of a planar jet was measured with PIV and hot-wire anemometry. The effects of PIV uncertainty on the time-average velocity and the Reynolds normal stresses, were also assessed.

Random uncertainty from these four sources affected the rate at which converged statistics were acquired. These errors are two-sided and, when sufficient data are obtained, a converged mean that agrees with the hot-wire solution was measured. However, the measurement noise increased the Reynolds normal stress reported by the PIV measurement system resulting in Reynolds normal stress levels that were overestimated. Of the four error sources, velocity gradients were the largest contributor to PIV random uncertainty.

For pixel displacements of $\otimes x \sim d \tau$, the measured mean velocity was strongly biased, while Reynolds normal stresses were significantly overestimated for $\otimes x \sim 4 d \tau$. This is due to a combination of decreased dynamic range for the smaller pixel displacements and sub-pixel interpolation uncertainties. 


$\triangle$ PRANA $\quad$ DaVis Hot Wire

0.5 pixels

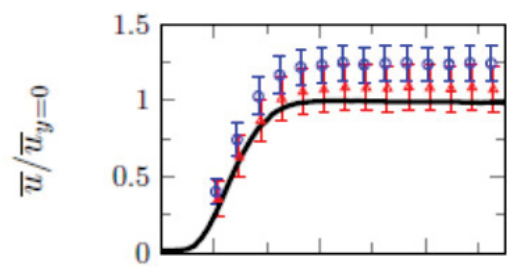

1.1 pixels

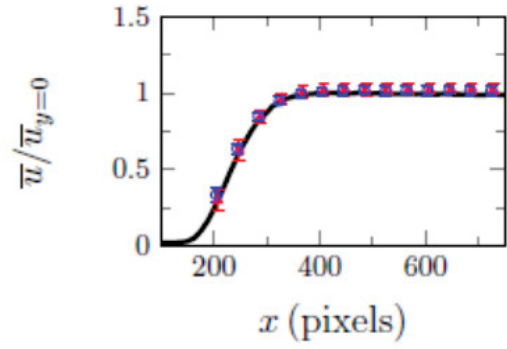

0.7 pixels

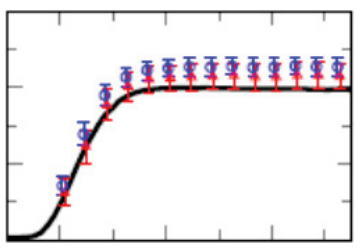

2.1 pixels

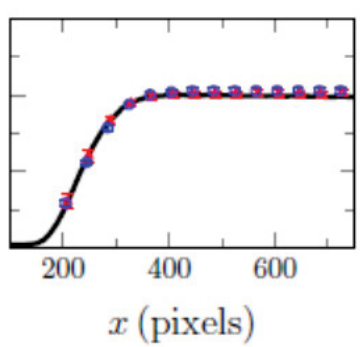

0.9 pixels

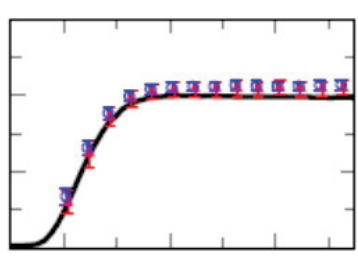

3.2 pixels

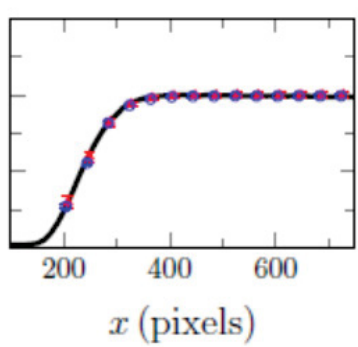

Figure 5: Profiles of the time-averaged velocity magnitude, $u$, using the hot wire and PIV algorithms (DaVis and PRANA) with $16 \times 16$ pixel interrogation regions for varying small displacements.

The work presented did not account for through-plane motion, and some evidence exists as random through-plane motion increases, the performance of the method degrades. In principle, the uncertainty surface method could be extended to include these effects. In fact, the VT team has developed a method to measure through-plane motion that is described below.

\subsubsection{Current Status and Future Work}

The USM using four parameters (particle image size, particle image density, particle displacement and shear) is complete. In the near future, its results will be compared to those of the SNR and other methods. The primary weakness of USM is parameters that generate error but are not included in the surface. Primary among these is likely to be through-plane motion, which caused a loss of correlation and can also generate errors to changes in particle illumination as particles pass through the Gaussian-shaped laser sheet (Nobach 2009). While this parameter is widely held to be important, it has not yet been included in the uncertainty surface since a method to measure through-plane motion with a single camera has yet to be developed. Mutual Information (MI), discussed in Section 5.2.3.3 below, has the potential to provide information about through-plane motions. Future work on the USM could include using MI as a $5^{\text {th }}$ parameter, or, as a replacement for one of the currently used parameters.

\subsection{Signal to Noise Ratio Uncertainty Method}

\subsubsection{Objective}

In particle image velocimetry (PIV) the measurement signal is contained in the recorded intensity of the particle image pattern superimposed on a variety of noise sources. The inherent amount of signal mutual information between consecutive images governs the strength of the resulting PIV cross correlation and ultimately the accuracy and uncertainty of the produced PIV measurements. Hence we posit that correlation signal-to-noise-ratio (SNR) metrics calculated from the correlation plane can be 
used to quantify the quality of the correlation and the resulting uncertainty of an individual measurement. In this paper we present a framework for evaluating the correlation SNR using a set of different metrics, which in turn are used to develop models for uncertainty estimation. A new SNR metric termed "mutual information" (MI) which quantifies the amount of common information (particle pattern) between two consecutive images is also introduced and investigated. This measure provides a direct estimation of the apparent $\mathrm{N}_{\mathrm{I}} \mathrm{F}_{\mathrm{I}} \mathrm{F}_{\text {O }}$ parameter of an image pair providing an alternative approach towards uncertainty estimation but also connecting the current development to one of the most fundamental principles of PIV and the previously established theory. The SNR metrics and corresponding models presented herein are expanded to be applicable to both standard and filtered correlations and the notion of "valid" measurement is redefined with respect to the correlation peak width. These advancements lead to more robust uncertainty estimation models, which are tested against both synthetic benchmark data as well as actual experimental measurements. For all cases considered here, expanded uncertainties are estimated at the $95 \%$ confidence level, and the resulting calculated coverages are approximately $95 \%$ thus demonstrating the feasibility and applicability of these new models for direct estimation of uncertainty for individual PIV measurements.

\subsubsection{Detail Description of the methodology}

In this work we adopt an alternative approach and we seek to quantify the PIV displacement uncertainty directly from the information contained within the cross-correlation plane. The cross- correlation plane represents the distribution of probabilities of all possible particle image pattern displacements between consecutive frames, combined with the effect of the number of particles, the mean particle diameter and effects that contribute to loss of correlation. As described by Adrian and Westerweel (Adrian and Westerweel 2011) (p. 322): "The height of the peak is proportional to the image density $N_{I}$, the out of plane loss of correlation $F_{O}$ and the in-plane loss of correlation $F_{I}$. The shape of the peak is determined by the convolution of the particle image self-correlation with the displacement distribution in the measurement volume. "In other words, the correlation plane is a surrogate of the combined effects of the various sources of error that govern the accurate estimation of a particle pattern displacement. Hence, in this work we will seek to establish appropriate measures that quantify the cross-correlation quality by means of signal-to-noise ratio (SNR) and establish the relationship of these metrics to the individual measurement uncertainty.

One measure of the cross-correlation SNR is the primary peak ratio (PPR), namely the ratio between the highest correlation peak to the second tallest peak as shown in Figure 6. In early PIV papers, PPR was used as a measure of the detectability of the true displacement (Keane and Adrian 1990b, Keane and Adrian 1991). A measurement would be considered as valid if PPR were higher than a user defined threshold (often 1.2), or the measurement is rejected if PPR is smaller than that value. Based on this criterion, it was established that the product of $\mathrm{N}_{\mathrm{I}} \mathrm{F}_{\mathrm{I}} \mathrm{F}_{\mathrm{O}}$ would determine the probability of getting a valid detection and in order to get a $95 \%$ probability of valid detection, the minimum $\mathrm{N}_{\mathrm{I}} \mathrm{F}_{\mathrm{I}} \mathrm{F}_{\mathrm{O}}$ value should be approximately 5 (Keane and Adrian 1992). Unfortunately, the effects of in-plane and out-of-plane loss of correlation are difficult to quantify in real experiment, thus making $\mathrm{N}_{I} \mathrm{~F}_{\mathrm{I}} \mathrm{F}_{\mathrm{O}}$ difficult to estimate in real experiment cases. However this establishes a clear relationship between a measure of the correlation strength (PPR) and number of correlated particle image pairs.

The PPR value is easy to compute and provides a practical measure of the quality of a cross- correlation. Hain and Kahler (Hain and Kahler 2007) suggest that a threshold PPR value of about 2 can reliably avoid spurious vectors, and based on this they proposed a scheme for the optimal selection of cross-correlations across a range of interframe time delays. Similarly for extending the PIV velocity dynamic range using multiple pulse separation imaging, Persoons and O'Donovan used a weighted peak ratio value as a criterion to calculate the optimum pulse separation (Persoons and O'Donovan 2010). 
Recently, Charonko and Vlachos proposed an uncertainty quantification method based on PPR (Charonko and Vlachos 2013). The relationship between the distribution of velocity error and PPR value was studied and a model for calculating the uncertainty based on the PPR value of a given measurement was developed. Using this method, the uncertainty of PIV measurement can be predicted without the a-priori knowledge of image quality and local flow condition. Reliable uncertainty estimation results using a phase-filtered correlation (Eckstein, Charonko et al. 2008, Eckstein and Vlachos 2009) were shown. However for standard cross-correlation techniques, the uncertainty estimation provided by this method is not as good. This was attributed to the insufficient treatment of noise effects inherent in the standard cross-correlation.

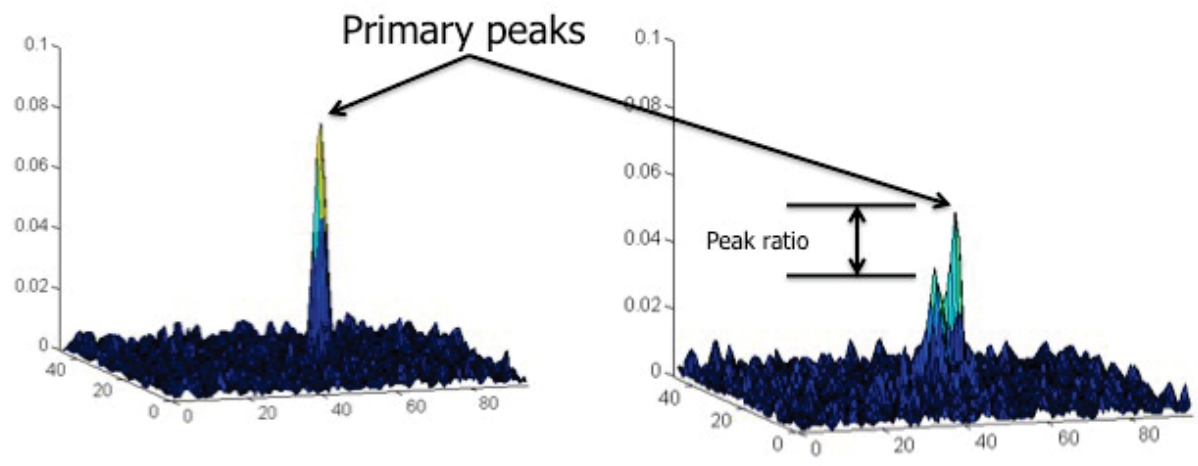

Figure 6: Left: Single cross-correlation peak with high probability of accurate detection. Right: Two primary peaks in the correlation plane. The closer the peak heights are with respect to each other, the lower the probability of accurate detection.

Beyond the PPR other metrics exist for quantifying the cross-correlation SNR. Kumar and Hassebrook defined several signal to noise ratios of the correlation related to peak detectability, namely peak ratio (PPR), peak-to-root mean square ratio (PRMSR), and peak-to-correlation energy (PCE) (Kumar and Hassebrook 1990). All three of these metrics measure the strength of correlation but the PPR is a mostly heuristic parameter while in contrast the PCE and PRMSR are more fundamental routed to signal processing theory (Kumar and Hassebrook 1990). However, within the scope of PIV methods, neither PCE nor PRMSR have been considered.

In this work, we will extend the original work by Charonko and Vlachos (Charonko and Vlachos 2013) to calculate cross-correlation SNR metrics using only the information from the correlation plane to develop models for uncertainty estimation. Here, in addition to the PPR, we will consider PRMSR and PCE and cross-correlation entropy (based on information entropy ([Shannon 2001]) and we expand the previous work to make these measures applicable to both standard and phase filtered cross-correlation. More importantly, we develop a new metric we term "mutual information" (MI) that we hypothesize provides a direct estimation of the apparent $\mathrm{N}_{\mathrm{I}} \mathrm{F}_{\mathrm{I}} \mathrm{F}_{\mathrm{O}}$ for each image pair. This metric also will be used to develop a model for uncertainty estimation but in contrast to the other models that are adopted from the generic signal processing literature; MI directly connects to the fundamental PIV principles.

Details of the definition and calculation of these metrics will be provided in the following section. The relationship between velocity error distribution or standard uncertainty and each metric are obtained by statistical analysis. The functions used to quantify each relationship are calculated from curve fittings.

\subsubsection{Background and Methodology}

\subsubsection{Correlation Plane Signal to Noise Ratio (SNR)}

The random correlation peaks distributed along the correlation plane correspond to correlations between distinct particle image pairs. A valid displacement measurement is achieved when the highest detectable peak in the plane represents the true displacement. As a result, the strength (detectability) of 
the primary peak with respect to surrounding peaks represents the signal to noise ratio of the correlation plane. Hence following the work by Kumar and Hassebrook (Kumar and Hassebrook 1990), measures of the correlation SNR can be defined and are shown in Table 1. These include the PPR, PRMSR, and PCE. Table 1 also provides the definitions of these filters and a one-dimensional graphical representation.

Table 1: Definition, separate parts and 1-D example of PPR, PRMSR and PCE.

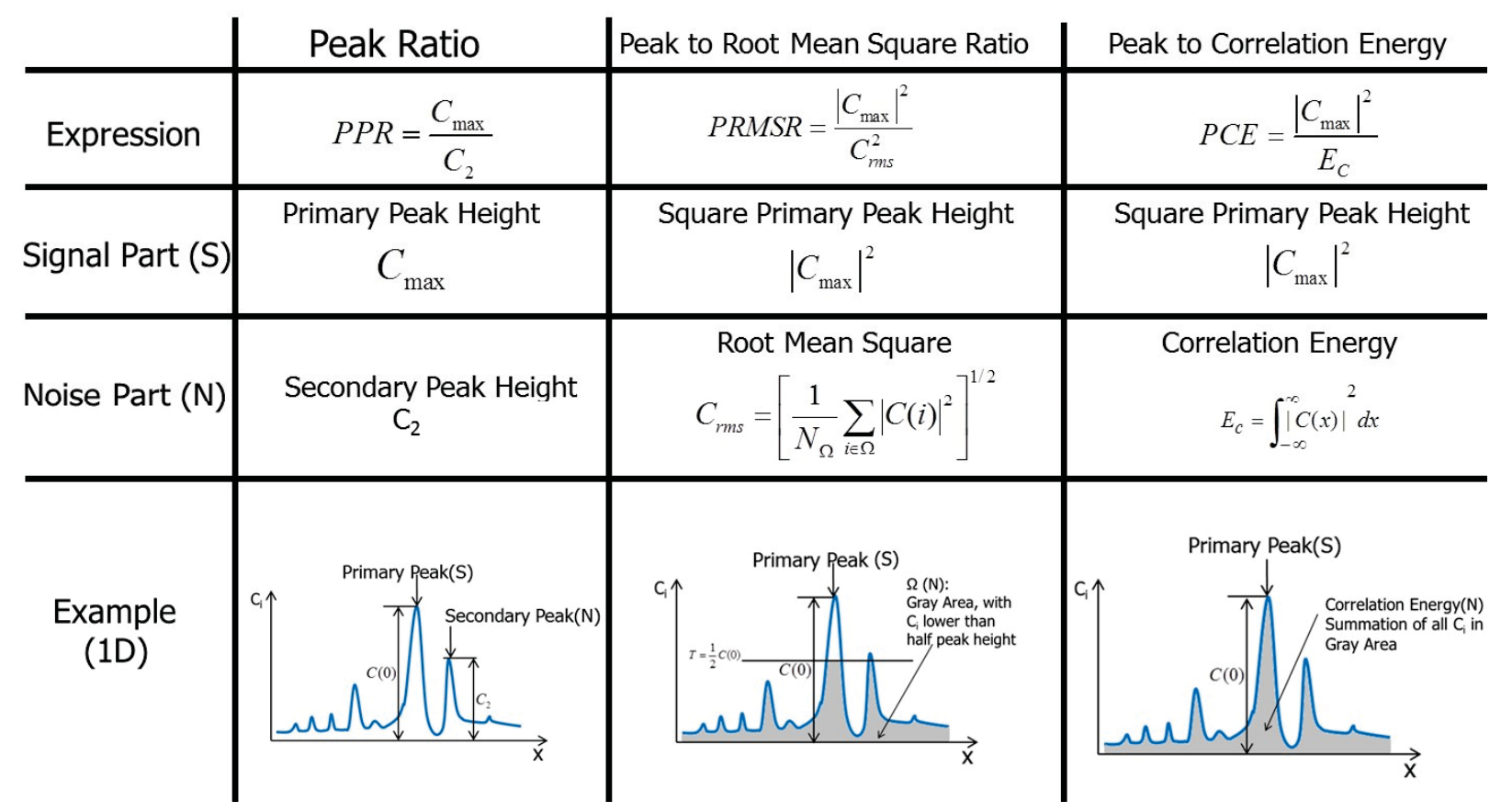

Effectively all three of these metrics measure the detectability of the primary peak with respect to alternative correlations. However, in contrast to the PPR which is an ad-hoc metric, the PRMRS and PCE are amenable to analytical derivation if the signal statistical properties are known (Kumar and Hassebrook 1990), hence they offer the potential for developing a corresponding theoretical foundation for the uncertainty estimation. This aspect however will not be pursued during this work.

Another signal to noise ratio measure considered herein is the cross-correlation entropy or information entropy (Shannon 2001). This is based on the notion that if perfect matching between two image patterns exists in the absence of any noise, the correlation will yield a single sharp peak and the correlation entropy will be minimal. As more random correlations exist the entropy would increase. To calculate the entropy of the cross correlation plane, we first construct the histogram of the correlation plane based on the correlation value of every point on the plane. In our work, we use 30 bins to build the histogram. After the histogram is made, the probability of finding one point in a certain bin is calculated as:

$$
p_{i}=\frac{\# \text { of points } @ \text { bin } i}{\text { Total \# points of whole plane }}
$$

Then the entropy of the cross correlation plane was calculated as:

$$
\text { Entropy }=\sum_{i=1}^{30} p_{i} \log 1 / p_{i}=-\sum_{i=1}^{30} p_{i} \log p_{i}
$$




\subsubsection{Role of Image Background Noise on Correlation SNR}

The information about the true displacement in the correlation plane is contained in the correlation of the fluctuating intensities. If the correlation is written as:

$R(s, t)=\mathrm{R}_{C}(s, t)+R F(s, t)+R D(s, t)$

where the overall correlation plane is decomposed into $\mathrm{R}_{\mathrm{C}} \mathrm{R}_{\mathrm{F}}$ and $\mathrm{R}_{\mathrm{D}}$ which are respectively the correlation of the mean background intensity over the interrogation windows, the correlation of the background noise in one window with the fluctuating intensity in the other window, the cross correlation of the fluctuating image intensities. It is common practice to subtract the image mean intensity before preforming a cross-correlation, which would effectively remove all contributions from the background and only provide $\mathrm{R}_{\mathrm{D}}$. However in practice this does not always hold true due to various illumination effects and imaging distortions. Although for the estimation of the true displacement such residuals would have negligible effect, in contrast for the calculation of the correlation SNR they can profoundly affect the metrics. In the work by Charonko and Vlachos (Charonko and Vlachos 2013) the standard correlation which was subject to this effect performed inferiorly to the phase filter correlation which in turn is largely immune to such effects. Hence in order to address this limitation and provide more robust estimation of the different correlation metrics we propose that it is appropriate to subtract the minimum value of the correlation plane. This is demonstrated below.

Figure $7 \mathrm{a}$ and $\mathrm{b}$ shows an example of a particle image with and without background noise. The cross correlation plane of these two image sets are shown in Figure 8a and $\mathrm{b}$. The minimum correlation value of the cross correlation plane is on the order of $10^{6}$. After we subtract the correlation plane of Figure $8 b\left(R_{D}\right)$ from Figure $8 \mathrm{a}(\mathrm{R})$, the left plane Figure 8c can be considered as the correlation related to background image noise, the $\mathrm{R}_{N}$ term. The mean value of this plane is also close to $10^{6}$. Subtraction of the minimum correlation value from the correlation plane effectively eliminates the effect of background image noise on the cross correlation plane.

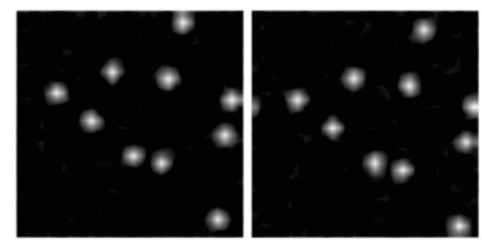

(a)

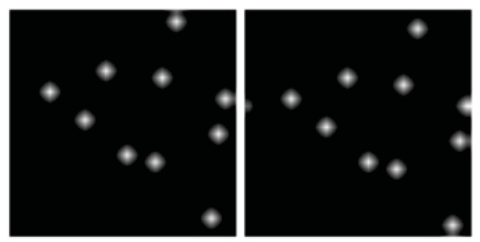

(b)

Figure 7: Particle image sets examples (a) with background noise; (b) same particle images without background noise

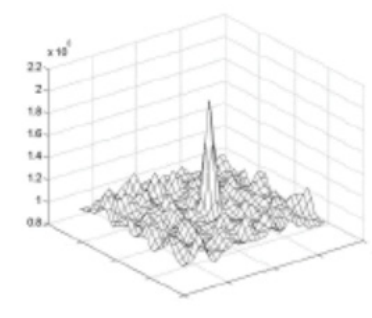

(a)

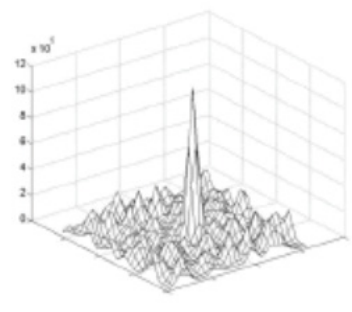

(b)

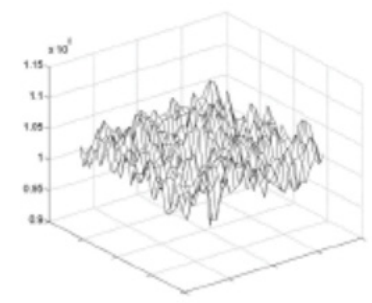

(c)

Figure 8: (a) cross correlation plane of particle images with background noise (b) cross correlation plane of particle images without background noise (c) the correlation plane related to background noise 


\subsubsection{Mutual Information (MI) and relationship to NIFIFO}

As discussed earlier, the correlation peak height is proportional to the product of the image density $\mathrm{N}_{\mathrm{I}}$, the out of plane loss of correlation $\mathrm{F}_{\mathrm{O}}$ and the in-plane loss of correlation $\mathrm{F}_{\mathrm{I}}$, and the shape of the peak is determined by the particle image self-correlation and displacement distribution. Based on this insight we introduce a new measure of the correlation SNR, which we term Mutual Information (MI). MI is defined as:

\section{$\frac{\text { Contribution of all correlated particle pairs }}{\text { Contribution of one correlated particle pair }}=$ Total number of correlated particle pairs $=$ Mutual information}

MI provides the means to directly calculate the $\mathrm{N}_{\mathrm{I}} \mathrm{F}_{\mathrm{I}} \mathrm{F}_{\mathrm{O}}$ from the information contained within the correlation plane. The calculation of the MI is based on dividing the peak magnitude of the cross-correlation by the autocorrelation of the "mean" particle as measured by the diameter of the image autocorrelation. A schematic of the calculation flow chart is shown below (Figure 9).
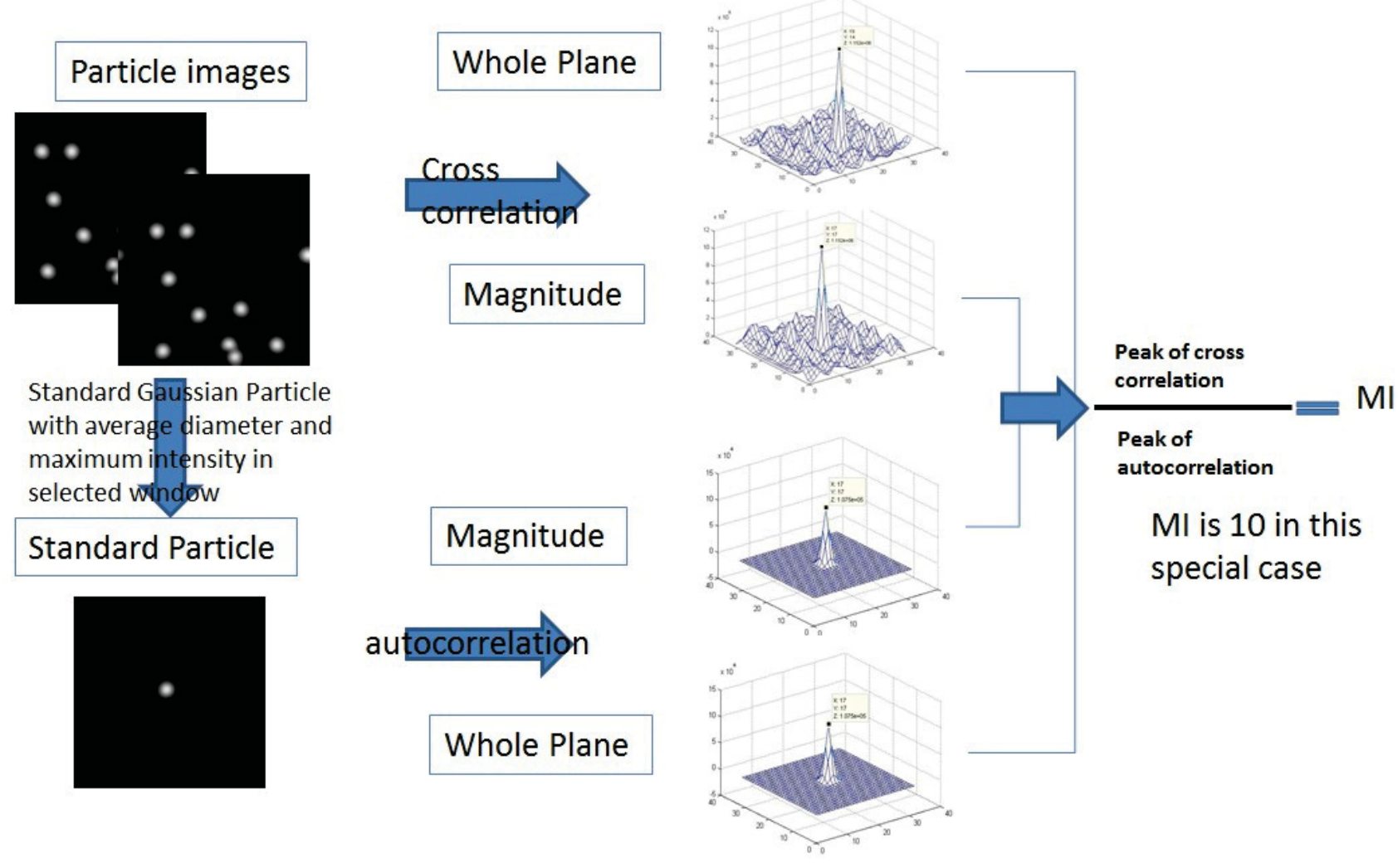

Figure 9: Schematic illustrating the calculation of MI.

The image of one representative particle can be written as:

$$
P(X, Y)=\iint J_{0} e^{\left(-8\left(\frac{\left(X-X^{\prime}\right)^{2}}{d_{0}^{2}}+\frac{\left(Y-Y^{\prime}\right)^{2}}{d_{0}^{2}}\right)\right)} \otimes \delta\left(X^{\prime}-x_{p}, Y^{\prime}-y_{p}\right) d X^{\prime} d Y^{\prime}
$$

where $\mathrm{J}_{0}$ and $\mathrm{d}_{0}$ are the intensity and diameter of the particle, $\mathrm{x}_{\mathrm{p}}$ and $\mathrm{y}_{\mathrm{p}}$ are the particle center coordinates. Then the autocorrelation can be calculated as:

$$
A(s, t)=\iint P(X, Y) \times P(X+s, Y+t) d X d Y
$$


The autocorrelation peak height is the magnitude of autocorrelation plane:

$A_{0}=\frac{1}{16} J_{0}^{2} d_{0}^{2} \pi$

We can write the analytical form of the cross correlation plane for Image 1 and Image 2 as:

$R(s, t)=\iint I_{1}(X, Y) I_{2}(X+s, Y+t) d X d Y$

$I_{1}$ and $I_{2}$ are the expressions for Image 1 and 2, with removing the background image noise effect we can consider the image as the summation of all particles within the window area:

$$
\begin{aligned}
& I_{1}(X, Y)=\iint \sum_{p 1} J_{i 1} e^{\left(-8\left(\frac{\left(X-X^{\prime}\right)^{2}}{d_{n}{ }^{2}}, \frac{\left(Y-Y^{\prime}\right)^{2}}{d_{n}{ }^{2}}\right)\right.} \otimes \delta\left(X^{\prime}-x_{p}, Y^{\prime}-y_{p}\right) d X^{\prime} d Y^{\prime} \\
& I_{2}(X, Y)=\iint \sum_{p 2} J_{i 2} e^{\left(-8\left(\frac{\left(X-X^{\prime}\right)^{2}}{d_{12}^{2}}, \frac{\left(Y-Y^{\prime}\right)^{2}}{d_{12}^{2}}\right)\right.} \otimes \delta\left(X^{\prime}-\left(x_{p}+\Delta x\right), Y^{\prime}-\left(y_{p}+\Delta y\right)\right) d X^{\prime} d Y^{\prime}
\end{aligned}
$$

where $\mathrm{p}_{1}$ and $\mathrm{p}_{2}$ are the number of particles in Image 1 and $2, \mathrm{~J}_{\mathrm{i}}$ and $\mathrm{d}_{\mathrm{i}}$ are the intensity and diameter of the $\mathrm{i}^{\text {th }}$ particle.

As mentioned before, the cross correlation peak can be considered as the summation of the autocorrelation of all correlated particles in both. By assuming the correlated particles in Frame 1 and Frame 2 are identical $\left(\mathrm{J}_{\mathrm{i} 1}=\mathrm{J}_{\mathrm{i} 2}, \mathrm{~d}_{\mathrm{i} 1}=\mathrm{d}_{\mathrm{i} 2}\right)$, we can show that the primary peak height of the cross correlation plane is:

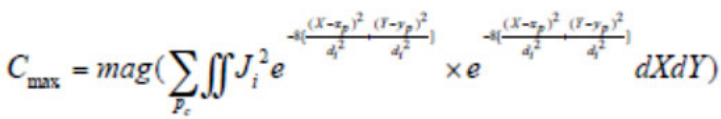

$$
\begin{aligned}
& =\sum_{p_{c}} \frac{1}{16} J_{i}^{2} d_{i}^{2} \pi
\end{aligned}
$$

where $p_{c}$ is the number of correlated particles in both frames, $J_{i}$ and $d_{i}$ are the intensity and diameter of the $\mathrm{i}^{\text {th }}$ particle. Thus the number of correlated particle pairs or the amount of mutual information between consecutive frames (MI) can be estimated as:

$$
M I=\frac{C_{\max }}{A_{0}}=\frac{\sum_{p_{c}} \frac{1}{16} J_{i}^{2} d_{i}^{2} \pi}{\frac{1}{16} J_{0}^{2} d_{0}^{2} \pi}=\sum_{P_{c}} \frac{\frac{1}{16} J_{i}^{2} d_{i}^{2} \pi}{\frac{1}{16} J_{0}^{2} d_{0}^{2} \pi}
$$

It is clear then that in the case where every correlated particle has the same intensity and diameter as the reference particle $\left(\mathrm{J}_{\mathrm{i}}=\mathrm{J}_{0}, \mathrm{~d}_{\mathrm{i}}=\mathrm{d}_{0}\right)$, then MI will be equal to $\mathrm{p}_{\mathrm{c}}$. For interrogation regions where the particle size and brightness varies, the contribution of each particle will be proportionally weighted in terms of their effect on the final correlation signal.

\subsubsection{Correlation Width and Valid Measurements}

The primary peak diameter can be calculated by (Adrian and Westerweel 2011):

$$
d_{D}=\sqrt{2 d_{\tau}^{2}+\frac{4}{3} a^{2}}
$$


where $\mathrm{d}_{\tau}$ is the particle image diameter and a is a gradient parameter. But for given a correlation plane, the correlation peak width is usually calculated by performing a three-point Gaussian fit and then computing the diameter as 4 times the standard deviation for that Gaussian distribution. The location of the maximum value of that Gaussian distribution provides the sub-pixel displacement estimation for the PIV measurement. This is subject to the assumption that the true displacement is within the primary peak region. Thus, if the difference between the measured displacement and true displacement (error) is less than half of the peak diameter, the measurement should be considered as valid because the peak corresponds to the true displacement. However, previous works often use a fixed threshold value for detecting the failed measurement or outliers. Outliers are identified if the difference between the measured value and true value is larger than a pre-determined value, for example 0.5 or 1 pixel, regardless if the correct peak is detected or not. By using this criterion, the conventional definition of outliers is inconsistent with the notions of error and uncertainty. Namely, a wide peak at a location corresponding to the true displacement although it could yield errors in excess of 1 pixel, it would still be accurate but it will not be precise. Hence, using the traditional definition would inhibit the development of models for uncertainty quantification. Instead, we suggest that the criterion for a valid measurement should be based on the diameter of the correlation peak. If the error is less than half of the peak diameter, we conclude that the measurement successfully found the correct peak and it is indeed a valid measurement. Only those measurements providing the wrong primary peak are considered as invalid. An example of this "half peak diameter" criterion is shown in Figure 10. Note that the concepts of valid measurements versus outliers are different and distinct. An outlier is determined by statistical comparison with its neighbourhood while a valid or invalid measurement should be based on an independent assessment of the measurement's success or failure, regardless of the statistical properties of the neighbourhood in which it is located. Using this model, a peak that has a width wider than a single pixel due to contributions of the particle size and a large shear gradient may be correctly identifying the velocity distribution within the interrogation region even if the highest point within that peak is located more than one pixel away from the velocity value at a location in the center of the correlated image. Thus, it should not be counted as a failure, but should be instead included as a valid measurement but with a larger than normal uncertainty.

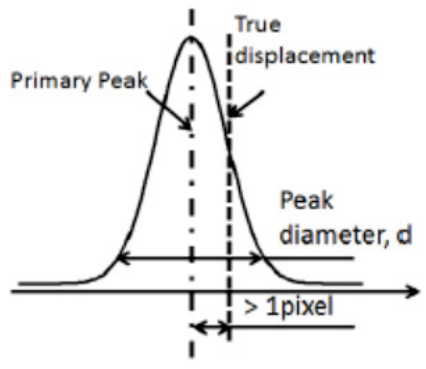

(a)

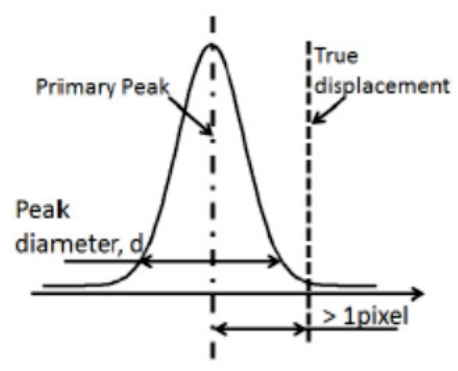

(b)

Figure 10: 1-D example of half peak diameter criterion (a) good measurement; (b) outlier. 


\subsubsection{Synthetic Image Sets}

Synthetic image sets with known displacements information were used to develop the relations between the uncertainty or error distribution and the measured metrics' value.

\section{Taylor vortex}

The first data set is 100 image pairs of an ideal Taylor vortex flow field as was previously used by Charonko and Vlachos (Charonko and Vlachos). The vortex is located at the center of the image. The maximum circumferential velocity is $\mathrm{u}_{\max }=4$ pixels/frame at distance of $\mathrm{R}_{0}=128$ pixels from the center. The velocity profile is given by:

$$
u_{\theta}(r)=u_{\max } \frac{r}{R_{0}} \exp \left[\frac{1}{2}\left(1-\frac{r^{2}}{R_{0}^{2}}\right)\right]
$$

The image size is $1024 \times 1024$ pixels. Particles in the images are Gaussian with 3-pixel diameter at the 4 standard deviation level and had 8-bit intensity resolution. Seeding density is 20 particles per $32 \times 2$ pixel window on average.

\section{Turbulent boundary layer}

The second data set is 100 image pairs of turbulent boundary layer flow field (Case B of the Second International PIV challenge in 2003 [Stanislas, Okamoto et al. 2005]). The image quality is: 70 particles pairs per $32 \times 32$ region with 2.6 pixel average particle diameter at the 4 standard deviations.

\section{Laminar separation bubble}

The last data set is 18 image pairs of laminar separation bubble flow field (Case B of the Third International PIV Challenge in 2005 [Stanislas, Okamoto et al. 2008]). 25 particles per $32 \times 32$ window is the average seeding density of this data set. The average particle diameter is about 2.0 pixels.

\subsubsection{Statistical Analysis and Uncertainty Estimation}

After we got the value of metrics mentioned before and the error of all the vectors in the three synthetic image data sets, we divided all the data points into 40 bins based on the value of the calculated metrics. Previous work has shown that the difference between the absolute magnitudes of mean velocity error and absolute mean error plus the standard deviation was very small (Eckstein and Vlachos 2009), justifying the continued use of the definition of from that work for the error distribution calculation. In each bin, the $r m s \delta_{v}$ is calculated as:

$$
n m s \hat{\delta}_{v, i}=\sqrt{\left(\operatorname{mean}\left(\hat{\delta}_{v, i}{ }^{2}\right)\right)}=\sqrt{\frac{1}{N} \sum_{i=1}^{N} \hat{\delta}_{v, i}{ }^{2}}
$$

where $\mathrm{N}$ is the number of data points in the $\mathrm{i}^{\text {th }}$ bin.

Measurement errors were first estimated at the standard uncertainty level, which should reflect one standard deviation level for the parent population of possible errors from which the true error on some measurement of the metric is drawn without respect of the exact shape of that distribution. The expanded uncertainty was calculated by making the assumption that all errors were drawn from normal distribution and the large sample assumption applied. We multiplied the standard uncertainty by a coverage factor, $\mathrm{t}_{\mathrm{CI}}$ to get the expanded uncertainty. In this work we took $\mathrm{t}_{95}=2.0$, which means the true value of the measured quantity lies within a range bounded by the measured value plus or minus the expanded uncertainty (twice the standard uncertainty) $95 \%$ of the time. If the uncertainty model is correct, $95 \%$ of all data points will have a velocity error within the provided uncertainty range. 


\subsubsection{Experimental Validation}

\subsubsection{Result and Discussion}

\section{Mutual information (MI)}

As mentioned above, the $\mathrm{MI}$ is a more general form of $\mathrm{N}_{\mathrm{I}} \mathrm{F}_{\mathrm{I}} \mathrm{F}_{\mathrm{O}}$ combining the effects of particle intensity and diameter. To further prove the above idea, PIV standard image sets (Okamoto, Nishio et al. 2000) were tested to show the relationship between MI and $\mathrm{N}_{I} \mathrm{~F}_{I} \mathrm{~F}_{\mathrm{O}}$. This data set was selected because in addition to the true velocity, the position and diameter of each particle in the image were also reported, which was necessary for a comparison between the two metrics. As mentioned before, different particles contribute differently in building the correlation plane, and thus the number of correlated particles $\left(\mathrm{N}_{\mathrm{I}} \mathrm{F}_{\mathrm{I}} \mathrm{F}_{\mathrm{O}}\right)$ must be scaled by the particle intensity and diameter within each window. The result is shown in Figure 11. The X-axis shows the value of MI and the Y-axis shows the value of intensity scaled $\mathrm{N}_{\mathrm{I}} \mathrm{F}_{\mathrm{I}} \mathrm{F}_{\mathrm{O}}$. It is clear that most of the results are aligned along the white dash line corresponding to $\mathrm{MI}=\mathrm{N}_{\mathrm{I}} \mathrm{F}_{\mathrm{I}} \mathrm{F}_{\mathrm{O}}$ supporting the notion that the MI provides a direct estimate of the apparent $\mathrm{N}_{\mathrm{I}} \mathrm{F}_{\mathrm{I}} \mathrm{F}_{\mathrm{O}}$ for an individual image pair.

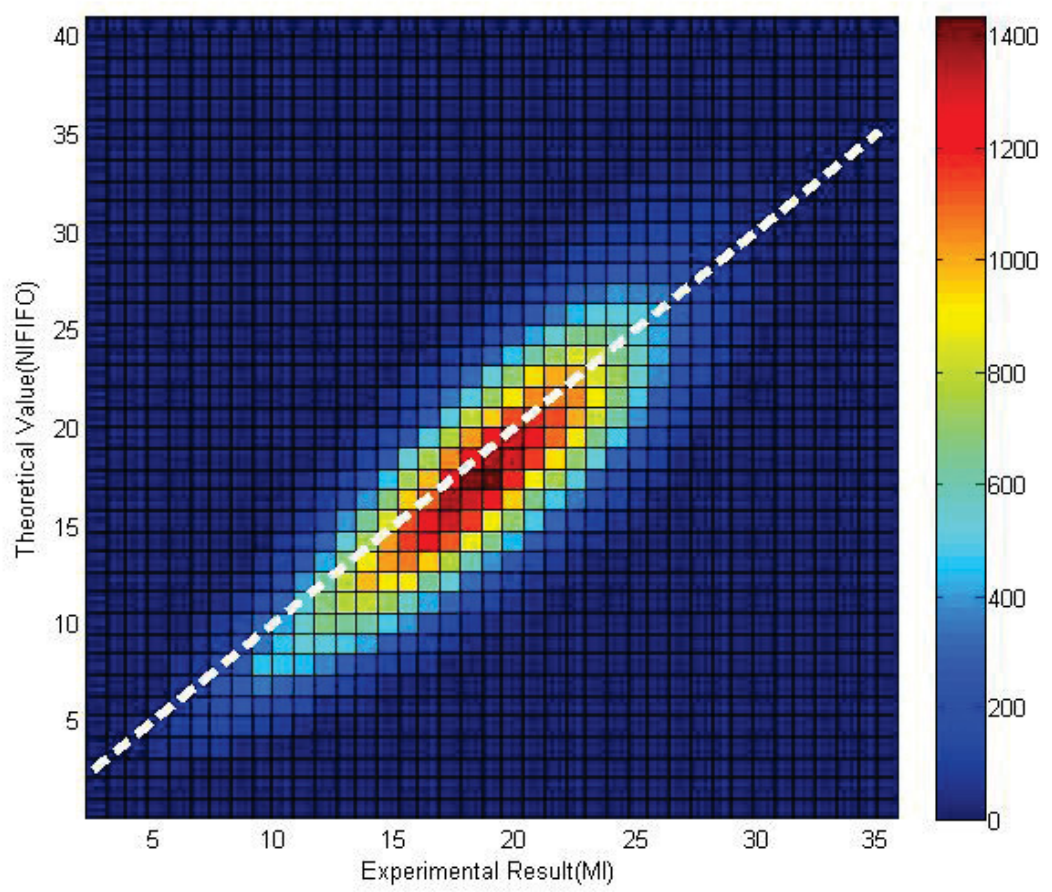

Figure 11: Mutual Information (MI) $\mathrm{VS} \mathrm{N}_{\mathrm{I}} \mathrm{F}_{\mathrm{I}} \mathrm{F}_{\mathrm{O}}$. Color represents number of measurements in each bin.

The relationship between the mutual information and the distribution of velocity error for Case B of 2003 PIV Challenge using standard cross correlation method with $32 \times 32$ window size is shown in Figure 12. Details of statistical analysis and error distribution calculation are provided in (Charonko and Vlachos). The scatter plot shows all the combinations of MI and error. It is obvious that large errors are expected when the MI value is small. As shown in the plot, almost no measurement had both large error and high MI value in this special case. Moreover, MI shows a 95\% valid detection probability for MI values larger than 5 , which is consistent with previous findings for the valid vector detection probability versus $\mathrm{N}_{\mathrm{I}} \mathrm{F}_{\mathrm{I}} \mathrm{F}_{\mathrm{O}}$ (Keane and Adrian 1992). When the MI value is below 5, the probability drops rapidly. This result further supports that $\mathrm{MI}$ and $\mathrm{N}_{\mathrm{I}} \mathrm{F}_{\mathrm{I}} \mathrm{F}_{\mathrm{O}}$ are measuring the same properties of the PIV experiment. 


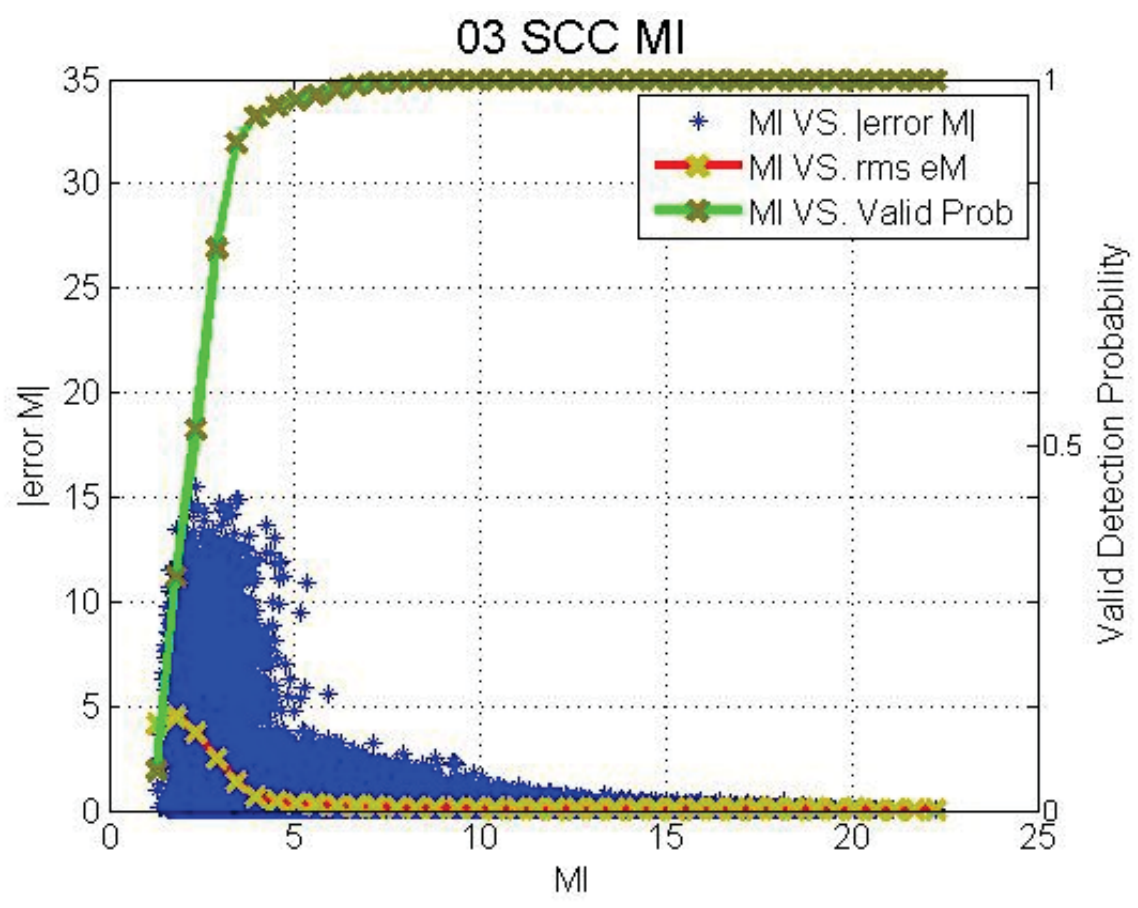

Figure 12: Plot of the distribution of velocity error using SCC processing versus correlation peak ratio for the turbulent boundary layer images of the 2003 PIV Challenge. (blue scatter dot) the measured distribution of MI value and error of velocity magnitude; (red line) mean error of velocity magnitude on each MI value; (green line) valid detection probability on each MI value.

\subsubsection{Relationship of Uncertainty Versus Cross-Correlation SNR Metrics}

\section{Uncertainty Estimation Model}

The uncertainty model which provides a relationship between any of the SNR metrics to the standard uncertainty is based on the fitting function proposed in (Eckstein and Vlachos 2009). Hence, the estimated standard uncertainty is calculated by determining the fitting parameters of the following equation:

$u^{2}=\left(M \exp \left(-\frac{1}{2}\left(\frac{\phi-N}{s}\right)^{2}\right)\right)^{2}+\left(A \phi^{B}\right)^{2}+(C)^{2}$

The first term is a Gaussian function used to account for the uncertainty due to invalid measurements which contribute uncertainty $\mathrm{M}$, where the exact value of $\mathrm{M}$ is related to the range of possible velocity measurements and the distribution of the true velocity within the sampled flow field (Charonko and Vlachos). The $(\varphi-\mathrm{N})$ term allows the error to climb rapidly as the metric's value approaches some small number, and $\mathrm{N}$ is the theoretical minimum value of the calculated metric.

Based on the definition of each quantity, we can determine analytically what value of $\mathrm{N}$ we should use for each. For PPR, the minimum value is 1 when we have a primary peak and secondary peak with the same height. Based on the definition of PRMSR, when all points in $\mathrm{C}_{\mathrm{rms}}$ have a value of half the main peak height the theoretical minimum value for PRMSR is 4 . The extreme case for PCE occurs when the peak is only slightly higher than the rest of the correlation plane, and the rest of the plane shares the same correlation value; in this case the PCE value is close to 1. Because entropy behaves the opposite way as other basic SNR metrics, we take the inverse of entropy (i.e., $\varphi=1 /$ entropy to keep the fitting function type consistent among all metrics). The theoretical minimum value for 
inverse entropy should be 0 . The theoretical minimum value for MI is also 0 when no particles correlated between two consecutive image frames.

The second term in equation 14 is the contribution to the uncertainty by the valid vectors, which means the largest uncertainty that could be expected would be governed primarily by $\mathrm{A}$ if it can be assured that the given measurement is valid. The last term $\mathrm{C}$ is a constant, which corresponds to the lowest uncertainty we can achieve. The estimated uncertainty for a measurement with a given calculated metric value is governed by the combination of the above three terms.

Although outliers were detected by using the new half peak diameter rule described earlier, it is not appropriate to develop a model for uncertainty estimation using only the valid measurements. Unlike synthetic image sets, in real experiments the true velocity field is unknown and it is inevitable that velocity fields would be contaminated by invalid measurements. Therefore both invalid and valid vectors are included in developing the uncertainty model estimation. All three synthetic cases with 3 different window size (or effective window size for RPC method), $16 \times 16,32 \times 32$, and $64 \times 64$ were included in the test providing a sample containing 12 million data points.

\subsubsection{Results of Uncertainty Estimation}

In order to keep the calculation process consistent, we applied the minimum correlation value subtraction method as described earlier to both SCC and RPC. However, this method has a minimal effect on the RPC models since phase filtering is effectively immune to background noise effects. Figure 13 shows the curve fitting results for estimating the uncertainty using peak ratio with both RPC and SCC methods after the minimum subtraction. The corresponding curve fitting functions are:

In the previously reported results for SCC processing, the fitted curve only partially agreed with the original data (Eckstein and Vlachos 2009). The current results shown in Figure 13a, show that the model fit provides agreement with the original data almost across the whole range, with $\mathrm{R}$ square value of 0.98. The model for estimating uncertainty for SCC processing provides larger values by comparison to the RPC processing, but the relationships are now much more similar than they were in the previous work that did not use minimum subtraction. The green curves show the error distribution of velocity magnitude versus PPR of good vectors only. 


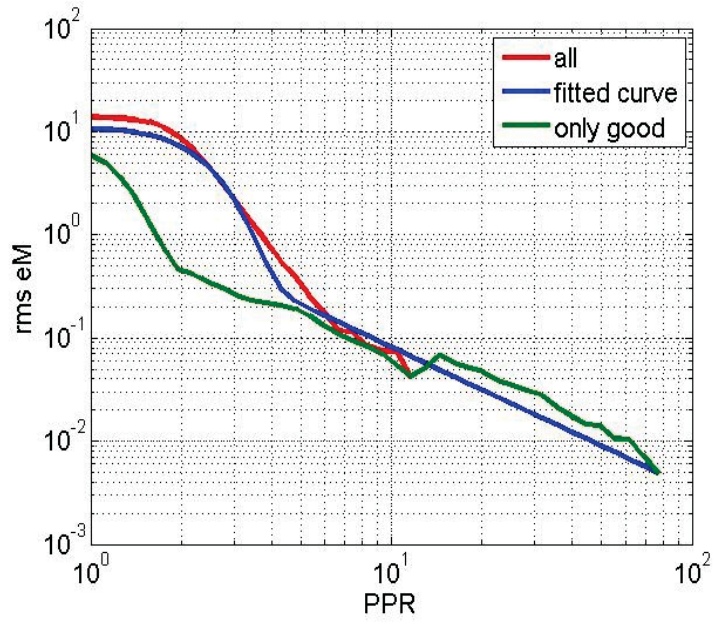

(a)

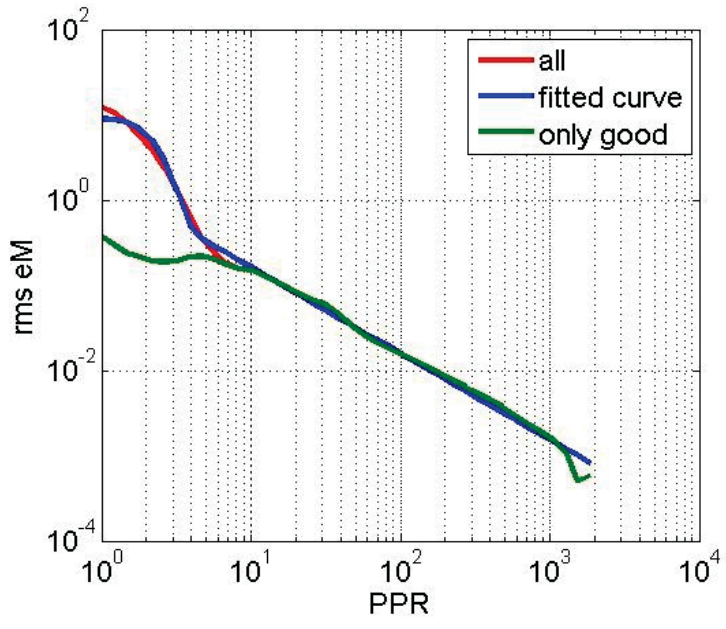

(b)

Figure 13: Plots of the relationship of the calculated standard uncertainty on velocity magnitude versus peak ratio for both the (a) SCC, and (b) RPC, for all three synthetic image sets. (red line) original curve of uncertainty on velocity magnitude versus peak ratio; (blue line) three term function fitted curve; (green line) uncertainty on velocity magnitude versus peak ratio for only valid vectors.

Figure 14 through Figure 16 show the curve fitting results for estimating the uncertainty using other basic SNR metrics with both RPC and SCC methods. The fitting functions are:

$$
\begin{aligned}
& u_{s o c}{ }^{2}=\left(\left(14.33 \exp \left(-\frac{1}{2}\left(\frac{P R M S R-4}{40.58}\right)^{2}\right)\right)^{2}+\left(58.46 P R M S R^{-0.9939}\right)^{2}+2.22 e-14^{2}\right. \\
& u_{r p c}{ }^{2}=\left(\left(9.88 \exp \left(-\frac{1}{2}\left(\frac{P R M S R-4}{99.19}\right)^{2}\right)\right)^{2}+\left(40 P R M S R^{-0.7462}\right)^{2}+2.221 e-14^{2}\right. \\
& u_{s c c^{2}}{ }^{2}=\left(\left(15.36 \exp \left(-\frac{1}{2}\left(\frac{P C E-1}{27.71}\right)^{2}\right)\right)^{2}+\left(23.27 P C E^{-0.8761}\right)^{2}+2.221 e-14^{2}\right. \\
& u_{r p c}{ }^{2}=\left(\left(11.61 \exp \left(-\frac{1}{2}\left(\frac{P C E-1}{61.06}\right)^{2}\right)\right)^{2}+\left(21.77 P C E^{-0.7353}\right)^{2}+2.221 e-14^{2}\right. \\
& u_{s c c^{2}}{ }^{2}=\left(\left(80.64 \exp \left(-\frac{1}{2}\left(\frac{\text { Entropy }}{0.4469}\right)^{2}\right)\right)^{2}+\left(1.022(\text { Entropy })^{-1}\right)^{-2.232}\right)^{2}+0.0122^{2} \\
& u_{r p c}{ }^{2}=\left(\left(26.19 \exp \left(-\frac{1}{2}\left(\frac{\text { Entropy }}{0.1054}\right)^{2}\right)\right)^{2}+\left(0.455\left(\text { Entropy }^{-1}\right)^{-0.4977}\right)^{2}+2.241 e-14^{2}\right.
\end{aligned}
$$

All these functions agree that the uncertainty would be larger for the SCC than the RPC for the same value of each metric. It is interesting to note that the maximum uncertainty predicted for PRMSR and PCE can be as large as 20 pixels even for valid measurements for both SCC and RPC methods. Despite the theoretical minimum value for PRMSR and PCE, in fact the PRMSR and PCE value is typically hundred times higher than the theoretical minimum value, so the uncertainty for valid vectors would never be that high for real cases. All these functions showed an acceptable agreement with the raw data, and the corresponding $\mathrm{R}^{2}$ values for each function are shown in Table 2. 
Table 2: $\mathrm{R}^{2}$ vale of all fitting functions.

$\begin{array}{ccccc} & \text { PPR } & \text { PRMSR } & \text { PCE } & \text { Entropy } \\ \text { SCC } & 0.98 & 0.99 & 0.99 & 0.98 \\ & & & & \\ \text { RPC } & 0.99 & 0.97 & 0.95 & 0.92\end{array}$

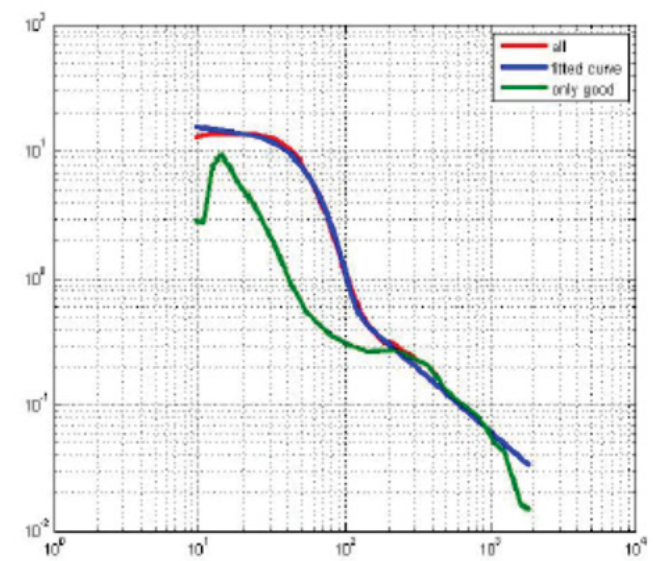

(a)

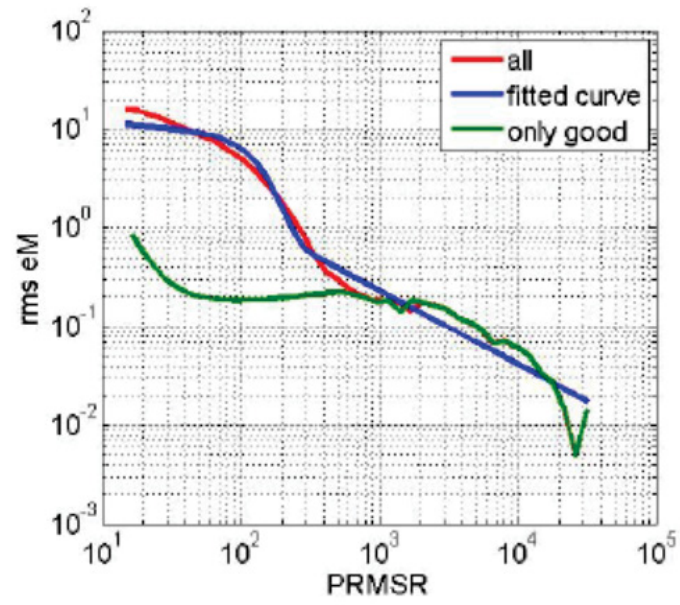

(b)

Figure 14: Plots of the relationship of the calculated standard uncertainty on velocity magnitude versus PRMSR for both the (a) SCC, and (b) RPC, for all three synthetic image sets. (red line) original curve of uncertainty on velocity magnitude versus PRMSR; (blue line) three term function fitted curve; (green line) uncertainty on velocity magnitude versus PRMSR for only valid vectors.

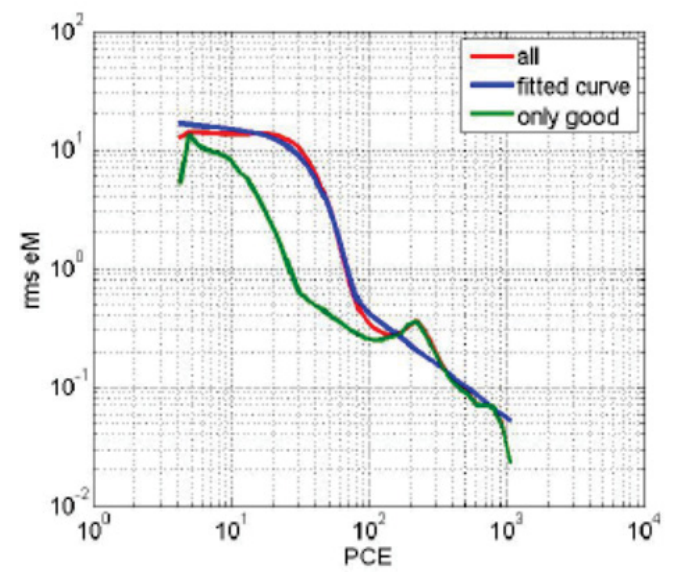

(a)

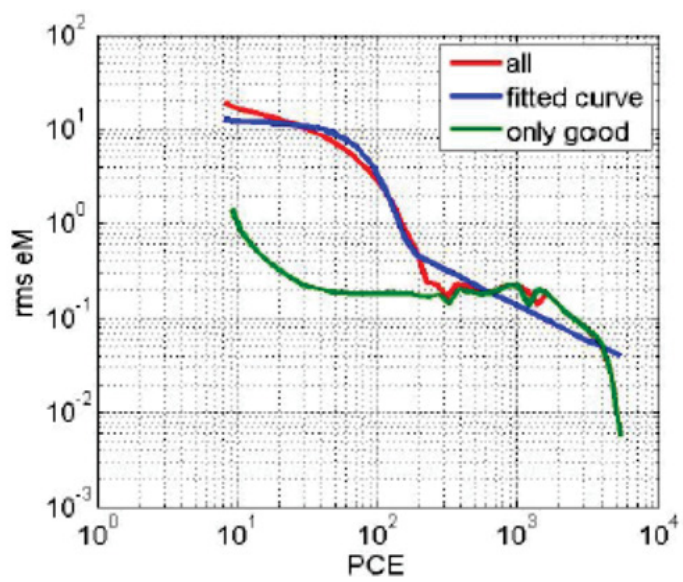

(b)

Figure 15: Plots of the relationship of the calculated standard uncertainty on velocity magnitude versus PCE for both the SCC, and (b) RPC, for all three synthetic image sets. (red line) original curve of uncertainty on velocity magnitude versus PCE; (blue line) three term function fitted curve; (green line) uncertainty on velocity magnitude versus PCE for only valid vectors. 


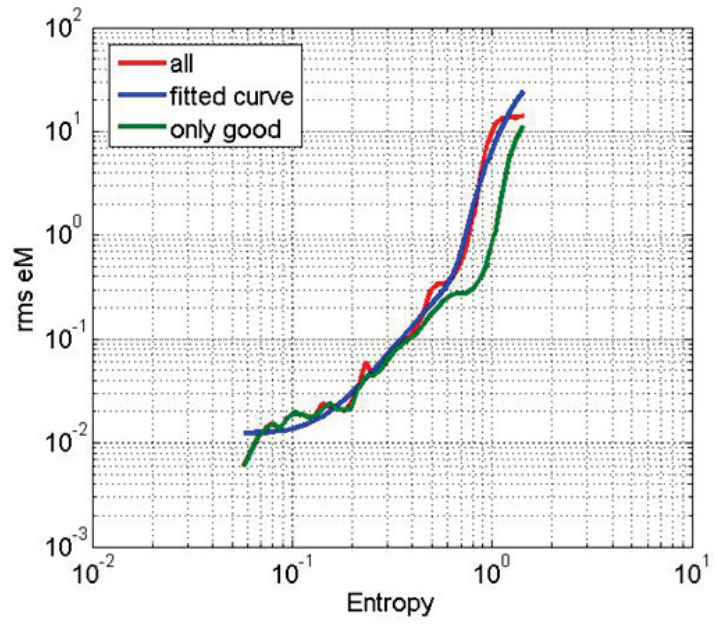

(a)

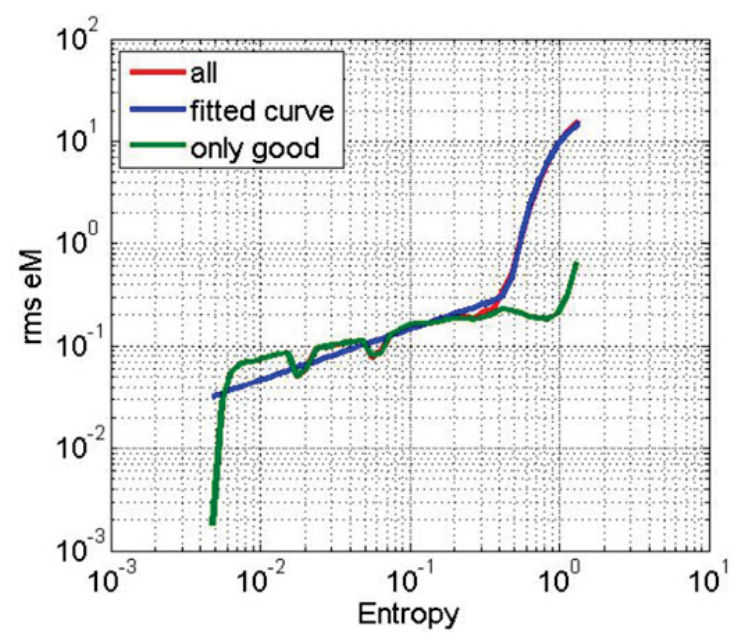

(b)

Figure 16: Plots of the relationship of the calculated standard uncertainty on velocity magnitude versus Entropy for both the (a) SCC, and (b) RPC, for all three synthetic image sets. (red line) original curve of uncertainty on velocity magnitude versus Entropy; (blue line) three term function fitted curve; (green line) uncertainty on velocity magnitude versus Entropy for only valid vectors.

The curve fitting for MI was done using all three synthetic data sets with only $32 \times 32$ window size, as shown in Figure 10. The fitting functions are:

$$
\begin{aligned}
& u_{s c c^{2}}{ }^{2}=\left(\left(26.22 \exp \left(-\frac{1}{2}\left(\frac{M I}{2.144}\right)^{2}\right)\right)^{2}+\left(0.8739 M M^{-0.9439}\right)^{2}+0.05^{2}\right. \\
& u_{p p c}{ }^{2}=\left(\left(2.382 \exp \left(-\frac{1}{2}\left(\frac{M I}{2.056}\right)^{2}\right)\right)^{2}+\left(0.3401 M M^{-0.7386}\right)^{2}+(2.23 e-14)^{2}\right.
\end{aligned}
$$

The $\mathrm{R}^{2}$ value is 0.99 for SCC model, and 0.98 for RPC model. The green curve shows the rms error of velocity magnitude only for valid vectors, which is almost linear in the logarithm domain. This fact further supports our assumption that the power-law term counts for uncertainty of valid measurements. It is interesting to note that the constant term in the SCC model is 0.05 pixels, which matches well with the widely accepted value about the expected accuracy of PIV measurements under ideal (simulated) conditions (Huang, Dabiri et al. 1997). The RPC function is similar to SCC with smaller error value. The curve for the RPC is not as smooth as the SCC one because in RPC processing the magnitude part of correlation which contains the particle image information is removed. As a result the MI value captures only the contribution of the loss of correlation and not the particle number density. This is a limitation of the current formulation that will be addressed in the future.

The above equations are used for estimating the standard uncertainty using the corresponding metrics. This standard uncertainty was then mutiplied by a coverage factor of $t_{95}=2.0$ to yield an estimate of the uncertainty at the $95 \%$ confidence interval using the large sample approximation for a normal error distribution. Finally, the percent coverage of the expanded uncertainty was calculated in comparison to the exact true error for each velocity measurement according to the following formula:

$$
\text { coverage }=\frac{\# \text { of estimates for which } \delta_{V} \leq \mathrm{U}_{V}}{\text { total } \# \text { of velocity estimates }} \times 100 \%
$$

The coverage should be close to $95 \%$ for the expanded uncertainty if the uncertainty estimation was correct on average. The exact values of coverage factor of all functions using all three synthetic data 
sets with $16 \times 16,32 \times 32$ and $64 \times 64$ window sizes (for MI, we use $32 \times 32$ window size only) are listed in Table 3 . All of the coverage factors are very close to $95 \%$.

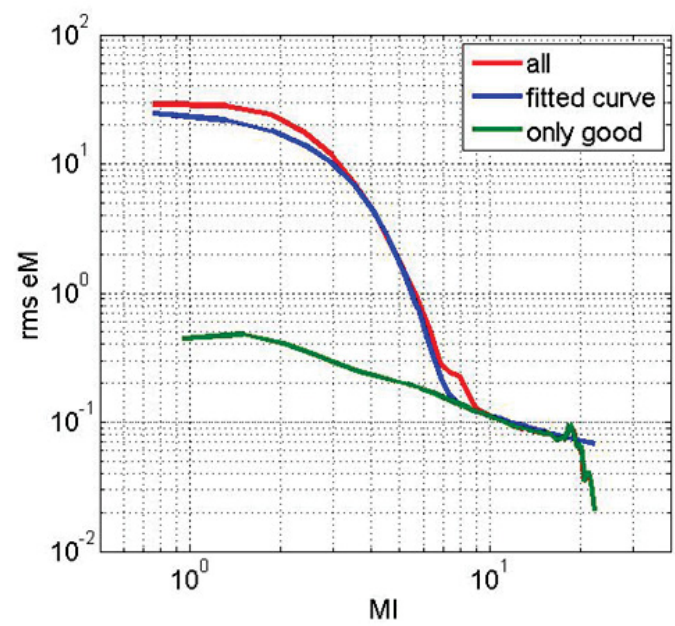

(a)

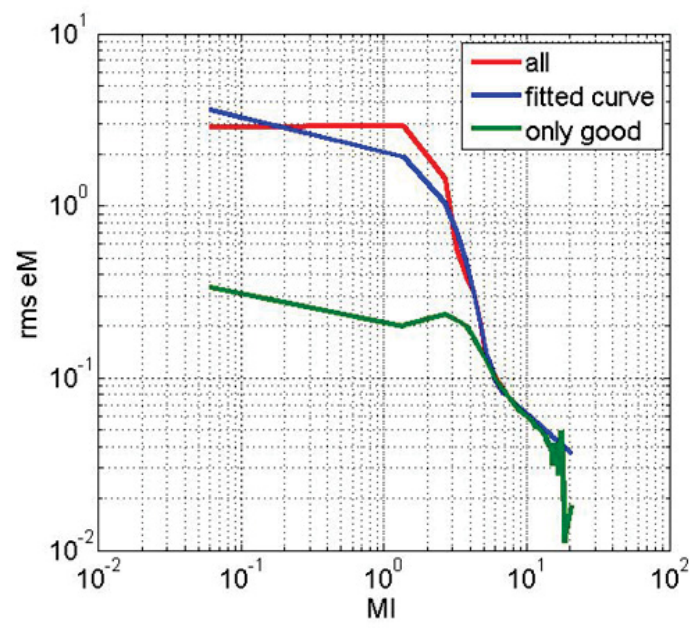

(b)

Figure 17:Plots of the relationship of the calculated standard uncertainty on velocity magnitude versus MI for both the (a) SCC, and (b) RPC, for all three synthetic image sets. (red line) original curve of uncertainty on velocity magnitude versus MI; (blue line) three term function fitted curve; (green line) uncertainty on velocity magnitude versus MI for only valid vectors.

Table 3: Coverage vale of all fitting functions with synthetic data sets

$\begin{array}{cccccc} & \text { PPR } & \text { PRMSR } & \text { PCE } & \text { Entropy } & \text { MI } \\ \text { SCC } & 95.9 \% & 95.6 \% & 96.1 \% & 95.4 \% & 95.7 \% \\ \text { RPC } & 94.9 \% & 94.3 \% & 94.7 \% & 94.7 \% & 96.0 \%\end{array}$

\subsubsection{Application to Real Flow Fields}

Our uncertainty models were further tested with real experimental data. In this work, we are using the same data set of stagnation plate flow used by Charonko and Vlachos (Charonko and Vlachos). The experimental details can be found therein. The details of calculating the time average field and then the uncertainty introduced by the fitting process were also described in (Charonko and Vlachos).

Afterwards the combined standard uncertainty from both PIV correlation and the experimental fit for the "true" field is multiplied by a factor of 2 to yield the expanded uncertainty $U_{\text {total, }}$ and finally the coverage factor is calculated by the following formula:

coverage $=\frac{\# \text { of estimates for which } \delta_{\mathrm{v}} \leq \mathrm{U}_{\text {total }}}{\text { total } \# \text { of velocity estimates }} \times 100 \%$

The exact values of coverage factor of each function using the real experiment data set with $32 \times 32$ window sizes are listed in Table 3 . Values of all coverage factors are close to $95 \%$. 
Table 4: Coverage vale of all fitting functions with real experiment sets.

$\begin{array}{cccccc} & \text { PPR } & \text { PRMSR } & \text { PCE } & \text { Entropy } & \text { MI } \\ \text { SCC } & 95.2 \% & 94.0 \% & 96.1 \% & 94.1 \% & 95.4 \% \\ \text { RPC } & 95.3 \% & 95.1 \% & 96.4 \% & 94.7 \% & 96.4 \%\end{array}$

\subsubsection{Important Findings}

In this report, we show that cross-correlation SNR metrics calculated exclusively from the correlation plane can be used to estimate the uncertainty of the PIV measurements. In the first part of our work, metrics of basic correlation SNR related to the peak detectability are introduced. We also develop a new metric termed Mutual Information (MI) to estimate the real and apparent $\mathrm{N}_{\mathrm{I}} \mathrm{F}_{\mathrm{I}} \mathrm{F}_{\mathrm{O}}$ directly from the calculated correlation plane. Both theoretical derivation and experimental results support that MI corresponds to the apparent $\mathrm{N}_{\mathrm{I}} \mathrm{F}_{\mathrm{F}} \mathrm{F}_{\mathrm{O}}$ and would be a practical measure of the correlation SNR with direct connection to the established PIV theory. A simple but consequential correction on the correlation plane is introduced using a minimum correlation value subtraction to remove the effect of the background image noise and thus improve the model's performance for uncertainty estimation.

The relationship between the uncertainty and the metrics of correlation SNR of individual velocity measurements were explored using both robust phase correlation (RPC) and standard cross correlation (SCC) method. The standard uncertainty is governed by a well-defined relationship between the correlation SNR using both methods. This relation is quantified using a three-term formulation for both processing methods. In the three-term function, the Gaussian distribution term is related to probability of occurrence of invalid measurements; the power-law term describes the primary behavior of the uncertainty versus the metrics; and a constant expresses the minimum expected uncertainty level for the corresponding methodology, regardless of value of the metrics. The formulas successfully predicted the expanded uncertainty coverage close to $95 \%$ over all three synthetic image sets as well as a $2 \mathrm{D}$ stagnation point real experiment case using all provided metrics using both SCC and RPC method.

In conclusion, this paper provides a general framework of models for predicting the expected uncertainty levels for individual velocity measurement in a PIV flow field without the knowledge of local flow conditions using only the information contained in the calculated correlation plane. This work continues work establishing the foundations towards the growing understanding of PIV uncertainty estimation.

\subsubsection{Current Status and Future Work}

Ongoing work is focusing on the following three objectives: (1) expand signal to noise ratio method to provide uncertainty upper and lower bounds (2) develop a direct estimation of the uncertainty from the correlation surface and eliminate the need for an empirical model, and (3) expand the framework to stereoscopic planar (2D-3C) PIV data.

\subsection{Propagation of Uncertainty to Statistics}

Once uncertainties are in hand for each component of velocity at each location at each instant, these must be propagated into more useful quantities for CFD validation, such as the mean velocity and Reynolds Stresses. The Data Reduction Equations (DREs) for doing so are simply the definitions of the quantities of interest. One can propagate using either a Taylor Series Method (TSM) or a Monte Carlo Method (MCM) [cite Coleman and Steel]. Both methods have limitations. The Taylor Series Method 
requires one to assume that all random errors are distributed normally, and that all uncertainties are symmetric about the nominal value. Unfortunately, the distribution of a quantity such as the square of a velocity cannot be normally distributed, since all values are $>0$. In Wilson and Smith (2013) it is shown that while the uncertainty bands of mean velocities computed from PIV measurements are generally close to symmetric about the mean, the uncertainties of Reynolds stresses are generally one-sided and negative (meaning that PIV tends to overestimate Reynolds stresses). The results of this study are independent of the method used to compute the instantaneous uncertainties and are incorporated into the PIV Stats code, which is available on the EFDL website.

\subsection{Propagation of Two---Component Uncertainty to Three--- Component (Stereo)}

Since 3-component (stereo) Particle Image Velocimetry (PIV) velocity fields are derived from two, 2-compnent $(2 \mathrm{C})$ fields, random uncertainties from the $2 \mathrm{C}$ fields clearly propagate into the $3 \mathrm{C}$ field. $\mathrm{We}$ have developed a framework and tools to perform such a propagation using commercial PIV software and the USM method, although the propagation works similarly for any $2 \mathrm{C}$ random uncertainty method. Stereo calibration information is needed to perform this propagation. As a starting point, a pair of $2 \mathrm{C}$ uncertainty fields is combined in exactly the same manner as velocity fields to form a $3 \mathrm{C}$ uncertainty field using commercial software.

For a common PIV camera set up, the $x-y$ plane coincides with the laser sheet plane with the $z$ - direction is perpendicular as shown in Figure 18. The cameras view the laser sheet from angles $\alpha 1$ and $\alpha 2$ taken normal from the laser plane.

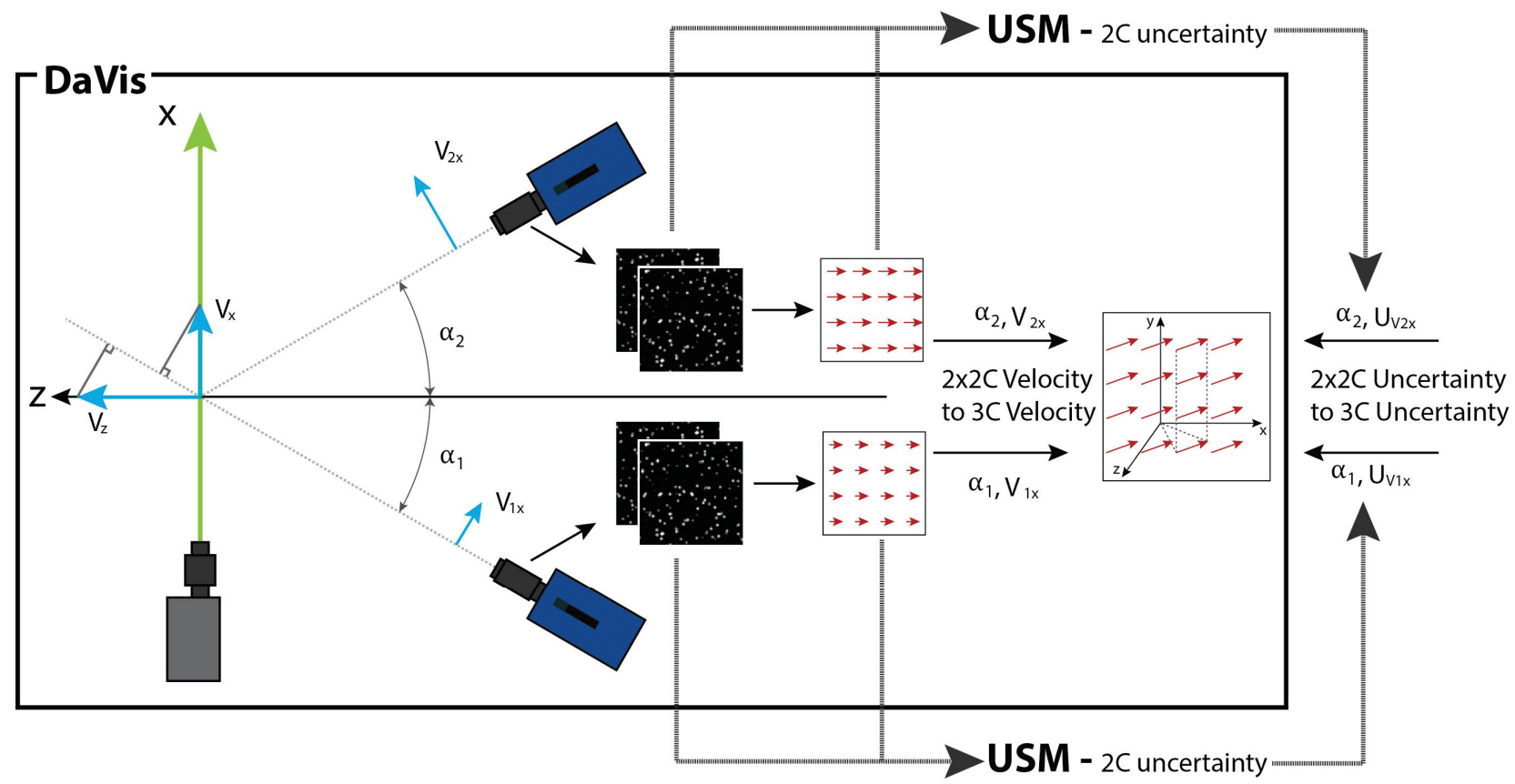

Figure 18: Uncertainty estimation using the Uncertainty Surface Method (USM).

The $x$-velocities $\left(V_{1 x}\right.$ and $\left.V_{2 x}\right)$ and the $y$-velocities $\left(V_{1 y}\right.$ and $\left.V_{2 y}\right)$ viewed by Camera 1 and 2 are given by

$$
\begin{aligned}
& V_{1 x}=V_{x} \cos \alpha_{1}+V_{z} \sin \alpha_{1} \\
& V_{2 x}=V_{x} \cos \alpha_{2}+V_{z} \sin \alpha_{2}
\end{aligned}
$$

and 
$V_{1 y}=V_{2 y}=V_{y}$

where $V_{x}, V_{y}$, and $V_{z}$ are the velocity components based on the combination of both $2 \mathrm{C}$ measurements.

Equations 27 and 28 solved for the $3 \mathrm{C}$ velocities $V_{x}$ and $V_{z}$ provide

$V_{x}=\left(V_{1 x} \sin \alpha_{2}-V_{2 x} \sin \alpha_{1}\right) / \sin \left(\alpha_{2}-\alpha_{1}\right)$

$V_{z}=\left(V_{1 x} \cos \alpha_{2}-V_{2 x} \cos \alpha_{1}\right) / \sin \left(\alpha_{2}-\alpha_{1}\right)$,

while $V_{y}$ is simply the average of the measurements from the two cameras:

$V_{y}=\left(V_{1 y}+V_{2 y}\right) / 2$.

The Taylor Series Method (TSM) for propagation of uncertainty is described in Coleman and Steele (2009). The data reduction equations are Eqs. 30-32 where $V_{x}=V\left(V_{l x}, V_{2 x}\right), V_{y}=V\left(V_{l y}, V_{2 y}\right)$, and $V_{z}=V\left(V_{l x}\right.$, $V_{2 x}$ ). The uncertainty in $V_{x}$ is given by

$$
U_{V_{x}}=\sqrt{\left(U_{V_{1 x}}\right)^{2}\left(\frac{\partial V_{x}}{\partial V_{1 x}}\right)^{2}+\left(U_{V_{2 x}}\right)^{2}\left(\frac{\partial V_{x}}{\partial V_{2 x}}\right)^{2}-2 U_{V_{1 x}} U_{V_{2 x}}\left(\frac{\partial V_{x}}{\partial V_{1 x}}\right)\left(\frac{\partial V_{x}}{\partial V_{2 x}}\right)}
$$

Inserting the partial derivatives of $V_{x}$ from Eq. 30 results in

$$
U_{V_{x}}=\sqrt{\left(U_{V_{1 x}}\right)^{2}\left(\frac{\sin \alpha_{2}}{\sin \left(\alpha_{2}-\alpha_{1}\right)}\right)^{2}+\left(U_{V_{2 x}}\right)^{2}\left(\frac{\sin \alpha_{1}}{\sin \left(\alpha_{2}-\alpha_{1}\right)}\right)^{2}-2 U_{V_{1 x}} U_{V_{2 x}} \frac{\sin \alpha_{1} \sin \alpha_{2}}{\sin \left(\alpha_{2}-\alpha_{1}\right)} .}
$$

Similarly the uncertainty in $V_{y}$ and $V_{z}$ are

$U_{V_{z}}=\sqrt{\left(U_{V_{1 x}}\right)^{2}\left(\frac{\cos \alpha_{2}}{\sin \left(\alpha_{2}-\alpha_{1}\right)}\right)^{2}+\left(U_{V_{2 x}}\right)^{2}\left(\frac{\cos \alpha_{1}}{\sin \left(\alpha_{2}-\alpha_{1}\right)}\right)^{2}-2 U_{V_{1 x}} U_{V_{2 x}} \frac{\cos \alpha_{1} \cos \alpha_{2}}{\sin \left(\alpha_{2}-\alpha_{1}\right)}}$

and

$U_{V_{y}}=\frac{1}{2} \sqrt{\left(U_{V_{1 y}}\right)^{2}+\left(U_{V_{2} y}\right)^{2}+2 U_{V_{1 y}} U_{V_{2 y}}}$

By factoring the quantities within the square roots of Eqs. 33-35, they become

$$
\begin{aligned}
& U_{V_{x}}=\sqrt{\left(\left(U_{V_{1 x}} \sin \alpha_{2}-U_{V_{2 x}} \sin \alpha_{1}\right) / \sin \left(\alpha_{2}-\alpha_{1}\right)\right)^{2}} \\
& U_{V_{z}}=\sqrt{\left(\left(U_{V_{1 x}} \cos \alpha_{2}-U_{V_{2 x}} \cos \alpha_{1}\right) / \sin \left(\alpha_{2}-\alpha_{1}\right)\right)^{2}}
\end{aligned}
$$

and

$U_{V_{y}}=\frac{1}{2} \sqrt{\left(U_{V_{1 y}}+U_{V_{2 y}}\right)^{2}}$

In this form, the square roots and squares in Eqs. 37-39 cancel, resulting in

$$
\begin{aligned}
& U_{V_{x}}=\left(U_{V_{1 x}} \sin \alpha_{2}-U_{V_{2 x}} \sin \alpha_{1}\right) / \sin \left(\alpha_{2}-\alpha_{1}\right) \\
& U_{V_{z}}=\left(U_{V_{1 x}} \cos \alpha_{2}-U_{V_{2 x}} \cos \alpha_{1}\right) / \sin \left(\alpha_{2}-\alpha_{1}\right) \\
& \quad \text { and }
\end{aligned}
$$


$U_{V_{y}}=\left(U_{V_{1 y}}+U_{V_{2 y}}\right) / 2$

Therefore, the equations used to calculate the 3C velocities (Eqs. 30-32) are equivalent to those used to calculate the 3C uncertainties (Eqs. 40-42) when the velocity uncertainty is used in place of velocity. The 2C uncertainties $\left(U v_{1 x}, U v_{l y}\right.$, and $U v_{2 y}$, $)$ can be obtained by implementing the Uncertainty Surface Method (USM), or any other $2 \mathrm{C}$ UQ method. The angles are imbedded in the stereo calibration information for a polynomial fit calibration and are an output of a pinhole calibration. In the case of the polynomial calibration, DaVis may be used to perform the calculations in Eqs. 40-42 as outlined in Appendix B-1, B-2 and B-3.

Equations 30-32 are currently used in the PIV software package DaVis by LaVision to combine the 2C velocity fields into a single $3 \mathrm{C}$ velocity field when a pinhole calibration is used. In a similar fashion, the $2 \times 2 \mathrm{C}$ uncertainty vector fields can be read into $\mathrm{DaV}$ is and combined using the same method as described in Figure 18.

\section{REFERENCES}

Adrian, R. J. (1991). "Particle-Imaging Techniques for Experimental Fluid-Mechanics." Annual Review of Fluid Mechanics 23: 261-304.

Adrian, R. J. (2005). "Twenty years of particle image velocimetry.” Experiments in Fluids 39(2): 159-169.

Adrian, R. J. and J. Westerweel (2010). Particle Image Velocimetry, Cambridge Univ Pr.

Adrian, R. J. and J. Westerweel (2011). Particle Image Velocimetry. Cambridge; New York, Cambridge University Press.

Adrian, R. J. and C. S. Yao (1985). "Pulsed Laser Technique Application to Liquid and Gaseous Flows and the Scattering Power of Seed Materials." Applied Optics 24(1): 44-52.

Coleman, H.W. and W.G. Steele (2009). Experimentation, Validation, And Uncertainty Analysis For Engineers, 3rd edn. Wiley, Hoboken, NJ.

Charonko, J. J. and P. P. Vlachos (2013). "Estimation of uncertainty bounds for individual particle image velocimetry measurements from cross-correlation peak ratio." Measurement Science and Technology 24(6): 065301.

Eckstein, A. and P. P. Vlachos (2009). "Assessment of advanced windowing techniques for digital particle image velocimetry (DPIV).” Measurement Science and Technology 20(7): 075402.

Eckstein, A. C., J. Charonko and P. Vlachos (2008). "Phase correlation processing for DPIV measurements." Experiments in Fluids 45(3): 485-500.

Grant, I. (1997). "Particle image velocimetry: A review." Proceedings of the Institution of Mechanical Engineers Part C-Journal of Mechanical Engineering Science 211(1): 55-76.

Hain, R. and C. J. Kahler (2007). "Fundamentals of multiframe particle image velocimetry (PIV)." Experiments in Fluids 42(4): 575-587.

Huang, H., D. Dabiri and M. Gharib (1997). "On errors of digital particle image velocimetry." Measurement Science and Technology 8(12): 1427.

Keane, R. and R. Adrian (1992). "Theory of cross-correlation analysis of PIV images.” Applied Scientific Research 49(3): 191-215.

Keane, R. D. and R. J. Adrian (1990a). "Optimization of Particle Image Velocimeters .1. Double Pulsed Systems.” Measurement Science \& Technology 1(11): 1202-1215.

Keane, R. D. and R. J. Adrian (1990b). "Optimization of particle image velocimeters. I. Double pulsed systems." Measurement Science and Technology 1(11): 1202. 
Keane, R. D. and R. J. Adrian (1991). "Optimization of particle image velocimeters: II. Multiple pulsed systems.” Measurement Science and Technology 2(10): 963.

Kompenhans, J., M. Raffel, S. T. Wereley and C. E. Willert (2007). Particle image velocimetry.

Kumar, B. V. K. V. and L. Hassebrook (1990). "Performance measures for correlation filters." Appl. Opt. 29(20): 2997-3006.

Nobach, H. and E. Bodenschatz (2009) "Limitations Of Accuracy In PIV Due To Individual Variations Of Particle Image Intensities.” Experiments In Fluids 47(1):27-38, DOI 10.1007/s00348-009-0627-4.

Okamoto, K., S. Nishio, T. Kobayashi, T. Saga and K. Takehara (2000). "Evaluation of the 3D- PIV Standard Images (PIV-STD Project).” Journal of Visualization 3(2): 115-123.

Persoons, T. and T. S. O’Donovan (2010). "High Dynamic Velocity Range Particle Image Velocimetry Using Multiple Pulse Separation Imaging." Sensors 11(1): 1-18.

R, M. (1983). "Mesure de champs de vitesse d' ecoulements fluides par analyse de suites d' images obtenues par diffusion d' un feuillet lumineux." Ph.D. thesis Facult'e des Sciences Appliqu' ees, Universite Libre de Bruxelles.

Scarano, F. (2002). "Iterative image deformation methods in PIV." Measurement Science \& Technology 13(1): R1-R19.

Scarano, F. (2003). "Theory of non-isotropic spatial resolution in PIV." Experiments in Fluids 35(3): 268-277.

Scarano, F. (2004). "A super-resolution particle image velocimetry interrogation approach by means of velocity second derivatives correlation." Measurement Science \& Technology 15(2): 475-486.

Sciacchitano, A., B. Wieneke and F. Scarano (2013). "PIV uncertainty quantification by image matching." Measurement Science and Technology 24(4): 045302.

Shannon, C. E. (2001). "A mathematical theory of communication." SIGMOBILE Mob. Comput. Commun. Rev. 5(1): 3-55.

Soloff, S. M., R. J. Adrian and Z. C. Liu (1997). "Distortion compensation for generalized stereoscopic particle image velocimetry." Measurement Science \& Technology 8(12): 1441- 1454.

Stanislas, M., J. Kompenhans and J. Westerweel (2000). Particle image velocimetry, Kluwer Academic Pub.

Stanislas, M., K. Okamoto, C. J. Kähler and J. Westerweel (2005). "Main results of the Second International PIV Challenge." Experiments in Fluids 39(2): 170-191.

Stanislas, M., K. Okamoto, C. J. Kähler, J. Westerweel and F. Scarano (2008). "Main results of the third international PIV Challenge." Experiments in Fluids 45(1): 27-71.

Timmins, B., B. Wilson, B. Smith and P. Vlachos (2012). "A method for automatic estimation of instantaneous local uncertainty in particle image velocimetry measurements." Experiments in Fluids 53(4): 1133-1147.

Warner, S., O. and B. L. Smith (2013). "Autocorrelation-Based Estimate Of Particle Image Density For Diffraction Limited Particles." submitted to Meas. Sci. Tech.

Wereley, S. T. and L. Gui (2003). "A correlation-based central difference image correction (CDIC) method and application in a four-roll mill flow PIV measurement." Experiments in Fluids 34(1): 42-51.

Wereley, S. T. and C. D. Meinhart (2001). "Second-order accurate particle image velocimetry." Experiments in Fluids 31(3): 258-268. 
Willert, C. (1997). "Stereoscopic digital particle image velocimetry for application in wind tunnel flows." Measurement Science \& Technology 8(12): 1465-1479.

Willert, C. E. and M. Gharib (1991). "Digital particle image velocimetry." Experiments in Fluids 10(4): 181-193.

Wilson, B. M. and B. L. Smith (2013) "Uncertainty On PIV Mean And Fluctuating Velocity Due To Bias And Random Errors." Vol. 24 035302, Meas. Sci. Tech.

Wilson, B. M. and B. L. Smith (2013) "Taylor-Series And Monte-Carlo-Method Uncertainty Estimation Of The Width Of A Probability Distribution Based On Varying Bias And Random Errors," Vol. 24, 035301 Meas. Sci. Tech.

Yao, C. S. and R. J. Adrian (1984). "Orthogonal Compression and 1-D Analysis Technique for Measurement of 2-D Particle Displacements in Pulsed Laser Velocimetry." Applied Optics 23(11): 1687-1689. 


\section{Appendix B-1}

\section{Creating a New Uncertainty Surface}




\section{Appendix B-1 \\ Creating a New Uncertainty Surface}

To create a new uncertainty surface you will need the contents of the "Image and Surface Generator" folder, which contains a synthetic image generator "SIG_parallel", and the two uncertainty surface generators "DaVisSurfaceGenerator.m" and "PranaSurfaceGenerator.m".

\subsection{Step 1: Generate the Synthetic Images}

Open "SIG_parallel.m" in MATLAB. The first several lines of the code explain how to use the code. You must specify:

- If you want to code to run in parallel, and how many cores to use.

- The parameter space (diameter, density, shear, and displacement. Mind the units!) i.e.:

- $\quad$ parameters.DiaVals=[0.5 1.01 .52 .02 .53 .05 .07 .0$]$; \%(pixels)

- $\quad$ parameters.DenVals=[5 102040 80]; \%(particles/32x32 pixel region)

- $\quad$ parameters. ShrVals=[0 0.040 .080 .120 .180 .25$] ; \%$ (pixels/pixel)

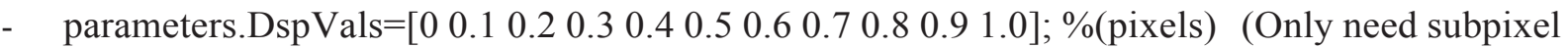
displacements between 0 to 1 )

- A shift value (uncomment a shift value based on the final grid size you will use. Do not take into account $\%$ overlap).

- If you want to add camera noise to the images. Timmins et al.[2010] showed camera noise can be neglected. (including noise will about double the space required to store the images)

- The flow type (always use selection '1', the linear velocity gradient )

- Image size (chosen to provide appropriate number of vectors for convergence $\sim 10,000$ )

- $\quad$ parameters.rows $1=128$;

- $\quad$ parameters.cols $1=160128$;

- The save location for the images. The space required depends on parameter space. Larger diameters and densities generate larger files. The parameter space above takes about 70 GB.).

Once these parameters are specified, run the code. (The parameter space above takes about 4 hours to generate using 6 cores.)

\subsection{Step 2: Process the "shifted" images in DaVis or PRANA}

Import the "shifted" images into DaVis.

The first step in your DaVis operations list needs to be "copy and reorganize" with the Operation set as "create multi-frame buffer from time series". In the operation list under the "create multi- frame buffer..." select Parameter and on the Mode dropdown select "create (n/2) image: $1+2,3+4,5+6 \ldots$...

Next specify the PIV operations and settings you wish to use when you process your actual data. However, DO NOT USE \% OVERLAP. It is okay if you want to use \% overlap when you process your data, but for the sake of keeping the vectors from the synthetic images independent, do not include overlap. Make sure that you save your operations list or record the settings for future reference. This new uncertainty surface you generate should only be used on PIV data processed using these specific settings.

Process the synthetic images.

Similar steps should be followed when using PRANA. 
Note: Generating the synthetic images takes considerable storage space. Importing them into DaVis copies and converts the png files into single image im7 files. These single im7 files are paired up, thus making another set of images. Once all is said and done you only need the combined im7 files for $\mathrm{DaVis}$ (or the single png files for Prana). For example, if you are using DaVis, once the single im7 have been combined using the "create multi-frame buffer from time series", you can delete the png and single im7 files. I would suggest keeping the combined im7 files if you plan on making more surfaces in the future using different PIV operations.

\subsection{Step 3: Generate the surface}

Going back to MATLAB, open the code titled "DaVisSurfaceGenerator.m" or

"PranaSurfaceGenerator.m", depending on what PIV software you used. There are only 4 variables you need to specify before you run the code

1. VecRow - uncomment the variable "VecRow" that corresponds to the size of your final grid resolution (you should not have used \% overlap when processing for reasons mentioned above).

2. path_name - This is the directory of the PIV vector files (vc7 for DaVis, .m for PRANA). For example: path_name='D: $\mid$ CalibrationImages $\backslash$ CreateMultiframe $\backslash$ SelfCalBalPin $\backslash^{\prime}$

3. key_loc - This is the directory of the "Key.m" file. When the synthetic images are generated a file called "Key.m" is generated that contains the parameters for each synthetic image. If you accidentally deleted the key you can generate a new one by running the first part of the SIG parallel code (using the same parameters). Place a break- point after the point where the Key is saved ( line 160) and run the code.

4. SurfName - This is the name of the new surface (i.e., 'PIVuncertainty_DaVis.mat')

This surface can be loaded into the PIVuncertainty code and used to estimate the PIV measurement uncertainty. It may be a good idea to store the new surface in a folder along with information about the PIV settings used to create it (like the operation list from $\mathrm{DaVis}$, or the experimental summary from PRANA).

\subsection{Step 4: Estimate the PIV measurement uncertainty}

See document: "Using PIVuncertaintyCode."

Questions? Contact me at scottowarner@gmail.com 
Appendix B-2

\section{Using PIVuncertaintyCode}




\section{Appendix B-2 Using PIVuncertaintyCode}

PIVuncertaintyCode.m is a code written in MATLAB used for estimating the uncertainty in PIV results using the USM. Three files are required to run the code, and they are as follows:

- PIVuncertaintyCode.m (Main Code)

- PIVuncertaintyGUI.m (GUI code)

- PIVuncertaintyGUI.fig (GUI figure)

- DiaDen.m (Sub Function)

- PIV_Stats.m (Sub Function)

- A custom uncertainty surface (ie. PIVuncertainty_DaVis.mat). If you do not have one, see document titled "Creating a New Uncertainty Surface." 
To run the code using the GUI, open PIVuncertainty.m in MATLAB and click run or press (F5). The following window will open as shown:

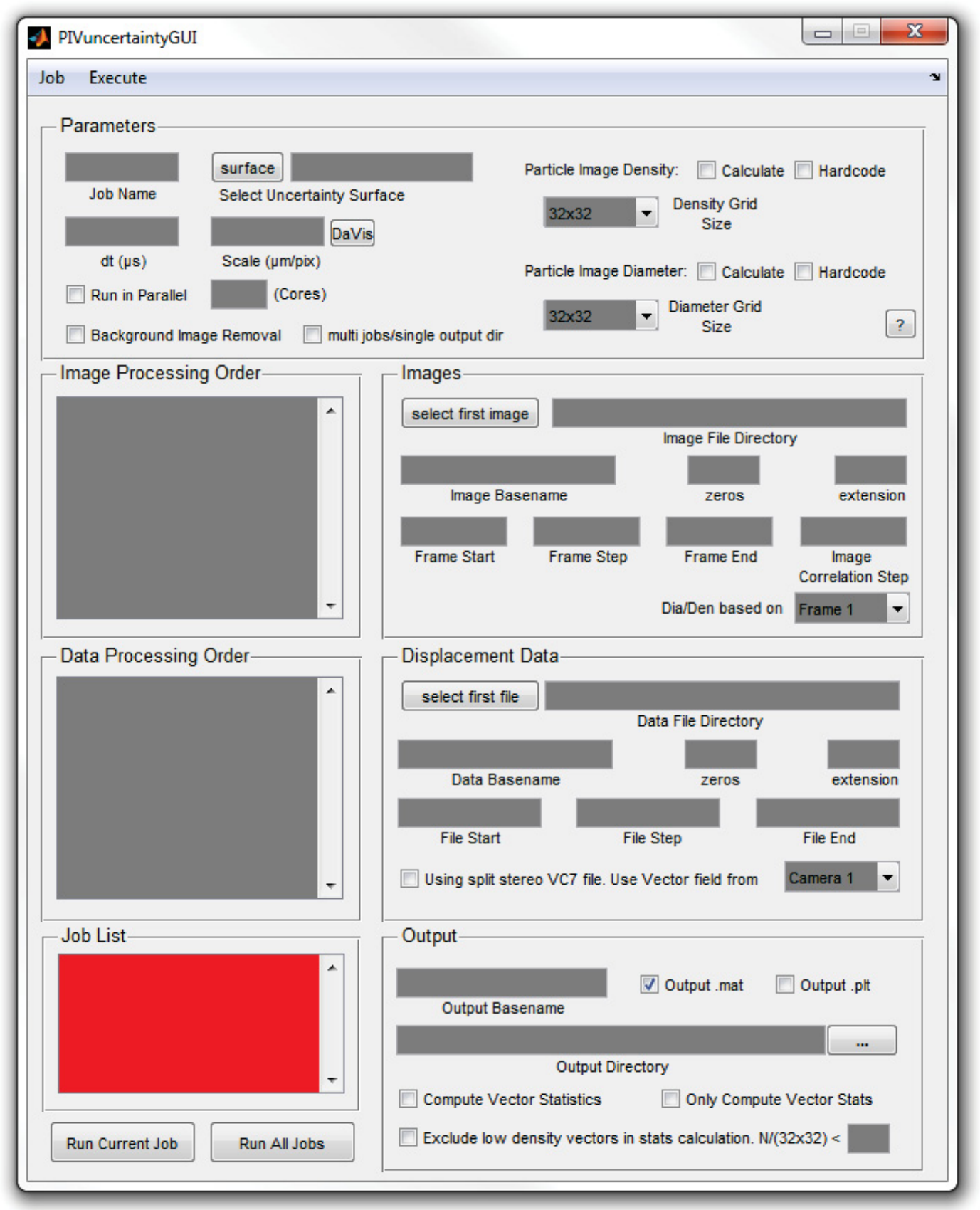

To begin a new job, select the job menu and select New. Type in a job name as directed. The new job name will appear in the Parameters section in the "Job Name" text box as well as in the Job List area.

\section{Parameters Section:}

Select which uncertainty surface to use (click the "surface" button, navigate to, and select the surface you wish to use). Note: each surface is unique for the parameters used to process your images. Before using this code, confirm that the surface you are using was generated for the way you wish to process your data.

Enter the time between images "dt $(\mu \mathrm{s})$ " and the length conversion "Scale $(\mu \mathrm{m} / \mathrm{pix})$ " in the units specified. If you are using im7 and vc7 files, you may click the "DaVis" button and auto fill these 
sections once you have loaded your im7 and vc7 files. Verify that the values entered are correct before proceeding. If the button does not work, manually enter these values. If your images and vector files are in pixel units and you wish the uncertainty values to be in pixels, you can leave these values as 1 .

Select Run in Parallel if multiple cores are available and you wish to save time. Enter the number of cores you wish to use in corresponding text box.

Select Background Image Removal to subtract a background image (this is based on the minimum value for each pixel over time). This is recommended as it removes many negative effects of noise.

If you wish to calculate the particle image density and diameter, select the grid size you wish to use (recommended $64 \times 64$ or larger). If you are not interested in calculating the Diameter or Density and have an estimate of what it should be, check the "Hardcode Particle Density" or "Hardcode Particle Diameter" box and enter in the estimate in the corresponding text box in the units shown.

\section{Loading Images:}

To load images click on the button next to the "Image File Directory" text box. Select the first image you wish to use.

The remaining text boxes in the "Images" section will auto-populate according to the first image you selected. If a images name is "B00001.im7", the "Image Basename" is "B", the number of "zeros" is "5" and the "extension" is "im7".

The "Image Correlation Step" is the difference between image pairs. For example, DaVis *.im7 files contain both images within the file so the "Image Correlation Step" is 0 . If Image 1 is paired with image 2, than the "Image Correlation Step" is 1.

"Frame Start" and "Frame End" are the first and last image in the set.

"Frame step" is the difference between the first image of any two sequential image pairs. For $\mathrm{DaV}$ is this will be set to 1 . For other images, such as when image $1 \& 2$ are a pair and $3 \& 4$ are a pair the "Frame Step" is 2.

Once the text fields in the "Images" section are filled out you should see each pair of images in the "Image Pairs" section. If the "Image Pairs" section is red, recheck the values you have entered in the "Images" section.

You can specify which image to use for the Dia/Den calculations. You can select frames 3 and 4 if you are using stereo im7 files (not currently working for other formats).

\section{Displacement Data:}

Follow the same procedure as the "Images" section. Ensure that the order of files found in the "Data Processing Order" section correspond to the image pairs found in the "Image Processing Order" section.

If you are using split stereo VC7 files, you can select the vectors from Camera 1 or Camera 2.

\section{Output Section:}

Enter a base name for the result files in the "Output Basename" field (ie 'B' for files named B00001.mat, B00002.mat, etc). Results for each image pair and PIV results will be generated (for a list of variables see the appendix of this document). Select which type of output to generate (.mat files are always created due to their use in computing the vector statistics). Click on the button to the right of the "Output .plt/.mat Directory" section to select the output location. 
Select "Compute Vector Statistics" to calculate statistics for the whole data set. These parameters include: averages, variances, and standard deviations of the $\mathrm{u}, \mathrm{v}$, and uv-magnitude velocities, the covariance, and average shear. Also provided are the uncertainty values $(95 \%)$ for the previously mention values. (For a list of variables see appendix of this document or the beginning of the PIV_Stats.m code)

If you have already ran data through the code but have not yet computed the statistics, fill out GUI information and check the box labeled "Only Compute Vector Statistics." Selecting this option will forgo re-running all of the code and skip to the PIV stats portion.

Computing vector statistics will produce a file called Ustats.mat in the location specified in the "Output Directory" text box.

\section{Job List:}

The Job list section shows all currently open jobs. Select a given job to view and change its settings.

\section{Execute Menu:}

To run a single job select the Execute menu at the top of the GUI and select "Run Job." If multiple jobs are located in the "Job List" section and you wish to run them all, select "Run All" from the "Execute" menu.

\section{Additional Features of the Job menu:}

- Save a job set up you may save it as a mat file for future use.

- Load a job you have saved.

- Copy a currently open job. A box will appear, enter the new jobs name.

- Delete a job from the job List.

A typical job may appear something like this: 


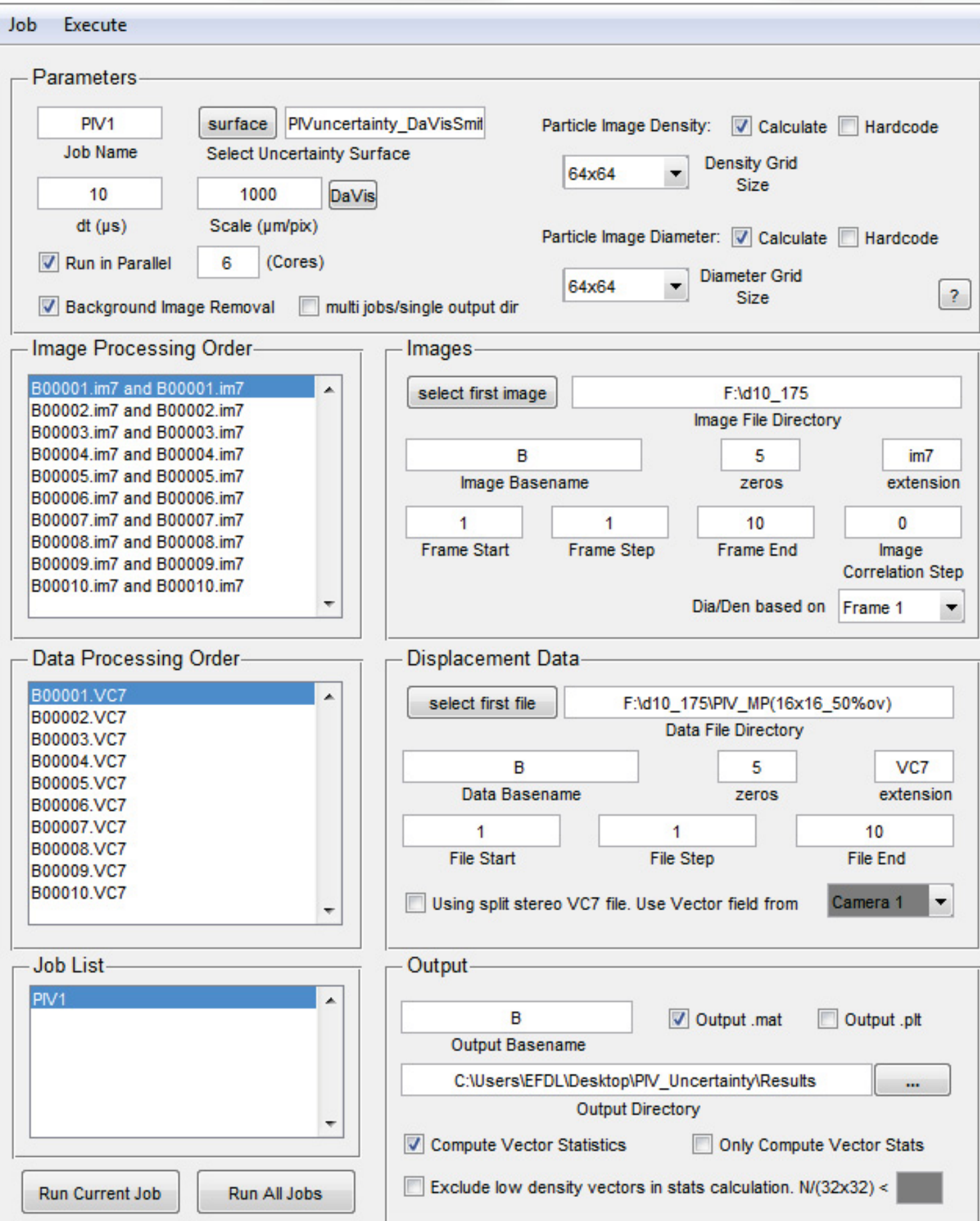




\subsection{Addendum}

\subsubsection{Input: from PIV Uncertainty (i.e., B00001.mat)}

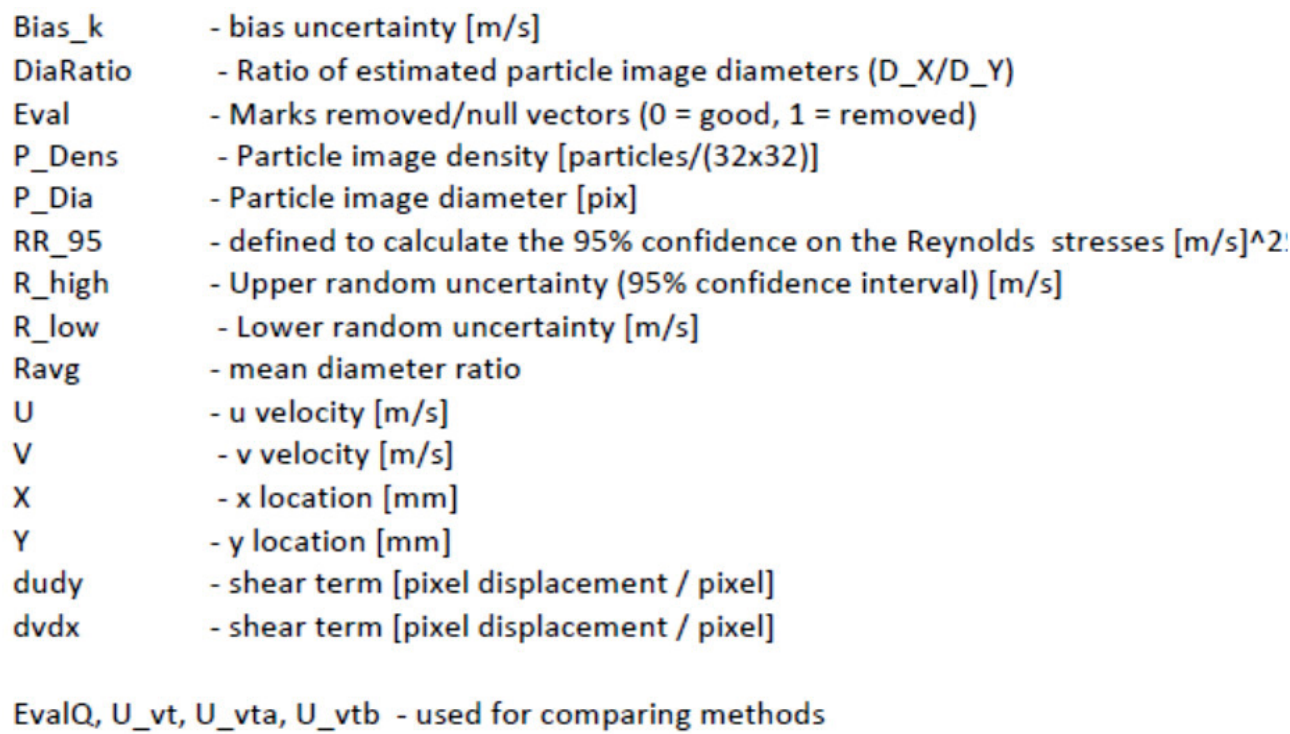

\subsubsection{Output: UStats.mat}

$\mathrm{N} \quad-$ Samples per interogation region

Location:

$\mathrm{X}-\mathrm{x}$ location $[\mathrm{mm}]$

$\mathrm{Y} \quad-\mathrm{y}$ location [mm]

Velocity

u_ave - Time averaged u velocity $[\mathrm{m} / \mathrm{s}$ ]

uv_ave - Time averaged velocity magnitude $[\mathrm{m} / \mathrm{s}]$

v_ave - Time averaged v velocity $[\mathrm{m} / \mathrm{s}$ ]

Variance/Covariance:

uut $\quad-$ u variance (u'u'bar) $[\mathrm{m} / \mathrm{s}]^{\wedge} 2$

uvt - covariance (u'v'bar) $[\mathrm{m} / \mathrm{s}]^{\wedge} 2$

vvt $\quad-v$ variance (v'v'bar) $[\mathrm{m} / \mathrm{s}]^{\wedge} 2$

uvuvt - velocity magnitude variance (uv'uv'bar) $[\mathrm{m} / \mathrm{s}]^{\wedge} 2$

Precision:

Puu - Precision Uncertainty in Variance (u'u'bar) $[\mathrm{m} / \mathrm{s}]^{\wedge} 2$

Puv - Precision Uncertainty in covariance (u'v'bar) $[\mathrm{m} / \mathrm{s}]^{\wedge} 2$

Puvuv - Precision Uncertainty in Velocity magnitude variance (uv'uv'bar) $[\mathrm{m} / \mathrm{s}]^{\wedge} 2$

Pvv - Precision Uncertainty in Variance (v'v'bar) $[\mathrm{m} / \mathrm{s}]^{\wedge} 2$

Uncertainties:

Uu $\quad$ - Time averaged u velocity uncertaity * $(\mathrm{Eq} 9)$

Uuv - Time averaged velocity magnitude uncertainty $[\mathrm{m} / \mathrm{s}]$ 


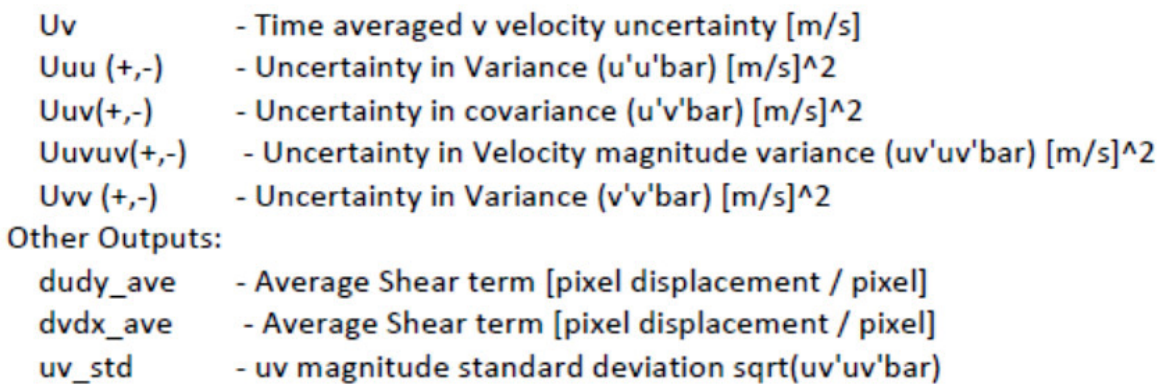


Appendix B-3

3C USM Procedure 


\section{Appendix B-3 3C USM Procedure}

This document outlines the procedure for computing 3C PIV uncertainty using the Uncertainty Surface Method (USM). In DaVis, stereo PIV is performed by first dewarping the images, processing each image pair to get two $2 \mathrm{C}$ vector fields, and combining the $2 \times 2 \mathrm{C}$ vector fields to form a single $3 \mathrm{C}$ vector field. A similar procedure is performed to compute the $3 \mathrm{C}$ uncertainty and is outlined as follows:

1. In $\mathrm{DaV}$ is, select your stereo images and go to processing.

2. Operation 1: Using the same operation list you used to process your data, change stereo cross-correlation to sequential cross-correlation. Make sure "Use image correction" is selected. For each im7 file this provides a multi-frame buffer that contains $2 \times 2 \mathrm{C}$ vector fields.

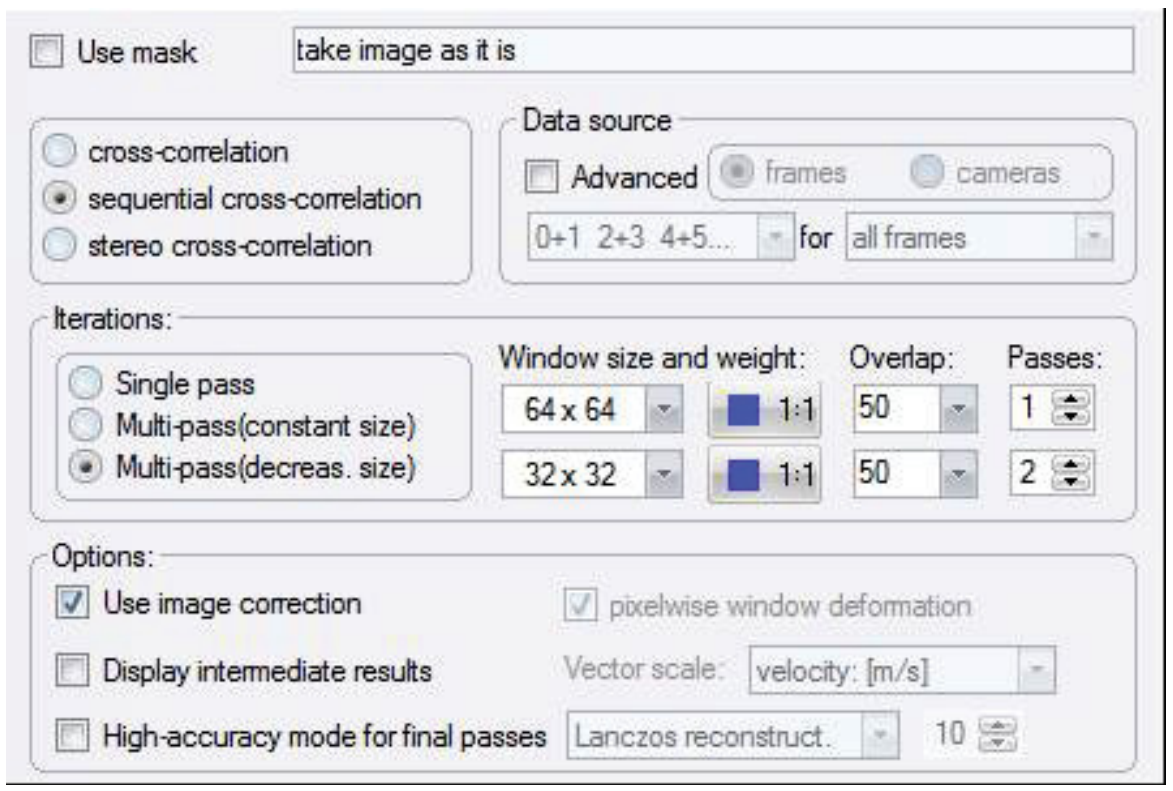

Operation 2: Separate the multi-frame buffer into time series.

\begin{tabular}{ll|} 
Operation 2: & \\
Group: & copy and reorganize frames \\
Operation: & create time series from multiframe buffer \\
\hline Storage mode: & default: \\
Storage name: & CreateTimeSeries \\
\hline
\end{tabular}

3. The stereo images also need to be dewarped (corrected) and separated into im7 files with only 2 images per file (instead of 4 images). This can be done by selecting the following operations: image correction (raw -> world) (found under image mapping group), then create time-series from multi-frame buffer (found under copy and reorganize frames), and finally create multi-frame buffer from time-series (also found under copy and reorganize frames) with Parameter Mode set to create $(n / 2)$ images: $1+2,3+4,5+6$... as shown below. 


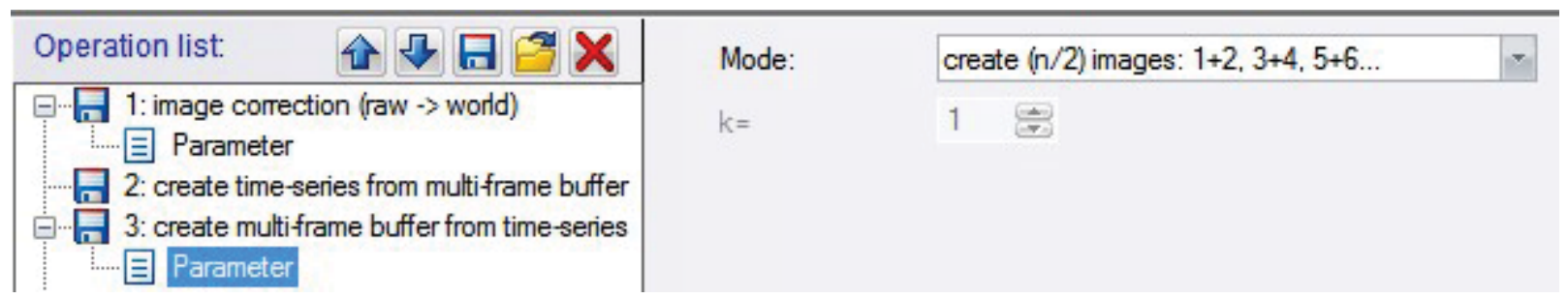

4. Process the $2 \mathrm{C} \mathrm{vc} 7$ files and dewarped $2 \mathrm{C}$ im 7 files using the USM code (see previous documents on how to make a surface and process your data using the uncertainty code). This will give you a mat file for each vc7 file. For best results when using Background Image Removal, process odd files separate from even files by using the Frame/File Start/Step boxes (i.e., Frame Start of 1 and Step of 2 will do odd and Frame Start of 2 and Step of 2 will do even files.). This will allow for background from Camera 1 and 2 to be calculated separately (instead of a single background for both cameras).

5. Run the DaVisTXT_GUI.m code. This code saves data contained in the USM mat files to txt files that can be read into $\mathrm{DaV}$ is. Each txt file contains 4 columns for $\mathrm{X}, \mathrm{Y}$, UncertParamU(x,y),

UncertParamV(x,y). There will be 4 times as many txt files as you do mat files (bias, upper random, lower random, and $\mathrm{rr} 95)$. The final output is located in the Output directory specified by the user inside a folder titled "DaVisUncertTXT".

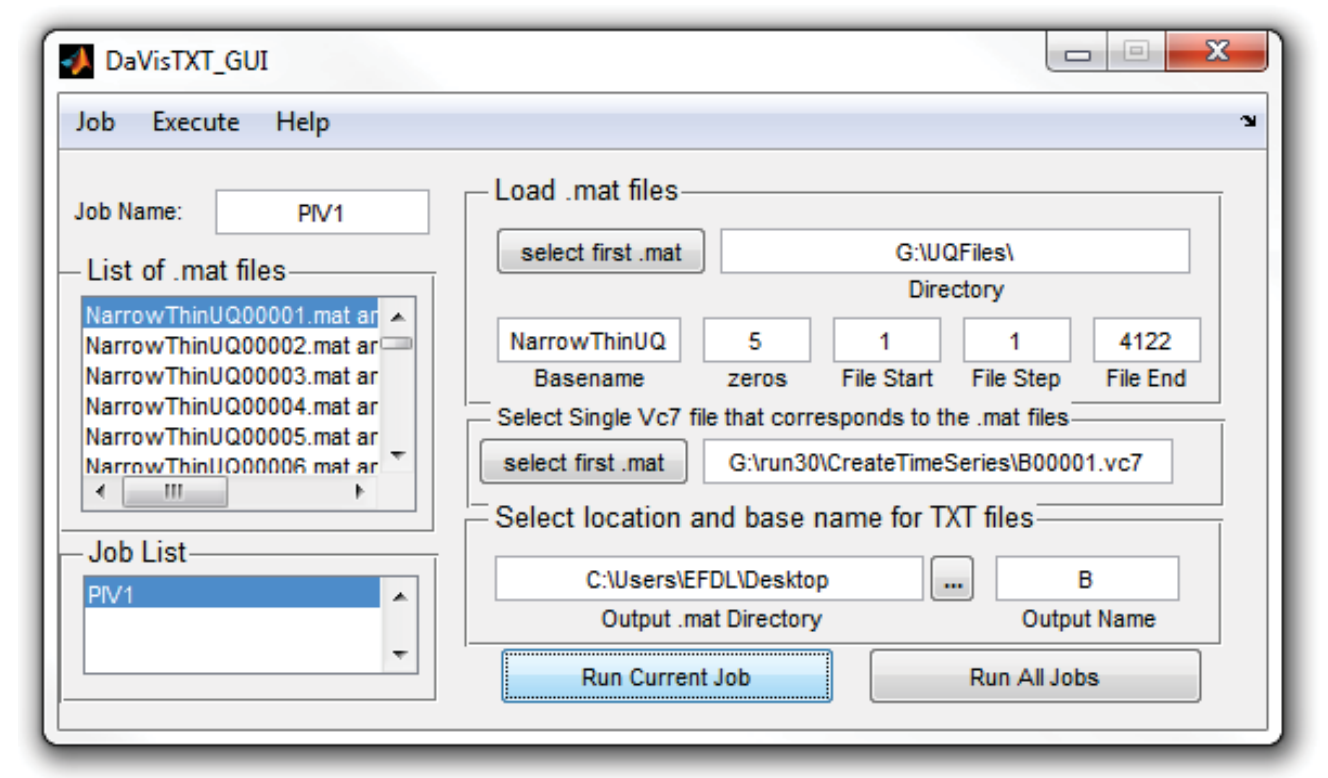

6. Load the TXT files into DaVis using the Import feature. The text files should show up as vector fields when selected. Select all the TXT files, click Add to list, and then Import Data. You must import the data into the same folder that contains you calibration information. The calibration is needed to perform the $2 \times 2 \mathrm{C}$ to $3 \mathrm{C}$ operation. 


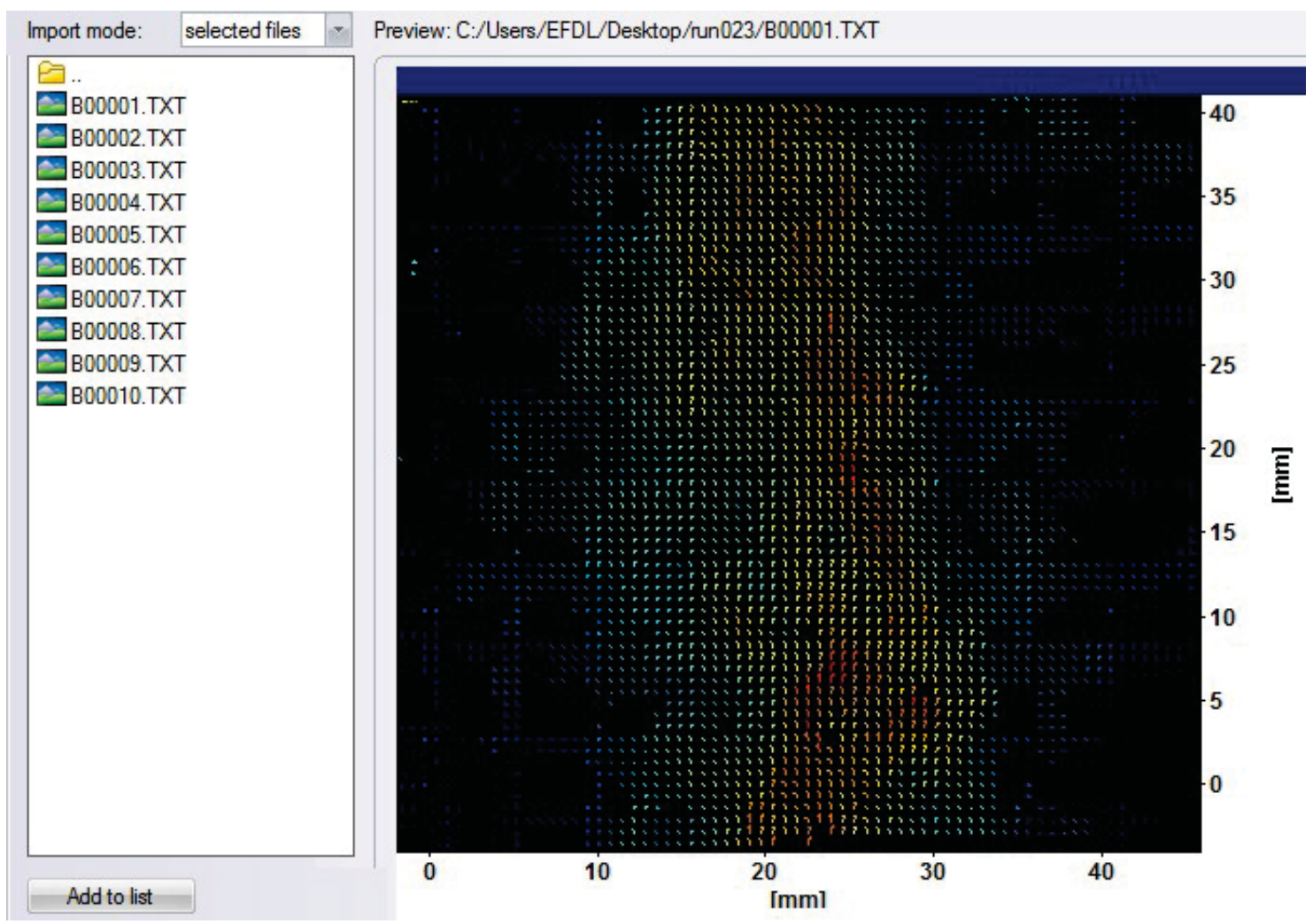

7. Once the uncertainty vector fields are imported into $\mathrm{DaV}$ is they need to be recombined from time series to multi-frame. Set the mode such that images $1+2,3+4,5+6 \ldots$ are combined. (wait to click Start Processing. Two more operations need to be set up)

\begin{tabular}{ll|}
$\begin{array}{ll}\text { Operation 3: } \\
\text { Group: }\end{array}$ & \multicolumn{1}{l}{ copy and reorganize frames } \\
\cline { 2 - 2 } Operation: & create multiframe buffer from time series \\
\hline Storage mode: & default: \\
Storage name: & CreateMultiframe \\
\hline Mode: & create $(\mathrm{n} / 2)$ images: $1+2,3+4,5+6 \ldots$ \\
\hline $\mathrm{k}=$ & 1
\end{tabular}

8. Attributes need to be added to the imported data, otherwise the $2 \mathrm{C}$ to $3 \mathrm{C}$ operation will not work correctly. The difficult part is that there is no easy way to copy attributes from the original $2 \times 2 \mathrm{C}$ velocity vc 7 files to the recently imported uncertainty vc7 files. The attributes you need to add are CameraName0, CameraName1, FrameProcessing0, and FrameProcessing1, the others seem to have no effect on the final $3 \mathrm{C}$ results. To get these attributes, click on the original $2 \times 2 \mathrm{C} \mathrm{vc7}$ files (from Step 2: operation 1) and go to processing and select the operation attributes then add/modify attributes then select Parameter. Click the dropdown menus and select the four attributes. The information will populate the right hand side (similar to what is shown below). 


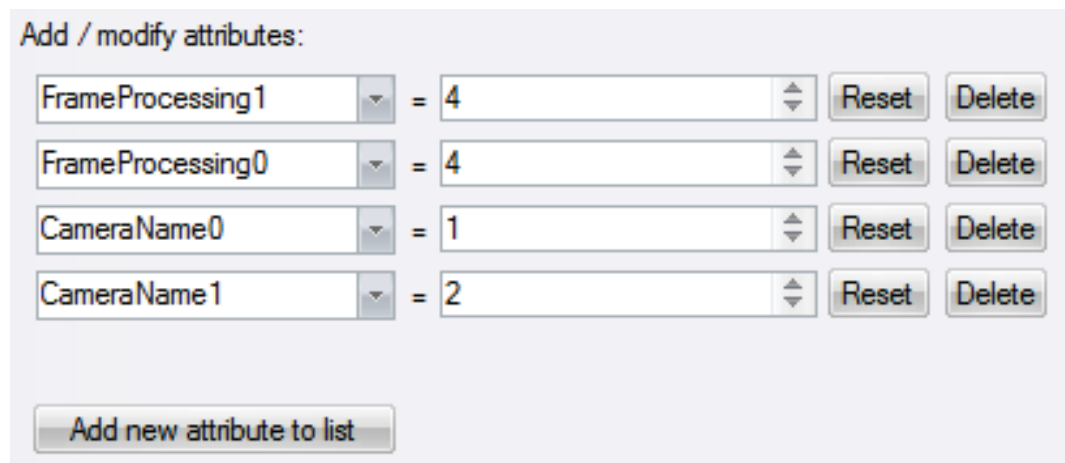

Now exit and click on the multi-frame uncertainty vc7 files and go to processing. The attributes from the previous vc7 files are still present. (If for some reason you didn't save the files from step 2, repeat step 2 (Operation 1) for a single file and use it to get the above attributes.) (wait to click Start Processing. One more operation need to be set up)

9. The final step is to convert the $2 \mathrm{C}$ vector fields into a $3 \mathrm{C}$ vector field. For the next operation select vector properties and then convert $2 x 2 D$ vector fields to $1 x$ stereo vector field.

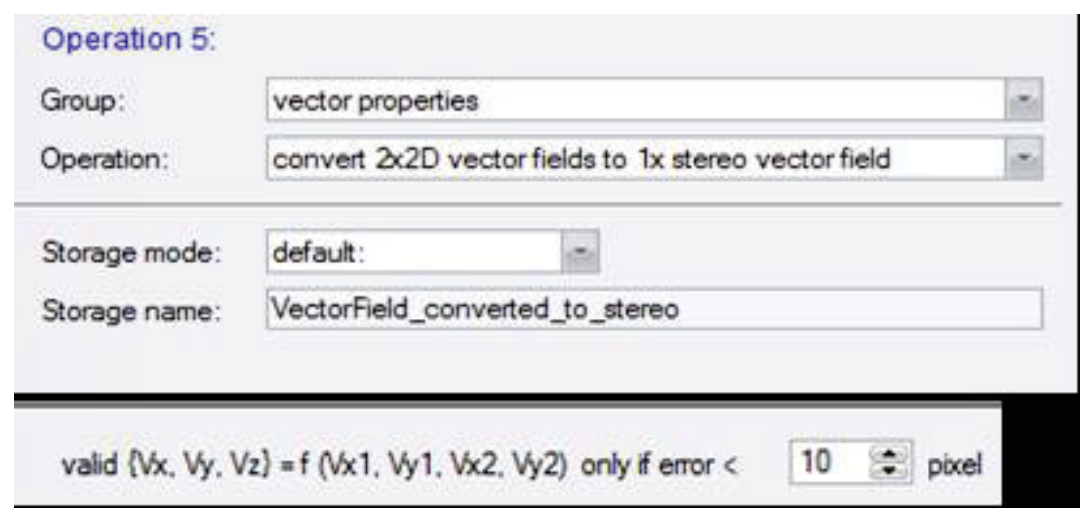

Under Parameter select an error value. Make sure to do this as the default value is 0 and will result in an error.

10. Now there should be 3 operations in the Operation list:

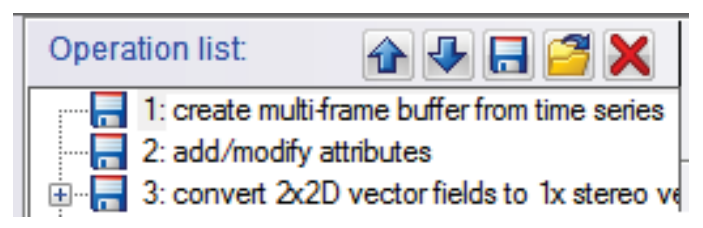

Click Start Processing to get the 3C uncertainty parameter vector fields.

11. There should now be 4 times as many uncertainty vc7 files as there are stereo vc7 files. Run the PIV_stats3C_GUI.m code. Load the stereo vc7 files and select the location of the uncertainty vc7 files.

NOTE: If you are working out of multiple folders you can run the code

PIV3C_vec2mat_GUI.m. This will process all jobs and save the 3C data as sequential .mat files. 


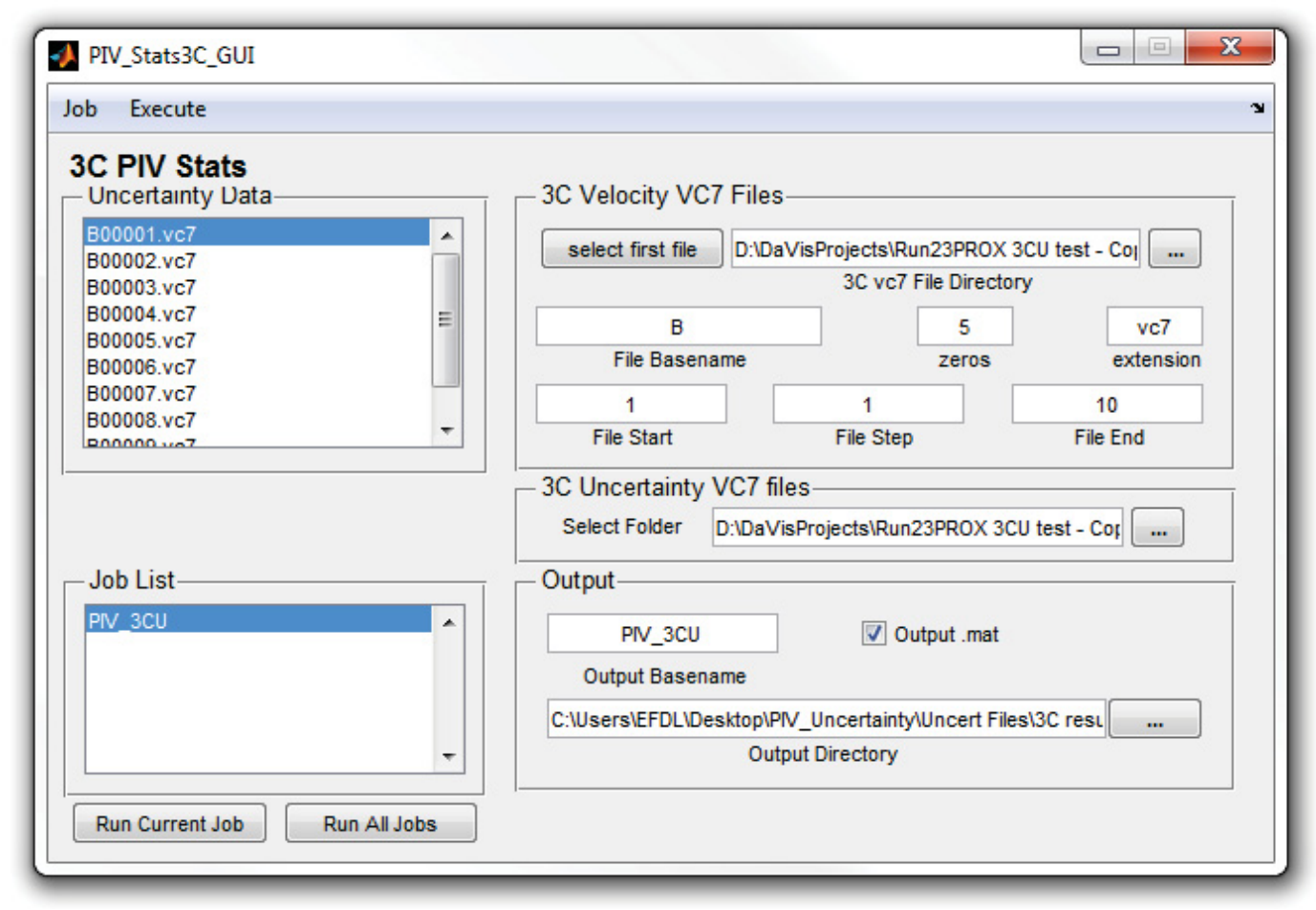

The Stats code outputs 2D matrices containing:

- $\mathrm{X}$ and $\mathrm{Y}$ positions

- Time average velocities in $\bar{u}, \bar{v}$, and $\bar{w}$ and their uncertainties

- variance and covariance terms $\overline{u^{\prime} u^{\prime}}, \overline{v^{\prime} v^{\prime}}, \overline{w^{\prime} w^{\prime}}, \overline{u^{\prime} v^{\prime}}, \overline{u^{\prime} w^{\prime}}$, and $\overline{v^{\prime} w^{\prime}}$ as well as their uncertainties

- Precision uncertainties that correspond to the varience and covarience terms listed above

This process has been tested using velocities instead of uncertainties to arrive at the same answer that DaVis provides when performing stereo PIV. The average error was less than 0.00001 pixels with a standard deviation of 0.0016 pixels.

An alternate Step 1 would be to take your already processed stereo data and convert it into $2 \times 2 \mathrm{C}$ vector fields. I checked this method by simply importing the $2 \times 2 \mathrm{C}$ velocity fields (rather than uncertainty) to try to arrive at the same $3 \mathrm{C}$ solution obtained by way of stereo PIV. When I used the velocity fields from sequential cross correlation the final $3 \mathrm{C}$ vector field matched the stereo results better than when I used the $2 \times 2 \mathrm{C}$ fields obtained by separating the stereo result. 
Operation 1:

\begin{tabular}{ll|l|} 
Group: & vector properties & - \\
Operation: & convert $1 \mathrm{x}$ stereo vector field to $2 \times 2 \mathrm{D}$ vector fields \\
\hline
\end{tabular}

Storage mode: default:

Storage name: VectorField_converted_to_2x2D 